\title{
Kinetic limit for a chain of harmonic oscillators with a point Langevin thermostat
}

\author{
Tomasz Komorowski* Stefano Olla ${ }^{\dagger}$
}

February 21, 2022

\begin{abstract}
We consider an infinite chain of coupled harmonic oscillators whose Hamiltonian dynamics is perturbed by a random exchange of momentum between particles such that total energy and momentum are conserved, modelling collision between atoms. This random exchange is rarefied in the limit, that corresponds to the hypothesis that in the macroscopic unit time only a finite number of collisions takes place (the Boltzmann-Grad limit). Furthermore, the system is in contact with a Langevin thermostat at temperature $T$ through a single particle. We prove that, after the hyperbolic space-time rescaling, the Wigner distribution, describing the energy density of phonons in space-frequency domain, converges to a positive energy density function $W(t, y, k)$ that evolves according to a linear kinetic equation, with the interface condition at $y=0$ that corresponds to reflection, transmission and absorption of phonons caused by the presence of the thermostat. The paper extends the results of [13, where a harmonic chain (with no inter-particle scattering) in contact with a Langevin thermostat has been considered.
\end{abstract}

\section{Introduction}

The mathematical analysis of macroscopic energy transport in anharmonic chain of oscillators constitutes a very hard mathematical problem, see [21]. One approach to it is to replace the non-linearity by a stochastic exchange of momentum between nearest neighbor particles in such a way that the total kinetic energy and momentum are conserved. This stochastic exchange can be modeled in

\footnotetext{
*Institute of Mathematics, Polish Academy Of Sciences, ul. Śniadeckich 8, 00-956 Warsaw, Poland, e-mail: komorow@hektor .umcs. lublin.pl

${ }^{\dagger}$ CEREMADE, Université de Paris Dauphine, PSL Research University, Institut Universitaire de France, GSSI, L'Aquila, e-mail: olla@ceremade.dauphine.fr
} 
various ways: e.g. for each couple of nearest neighbor particles the exchange of their momenta can occur independently at an exponential time (which models their elastic collision). Otherwise, for each triple of consecutive particles, exchange of momenta can be performed in a continuous, diffusive fashion, so that its energy and momentum are preserved. In the present article we adopt the latter choice, see Section 2.3.1 below for a detailed description of the dynamics. In fact, with no significant changes, all our results can be extended to some other stochastic noises that are both local in the spatial variable and of independent increments in time, such as e.g. the Poisson random exchange mentioned above (see [5, Section 5.1.3]).

A small parameter $\epsilon>0$, describing the ratio between macroscopic and microscopic space-time units, is introduced. The intensity of the noise is adjusted so that in a (macroscopic) finite interval of time, there is only a finite amount of momentum exchanged by the stochastic mechanism. In terms of the random exchanges, it means that, on average, each particle undergoes only a finite number of stochastic collisions in a finite time. Letting $\epsilon \rightarrow 0$ corresponds therefore to taking the kinetic limit for the system, since in the case of a chain with no microscopic boundary present the energy density evolution is described by a linear kinetic equation, see [4.

The Wigner distribution is a useful tool to localize in space the energy per frequency mode. In the absence of the thermostat, it is proven in [4, that as $\epsilon \rightarrow 0$, the Wigner distribution converges to the solution of the kinetic transport equation

$\partial_{t} W(t, y, k)+\bar{\omega}^{\prime}(k) \partial_{y} W(t, y, k)=2 \gamma_{0} \int_{\mathbb{T}} R\left(k, k^{\prime}\right)\left(W\left(t, y, k^{\prime}\right)-W(t, y, k)\right) d k,(t, y, k) \in[0,+\infty) \times \mathbb{T} \times \mathbb{R}$,

with an explicitly given scattering kernel $R\left(k, k^{\prime}\right) \geq 0$. It is symmetric and the total scattering kernel behaves as

$$
R(k):=\int_{\mathbb{T}} R\left(k, k^{\prime}\right) d k^{\prime} \sim|k|^{2} \quad \text { for }|k| \ll 1 .
$$

Here $\mathbb{T}$ is the unit torus, which is the interval $[-1 / 2,1 / 2]$, with identified endpoints. Furthermore $\gamma_{0}>0$ is the scattering rate for the microscopic chain (see (2.8) below) and $\bar{\omega}(k)=\omega(k) / 2 \pi$, where $\omega(k)$ is the dispersion relation of the chain (see definition (2.11) $)$.

In the present paper we are interested in the macroscopic effects of a heat bath at temperature $T$, modeled by a Langevin dynamics, applied to one particle, say the one labelled 0 , with a coupling strength $\gamma_{1}>0$ (see (2.8) below for a detailed description). Unlike the conservative stochastic dynamics acting on the bulk, the action of the heat bath is not rescaled with $\epsilon$. In the macroscopic coordinates it is represented by large terms (a deterministic and stochastic ones of orders $\epsilon^{-1}$ and $\epsilon^{-1 / 2}$ respectively), see (2.15) below. Therefore the presence of a thermostat can be considered as a 
singular perturbation of the dynamics of the closed system. The effect in the limit, as $\epsilon \rightarrow 0$, is to introduce the following interface conditions at $y=0$ on (1.1):

$$
\begin{array}{ll}
W\left(t, 0^{+}, k\right)=p_{-}(k) W\left(t, 0^{+},-k\right)+p_{+}(k) W\left(t, 0^{-}, k\right)+g(k) T, & \text { for } 0<k \leq 1 / 2, \\
W\left(t, 0^{-}, k\right)=p_{-}(k) W\left(t, 0^{-},-k\right)+p_{+}(k) W\left(t, 0^{+}, k\right)+g(k) T, & \text { for }-1 / 2<k<0 .
\end{array}
$$

Interpreting $W(t, y, k)$ as the density of the energy of the phonons of mode $k$ at time $t$ and position $y$, then $p_{+}(k), p_{-}(k)$ and $\mathrm{g}(k)$ are respectively the probabilities for transmission, reflection and absorption of a phonon of mode $k$ when it crosses $y=0$, while $g(k) T$ is the rate of creation of a phonon of that mode. These probabilities are functions of $k$ and depend only on the dispersion relation $\omega(\cdot)$ and intensity $\gamma_{1}$ of the thermostat (cf. (2.29)). They are properly normalized, i.e. $p_{+}(k)+p_{-}(k)+g(k)=1$, so that $W(t, y, k)=T$ is a stationary solution (thermal equilibrium).

This result was recently proven in the absence of the conservative noise in the bulk (i.e. $\gamma_{0}=0$ in (1.1)), see [13]. Then, the resulting dynamics outside the interface, given by (1.1), reduces itself to pure transport as $\gamma_{0}=0$. Obviously, the coefficients appearing in the interface conditions (1.3) do not depend on the presence of the bulk noise.

The goal of the present paper is to extend the result of [13] to the case when the inter-particle noise is present, i.e. $\gamma_{0}>0$, see Theorem 2.9 below for the precise formulation of our main result. We emphasize that in the situation when $\gamma_{0}=0$, both the equations for the microscopic and macroscopic dynamics, given below by (2.15) and (1.1) respectively, can be solved explicitly, in terms of the initial condition, and this fact has been extensively used in the proof in [13]. The argument can be extended to the dynamics where only the damping terms of the noise are present, i.e. with no noise input both from the inter-particle scattering and the thermostat, see (3.1). The equation for the macroscopic limit of the respective Wigner distribution $W^{\text {un }}(t, y, k)$ reads (cf (2.34) below), see Theorem 5.6 below,

$$
\partial_{t} W^{\mathrm{un}}(t, y, k)+\bar{\omega}^{\prime}(k) \partial_{y} W^{\mathrm{un}}(t, y, k)=-2 \gamma_{0} R(k) W^{\mathrm{un}}(t, y, k), \quad y \neq 0
$$

with the boundary conditions as in (1.3). In the next step we add the stochastic part corresponding to the inter-particle scattering, which corresponds to $T=0$ for the thermostat, see equation (4.2) formulated for the respective wave function. Next, we use the previously described dynamics to represent the solution of the equation with the help of the Duhamel formula, see (5.8). The corresponding representation for the Wigner distribution is given in (5.12). Having already established the macroscopic limit for the dynamics with no stochastic noise, we can use the Duhamel representation to identify the kinetic limit of the noisy microscopic dynamics when the thermostat 
temperature $T=0$, see Theorem [5.5. The extension to the case when the temperature $T>0$ is possible by another application of the Duhamel formula, see Section 6. We end this brief description of our argument with the remark that our method relies quite substantially on the fact that the noise in the bulk has independent increments in time, so we can treat the respective stochastic terms as a perturbation of the microscopic dynamics (involving the Itô correction terms) whose limit can be found by explicit calculations.

Concerning the derivation of the kinetic limit from the wave equation in the presence of boundaries, or sources, as far as we know, there exist only a few results dealing with the subject. We mention here the papers [17, 18, 19], where the high-frequency asymptotics of the solutions of the Cauchy problem for either the Schrödinger, or scalar wave equation with discontinuous coefficients, corresponding to a two component medium, has been considered and the semiclassical limit of the probability density (in the case of the Schrödinger) and energy density (for the wave equation) are identified. In Benamou et al [7] the Wigner distribution method is used to study the geometrical optics limit of the Helmholtz equation with source terms. In the aforementioned results the coefficients of the equation are not random. The kinetic limit has been derived from the random Schrödinger equation in the whole space only when no sources, or boundaries are present, see [20, 11, 9] for time independent potential and [1, 2, 3, 12], where the time dependent potentials have been treated. The derivation from the wave equation has been shown in [4, 15, 8]. The wave equation with no randomness in the bulk and an interface, given by a point Langevin thermostat, has been considered in the aforementioned paper [13]. To the best of our knowledge the present paper contains the first derivation of the kinetic limit from the wave equation in the presence of both randomness in the bulk and an interface. The long time, large space scale asymptotics of the kinetic limit obtained in the present article has been discussed in [6, 14].

Concerning the organization of the paper, Section 2 is devoted to preliminaries and the formulation of the main result, see Theorem 2.9, Among things discussed there is the rigorous definition of a solution of a kinetic equation (1.1) with the interface condition (1.3), see Sections 2.6 .3 and 2.6.4. Section 3 deals with the basic properties of the microscopic dynamics obtained by removal of stochastic noises, both between the particles of the chain and the thermostat. This dynamics is an auxiliary tool for the mild formulation of the microscopic dynamics corresponding to the chain with inter-particle scattering and thermostat. We discuss first the case when thermostat is set at $T=0$, see Section 4, In this section we obtain also basic estimates for the microscopic Wigner distributions, see Proposition 4.1, that follow from the energy balance equation established in (4.23). 
A similar result is also formulated for the auxiliary dynamics with no stochastic noise in Section 5.1 , In Section 5.3 we formulate the result concerning the kinetic limit for this dynamics, see Theorem 5.6. Its proof is quite analogous to the argument of [13] and is given in Appendix B, Section 5.5] is essentially devoted to the proof of the main result (Theorem 2.9) for the case $T=0$ and the proof for $T>0$ is presented in Section 6. Some properties of the dynamics corresponding to the macroscopic kinetic limit are proven in Appendix $\mathrm{A}$. Section $\mathrm{C}$ of the appendix is devoted to the proof of some properties of the interface coefficients appearing in the limit.

\section{Acknowledgements}

T.K. acknowledges the support of the National Science Centre: NCN grant DEC-2016/23/B/ST1/00492. S.O. acknowledges the ANR-15-CE40-0020-01 LSD grant of the French National Research Agency.

\section{Preliminaries and statement of the main results}

\subsection{Basic notation}

We shall use the following notation: let $\mathbb{R}_{*}:=\mathbb{R} \backslash\{0\}, \mathbb{R}_{+}:=(0,+\infty), \mathbb{R}_{-}:=(-\infty, 0)$ and likewise $\mathbb{T}_{*}:=\mathbb{T} \backslash\{0\}, \mathbb{T}_{+}:=(0,1 / 2), \mathbb{T}_{-}:=(-1 / 2,0)$. Throughout the paper we use the short hand notation

$$
\mathfrak{s}(k):=\sin (\pi k) \quad \mathfrak{c}(k):=\cos (\pi k), \quad k \in \mathbb{T} .
$$

Let $e_{x}(k):=\exp \{2 \pi i x k\}$ for $x$ belonging to the set of integers $\mathbb{Z}$. The Fourier series corresponding to a complex valued sequence $\left(f_{x}\right)_{x \in \mathbb{Z}}$ belonging to $\ell_{2}$ - the Hilbert space of square integrable sequences of complex numbers - is given by

$$
\hat{f}(k)=\sum_{x \in \mathbb{Z}} f_{x} e_{x}^{\star}(k), \quad k \in \mathbb{T} .
$$

Here $z^{\star}$ is the complex conjugate of $z \in \mathbb{C}$. By the Parseval identity $\hat{f} \in L^{2}(\mathbb{T})$ - the space of complex valued, square integrable functions - and $\|\hat{f}\|_{L^{2}(\mathbb{T})}=\|f\|_{\ell_{2}}$.

Given $\epsilon>0$ we let $\mathbb{Z}_{\epsilon}:=(\epsilon / 2) \mathbb{Z}$ and $\mathbb{T}_{\epsilon}:=(2 / \epsilon) \mathbb{T}$. Let $\ell_{2, \epsilon}$ be the space made of all complex valued square integrable sequences $\left(f_{y}\right)_{y \in \mathbb{Z}_{\epsilon}}$ equipped with the norm

$$
\|f\|_{\ell_{2, \epsilon}}:=\left\{\frac{\epsilon}{2} \sum_{y \in \mathbb{Z}_{\epsilon}}\left|f_{y}\right|^{2}\right\}^{1 / 2} .
$$


Let

$$
\hat{f}(\eta)=\frac{\epsilon}{2} \sum_{y \in \mathbb{Z}_{\epsilon}} f_{y} e_{y}^{\star}(\eta), \quad \eta \in \mathbb{T}_{\epsilon} .
$$

The Parseval identity takes then the form $\|\hat{f}\|_{L^{2}\left(\mathbb{T}_{\epsilon}\right)}=\|f\|_{\ell_{2, \epsilon}}$.

For any non-negative functions $f, g$ acting on a set $A$ the notation $f \preceq g$ means that there exists a constant $C>0$ such that $f(a) \leq C g(a)$ for $a \in A$. We shall write $f \approx g$ if $f \preceq g$ and $g \preceq f$.

Given a function $f: \overline{\mathbb{R}}_{+} \rightarrow \mathbb{C}$ satisfying $|f(t)| \leq C e^{M t}$, for fome $C, M>0$ we denote by $\tilde{f}(\lambda)$ its Laplace transform

$$
\tilde{f}(\lambda)=\int_{0}^{+\infty} e^{-\lambda t} f(t) d t, \quad \operatorname{Re} \lambda>M
$$

\subsection{Some function spaces}

For a given $G \in \mathcal{S}(\mathbb{R} \times \mathbb{T})$ - the class of Schwartz functions on $\mathbb{R} \times \mathbb{T}$ - we let

$$
\widehat{G}(\eta, k)=\int_{\mathbb{R}} e_{y}^{\star}(\eta) G(y, k) d y
$$

be its Fourier transform in the first variable. Let

$$
\mathcal{A}_{c}:=\left[G: \hat{G} \in C_{c}^{\infty}(\mathbb{R} \times \mathbb{T})\right]
$$

Let $\mathcal{A}$ be the Banach space obtained by the completion of $\mathcal{A}_{c}$ in the norm

$$
\|G\|_{\mathcal{A}}:=\int_{\mathbb{R}} \sup _{k \in \mathbb{T}}|\widehat{G}(\eta, k)| d \eta, \quad G \in \mathcal{A}_{c}
$$

Space $\mathcal{A}^{\prime}$ - the dual to $\mathcal{A}$ - consists of all distributions $G \in \mathcal{S}^{\prime}(\mathbb{R} \times \mathbb{T})$ of the form

$$
\langle G, F\rangle=\int_{\mathbb{R} \times \mathbb{T}} \widehat{G}^{\star}(\eta, k) \widehat{F}(\eta, k) d \eta d k, \quad F \in \mathcal{A}
$$

for some measurable function $\widehat{G}: \mathbb{R} \times \mathbb{T} \rightarrow \mathbb{C}$, equipped with the norm

$$
\|G\|_{\mathcal{A}^{\prime}}=\sup _{\eta \in \mathbb{R}} \int_{\mathbb{T}}|\widehat{G}(\eta, k)| d k<+\infty .
$$

We shall also consider the spaces $\mathcal{L}_{2, \epsilon}:=\ell_{2, \epsilon} \otimes L^{2}(\mathbb{T})$. The respective norms of $G: \mathbb{Z}_{\epsilon} \times \mathbb{T} \rightarrow \mathbb{C}$ and $\widehat{G}: \mathbb{T}_{\epsilon} \times \mathbb{T} \rightarrow \mathbb{C}$ are given by

$$
\|G\|_{\mathcal{L}_{2, \epsilon}}:=\left\{\frac{\epsilon}{2} \sum_{y \in \mathbb{Z}_{\epsilon}}\left\|G_{y}\right\|_{L^{2}(\mathbb{T})}^{2}\right\}^{1 / 2}=\|\widehat{G}\|_{L^{2}\left(\mathbb{T}_{\epsilon} \times \mathbb{T}\right)} .
$$




\subsection{Infinite system of interacting harmonic oscillators}

\subsubsection{Hamiltonian dynamics with momentum and energy conserving noise in contact with a Langevin thermostat}

We consider a stochastically perturbed chain of harmonic oscillators with a Langevin thermostat at a fixed temperature $T \geq 0$ at $x=0$. Its dynamics is described by the system of Itô stochastic differential equations

$$
\begin{aligned}
& d \mathfrak{q}_{x}(t)=\mathfrak{p}_{x}(t) d t \\
& d \mathfrak{p}_{x}(t)=\left[-(\alpha \star \mathfrak{q}(t))_{x}-\frac{\epsilon \gamma_{0}}{2}(\theta \star \mathfrak{p}(t))_{x}\right] d t+\sqrt{\epsilon \gamma_{0}} \sum_{k=-1,0,1}\left(Y_{x+k} \mathfrak{p}_{x}(t)\right) d w_{x+k}(t) \\
& +\left(-\gamma_{1} \mathfrak{p}_{0}(t) d t+\sqrt{2 \gamma_{1} T} d w(t)\right) \delta_{0, x}, \quad x \in \mathbb{Z} .
\end{aligned}
$$

Here

$$
Y_{x}:=\left(\mathfrak{p}_{x}-\mathfrak{p}_{x+1}\right) \partial_{\mathfrak{p}_{x-1}}+\left(\mathfrak{p}_{x+1}-\mathfrak{p}_{x-1}\right) \partial_{\mathfrak{p}_{x}}+\left(\mathfrak{p}_{x-1}-\mathfrak{p}_{x}\right) \partial_{\mathfrak{p}_{x+1}}
$$

and $\left(w_{x}(t)\right)_{t \geq 0}, x \in \mathbb{Z}$ with $(w(t))_{t \geq 0}$, are i.i.d. one dimensional, real valued, non-anticipative standard Brownian motions, over some filtered probability space $\left(\Sigma, \mathcal{F},\left(\mathcal{F}_{t}\right)_{t \geq 0}, \mathbb{P}\right)$. In addition,

$$
\theta_{x}=\Delta \theta_{x}^{(0)}:=\theta_{x+1}^{(0)}+\theta_{x-1}^{(0)}-2 \theta_{x}^{(0)}
$$

with

$$
\theta_{x}^{(0)}=\left\{\begin{aligned}
-4, & x=0 \\
-1, & x= \pm 1 \\
0, & \text { if otherwise. }
\end{aligned}\right.
$$

A simple calculation shows that

$$
\hat{\theta}(k)=8 \mathfrak{s}^{2}(k)\left(1+2 \mathfrak{c}^{2}(k)\right), \quad k \in \mathbb{T} .
$$

Parameters $\epsilon \gamma_{0}>0, \gamma_{1}$ describe the strength of the inter-particle and thermostat noises, respectively. In what follows we shall assume that $\epsilon>0$ is small, that corresponds to the weak noise hypothesis that results in atoms suffering finitely many "collisions" in a macroscopic unit of time (the Boltzmann-Grad limit).

Since the vector field $Y_{x}$ is orthogonal both to a sphere $\mathfrak{p}_{x-1}^{2}+\mathfrak{p}_{x}^{2}+\mathfrak{p}_{x+1}^{2} \equiv$ const and plane $\mathfrak{p}_{x-1}+\mathfrak{p}_{x}+\mathfrak{p}_{x+1} \equiv$ const, the inter-particle noise conserves locally the kinetic energy and momentum.

Concerning the Hamiltonian part of the dynamics, we assume (cf [4]) that the coupling constants $\left(\alpha_{x}\right)_{x \in \mathbb{Z}}$ satisfy the following: 
a1) they are real valued and there exists $C>0$ such that $\left|\alpha_{x}\right| \leq C e^{-|x| / C}$ for all $x \in \mathbb{Z}$,

a2) $\hat{\alpha}(k)$ is also real valued and $\hat{\alpha}(k)>0$ for $k \neq 0$ and in case $\hat{\alpha}(0)=0$ we have $\hat{\alpha}^{\prime \prime}(0)>0$.

The above conditions imply that both functions $x \mapsto \alpha_{x}$ and $k \mapsto \hat{\alpha}(k)$ are even. In addition, $\hat{\alpha} \in C^{\infty}(\mathbb{T})$ and in case $\hat{\alpha}(0)=0$ we have $\hat{\alpha}(k)=k^{2} \phi\left(k^{2}\right)$ for some strictly positive $\phi \in C^{\infty}(\mathbb{T})$. The dispersion relation $\omega: \mathbb{T} \rightarrow \overline{\mathbb{R}}_{+}$, given by

$$
\omega(k):=\sqrt{\hat{\alpha}(k)}
$$

is even. Throughout the paper it is assumed to be unimodal, i.e. increasing on $[0,1 / 2]$ and then, in consequence, decreasing on $[-1 / 2,0]$. Its unique minimum and maximum, attained at $k=0$, $k=1 / 2$, respectively are denoted by $\omega_{\min } \geq 0$ and $\omega_{\max }$, correspondingly. We denote the two branches of its inverse by $\omega_{ \pm}:\left[\omega_{\min }, \omega_{\max }\right] \rightarrow \overline{\mathbb{T}}_{ \pm}$.

\subsubsection{Initial data}

We assume that the initial data is random and, given $\epsilon>0$, distributed according to probabilistic measure $\mu_{\epsilon}$ and

$$
\mathcal{E}_{*}:=\sup _{\epsilon \in(0,1]} \epsilon \sum_{x \in \mathbb{Z}}\left\langle\mathfrak{e}_{x}\right\rangle_{\mu_{\epsilon}}<+\infty .
$$

Here $\langle\cdot\rangle_{\mu_{\epsilon}}$ is the expectation with respect to $\mu_{\epsilon}$ and the microscopic energy density

$$
\mathfrak{e}_{x}:=\frac{1}{2}\left(\mathfrak{p}_{x}^{2}+\sum_{x^{\prime} \in \mathbb{Z}} \alpha_{x-x^{\prime}} \mathfrak{q}_{x} \mathfrak{q}_{x^{\prime}}\right)
$$

The assumption (2.12) ensures that the macroscopic energy density of the chain is finite.

\subsection{Kinetic scaling of the wave-function}

To observe macroscopic effects of the inter-particle scattering consider time of the order $t / \epsilon$. It is also convenient to introduce the wave function that, adjusted to the macroscopic time, is given by

$$
\psi^{(\epsilon)}(t):=\tilde{\omega} \star \mathfrak{q}\left(\frac{t}{\epsilon}\right)+i \mathfrak{p}\left(\frac{t}{\epsilon}\right)
$$

where $(\mathfrak{p}(t), \mathfrak{q}(t))$ satisfies (2.8) and $\left(\tilde{\omega}_{x}\right)_{x \in \mathbb{Z}}$ are the Fourier coefficients of the dispersion relation, see (2.11). We shall consider the Fourier transform of the wave function, given by

$$
\hat{\psi}^{(\epsilon)}(t, k)=\omega(k) \hat{\mathfrak{q}}^{(\epsilon)}(t, k)+i \hat{\mathfrak{p}}^{(\epsilon)}(t, k)
$$


Here $\hat{\mathfrak{q}}^{(\epsilon)}(t), \hat{\mathfrak{p}}^{(\epsilon)}(t)$ are given by the Fourier series for $\mathfrak{q}_{x}^{(\epsilon)}(t):=\mathfrak{q}_{x}(t / \epsilon)$ and $\mathfrak{p}_{x}^{(\epsilon)}(t):=\mathfrak{p}_{x}(t / \epsilon), x \in \mathbb{Z}$, respectively. They satisfy

$$
\begin{aligned}
& d \hat{\psi}^{(\epsilon)}(t, k)=\left\{-\frac{i}{\epsilon} \omega(k) \hat{\psi}^{(\epsilon)}(t, k)-2 i \gamma_{0} R(k) \hat{\mathfrak{p}}^{(\epsilon)}(t, k)-\frac{i \gamma_{1}}{\epsilon} \int_{\mathbb{T}} \hat{\mathfrak{p}}^{(\epsilon)}\left(t, k^{\prime}\right) d k^{\prime}\right\} d t \\
& -2 \sqrt{\gamma_{0}} \int_{\mathbb{T}} r\left(k, k^{\prime}\right) \hat{\mathfrak{p}}^{(\epsilon)}\left(t, k-k^{\prime}\right) B\left(d t, d k^{\prime}\right)+i \sqrt{\frac{2 \gamma_{1} T}{\epsilon}} d w(t), \\
& \hat{\psi}^{(\epsilon)}(0)=\hat{\psi}
\end{aligned}
$$

where

$$
\begin{aligned}
& \hat{\mathfrak{p}}^{(\epsilon)}(t, k):=\frac{1}{2 i}\left[\hat{\psi}^{(\epsilon)}(t, k)-\left(\hat{\psi}^{(\epsilon)}\right)^{\star}(t,-k)\right], \\
& r\left(k, k^{\prime}\right):=4 \mathfrak{s}(k) \mathfrak{s}\left(k-k^{\prime}\right) \mathfrak{s}\left(2 k-k^{\prime}\right) \quad k, k^{\prime} \in \mathbb{T}, \\
& R(k):=\int_{\mathbb{T}} r^{2}\left(k, k^{\prime}\right) d k^{\prime}=\mathfrak{s}^{2}(2 k)+2 \mathfrak{s}^{2}(k)=\frac{\hat{\theta}(k)}{4} .
\end{aligned}
$$

Here $B(t, d k)=\sum_{x \in \mathbb{Z}} w_{x}(t) e_{x}(k) d k$ is a cylindrical Wiener process on $L^{2}(\mathbb{T})$, i.e.

$$
\mathbb{E}\left[B(d t, d k) B^{\star}\left(d s, d k^{\prime}\right)\right]=\delta\left(k-k^{\prime}\right) \delta(t-s) d t d s d k d k^{\prime}
$$

\subsection{Energy density - Wigner function}

One can easily check that

$$
\left\|\hat{\psi}^{(\epsilon)}(t)\right\|_{L^{2}(\mathbb{T})}^{2}=\left\|\psi^{(\epsilon)}(t)\right\|_{\ell^{2}}^{2}=\sum_{x \in \mathbb{Z}}\left(\mathfrak{p}_{x}^{(\epsilon)}(t)\right)^{2}+\sum_{x, x^{\prime} \in \mathbb{Z}} \alpha_{x-x^{\prime}} \mathfrak{q}_{x}^{(\epsilon)}(t) \mathfrak{q}_{x^{\prime}}^{(\epsilon)}(t) .
$$

After straightforward calculations one can verify that

$$
d\left\|\hat{\psi}^{(\epsilon)}(t)\right\|_{L^{2}(\mathbb{T})}^{2}=-\frac{2 \gamma_{1}}{\epsilon}\left[\left(\mathfrak{p}_{0}^{(\epsilon)}(t)\right)^{2}-T\right] d t+\left(\frac{2 \gamma_{1} T}{\epsilon}\right)^{1 / 2} \mathfrak{p}_{0}^{(\epsilon)}(t) d w(t), \quad \mathbb{P}_{\epsilon} \text {-a.s. }
$$

Here $\mathbb{P}_{\epsilon}:=\mathbb{P} \otimes \mu_{\epsilon}$. By $\mathbb{E}_{\epsilon}$ we denote the expectation with respect to $\mathbb{P}_{\epsilon}$. From assumptions (2.12) and (2.18) we obtain.

Proposition 2.1 Under the kinetic scaling we have

$$
\mathcal{E}_{*}(t):=\sup _{\epsilon \in(0,1]} \frac{\epsilon}{2} \mathbb{E}_{\epsilon}\left\|\hat{\psi}^{(\epsilon)}(t)\right\|_{L^{2}(\mathbb{T})}^{2} \leq \mathcal{E}_{*}+\gamma_{1} T t, \quad t \geq 0
$$

We can introduce the (averaged) Wigner distribution $W_{\epsilon}(t) \in \mathcal{A}^{\prime}$, corresponding to $\psi^{(\epsilon)}(t)$, by the formula

$$
\left\langle W_{\epsilon}(t), G\right\rangle:=\frac{\epsilon}{2} \sum_{x, x^{\prime} \in \mathbb{Z}} \mathbb{E}_{\epsilon}\left[\left(\psi_{x^{\prime}}^{(\epsilon)}(t)\right)^{\star} \psi_{x}^{(\epsilon)}(t)\right] e_{x^{\prime}-x}(k) G\left(\epsilon \frac{x+x^{\prime}}{2}, k\right), \quad G \in \mathcal{A}
$$


Thanks to (2.19) it is well defined for any $t \geq 0$ and $\epsilon \in(0,1]$. Using the Fourier transform in the first variable we can rewrite the Wigner distribution as

$$
\left\langle W_{\epsilon}(t), G\right\rangle=\int_{\mathbb{T}_{\epsilon} \times \mathbb{T}} \widehat{W}_{\epsilon}^{\star}(t, \eta, k) \widehat{G}(\eta, k) d \eta d k, \quad G \in \mathcal{A},
$$

where

$$
\widehat{W}_{\epsilon}(t, \eta, k):=\frac{\epsilon}{2} \mathbb{E}_{\epsilon}\left[\left(\hat{\psi}^{(\epsilon)}\right)^{\star}\left(t, k-\frac{\epsilon \eta}{2}\right) \hat{\psi}^{(\epsilon)}\left(t, k+\frac{\epsilon \eta}{2}\right)\right] .
$$

We shall refer to $\widehat{W}_{\epsilon}(t)$ as the Fourier-Wigner function corresponding to the given wave function. For the sake of future reference define also $Y_{\epsilon}(t)$, by its Fourier transform

$$
\widehat{Y}_{\epsilon}(t, \eta, k):=\frac{\epsilon}{2} \mathbb{E}_{\epsilon}\left[\hat{\psi}^{(\epsilon)}\left(t,-k+\frac{\epsilon \eta}{2}\right) \hat{\psi}^{(\epsilon)}\left(t, k+\frac{\epsilon \eta}{2}\right)\right] .
$$

\subsection{Kinetic equation}

An important role in our analysis will be played by the function, see Section 2 of [13],

$$
J(t)=\int_{\mathbb{T}} \cos (\omega(k) t) d k
$$

Its Laplace transform

$$
\tilde{J}(\lambda):=\int_{0}^{\infty} e^{-\lambda t} J(t) d t=\int_{\mathbb{T}} \frac{\lambda}{\lambda^{2}+\omega^{2}(k)} d k, \quad \lambda \in \mathbb{C}_{+}:=[z: \operatorname{Re} z>0] .
$$

One can easily see that $\operatorname{Re} \tilde{J}(\lambda)>0$ for $\lambda \in \mathbb{C}_{+}$, therefore we can define the function

$$
\tilde{g}(\lambda):=\left(1+\gamma_{1} \tilde{J}(\lambda)\right)^{-1}, \quad \lambda \in \mathbb{C}_{+} .
$$

We have

$$
|\tilde{g}(\lambda)| \leq 1, \quad \lambda \in \mathbb{C}_{+}
$$

The function $\tilde{g}(\cdot)$ is analytic on $\mathbb{C}_{+}$so, by the Fatou theorem, see e.g. p. 107 of [16], we know that

$$
\nu(k):=\lim _{\epsilon \rightarrow 0+} \tilde{g}(\epsilon-i \omega(k))
$$

exists a.e. in $\mathbb{T}$ and in any $L^{p}(\mathbb{T})$ for $p \in[1, \infty)$.

Let us introduce

$$
\wp(k):=\frac{\gamma_{1} \nu(k)}{2\left|\bar{\omega}^{\prime}(k)\right|}, \quad g(k):=\frac{\gamma_{1}|\nu(k)|^{2}}{\left|\bar{\omega}^{\prime}(k)\right|}, \quad p_{+}(k):=|1-\wp(k)|^{2}, \quad p_{-}(k):=|\wp(k)|^{2},
$$

where $\bar{\omega}^{\prime}(k):=\omega^{\prime}(k) /(2 \pi)$. We have shown in [13] that

$$
\operatorname{Re} \nu(k)=\left(1+\frac{\gamma_{1}}{2\left|\bar{\omega}^{\prime}(k)\right|}\right)|\nu(k)|^{2} .
$$

The functions $p_{ \pm}(\cdot)$ and $g(\cdot)$ are even. Thanks to (2.30) we have

$$
p_{+}(k)+p_{-}(k)+\mathrm{g}(k)=1 \text {. }
$$




\subsubsection{Linear kinetic equation with an interface}

Let $L$ be the operator given by

$$
L F(k):=2 \int_{\mathbb{T}} R\left(k, k^{\prime}\right)\left[F\left(k^{\prime}\right)-F(k)\right] d k^{\prime}, \quad k \in \mathbb{T},
$$

for $F \in L^{1}(\mathbb{T})$ and

$$
\begin{aligned}
& R\left(k, k^{\prime}\right):=\frac{1}{2}\left\{r^{2}\left(k, k-k^{\prime}\right)+r^{2}\left(k, k+k^{\prime}\right)\right\} \\
& =8 \mathfrak{s}^{2}(k) \mathfrak{s}^{2}\left(k^{\prime}\right)\left\{\mathfrak{s}^{2}(k) \mathfrak{c}^{2}\left(k^{\prime}\right)+\mathfrak{s}^{2}\left(k^{\prime}\right) \mathfrak{c}^{2}(k)\right\}, \quad k, k^{\prime} \in \mathbb{T} .
\end{aligned}
$$

Note that (cf (2.10) $)$ the total scattering kernel equals

$$
R(k):=\int_{\mathbb{T}} R\left(k, k^{\prime}\right) d k^{\prime}=\frac{\hat{\theta}(k)}{4} .
$$

Definition 2.2 Given $T \in \mathbb{R}$, let $\mathcal{C}_{T}$ be a subclass of $C_{b}\left(\mathbb{R}_{*} \times \mathbb{T}_{*}\right)$ that consists of continuous functions $F$ that can be continuously extended to $\overline{\mathbb{R}}_{ \pm} \times \mathbb{T}_{*}$ and satisfy the interface conditions

$$
F\left(0^{+}, k\right)=p_{-}(k) F\left(0^{+},-k\right)+p_{+}(k) F\left(0^{-}, k\right)+g(k) T, \quad \text { for } 0<k \leq 1 / 2,
$$

and

$$
F\left(0^{-}, k\right)=p_{-}(k) F\left(0^{-},-k\right)+p_{+}(k) F\left(0^{+}, k\right)+g(k) T, \quad \text { for }-1 / 2<k<0 .
$$

Note that $F \in \mathcal{C}_{T}$ if and only if $F-T^{\prime} \in \mathcal{C}_{T-T^{\prime}}$ for any $T, T^{\prime} \in \mathbb{R}$, because of (2.31).

Let us fix $T \geq 0$. We consider the kinetic interface problem given by equation

$$
\begin{aligned}
& \partial_{t} W(t, y, k)+\bar{\omega}^{\prime}(k) \partial_{y} W(t, y, k)=\gamma_{0} L_{k} W(t, y, k), \quad(t, y, k) \in \mathbb{R}_{+} \times \mathbb{R}_{*} \times \mathbb{T}_{*}, \\
& W(0, y, k)=W_{0}(y, k)
\end{aligned}
$$

with the interface condition

$$
W(t) \in \mathcal{C}_{T}, \quad t \geq 0
$$

Here $L_{k}$ denotes the operator $L$ acting on the $k$ variable. We shall omit writing the subscript if there is no danger of confusion. 


\subsubsection{Simplified case. Explicit solution}

We consider first the situation when equation (2.37) is replaced by

$$
\begin{aligned}
& \partial_{t} W^{\mathrm{un}}(t, y, k)+\bar{\omega}^{\prime}(k) \partial_{y} W^{\mathrm{un}}(t, y, k)=-2 \gamma_{0} R(k) W^{\mathrm{un}}(t, y, k), \quad(t, y, k) \in \mathbb{R}_{+} \times \mathbb{R}_{*} \times \mathbb{T}_{*}, \\
& W^{\mathrm{un}}(0, y, k)=W_{0}(y, k),
\end{aligned}
$$

with the interface conditions (2.35) - (2.36), with $T=0$. It can be solved explicitly, using the method of characteristics, and we obtain

$$
\begin{aligned}
& W^{\mathrm{un}}(t, y, k)=e^{-2 \gamma_{0} R(k) t}\left\{W_{0}\left(y-\bar{\omega}^{\prime}(k) t, k\right) 1_{\left[0, \bar{\omega}^{\prime}(k) t\right] c}(y)+p_{+}(k) W_{0}\left(y-\bar{\omega}^{\prime}(k) t, k\right) 1_{\left[0, \bar{\omega}^{\prime}(k) t\right]}(y)\right. \\
& \left.+p_{-}(k) W_{0}\left(-y+\bar{\omega}^{\prime}(k) t,-k\right) 1_{\left[0, \bar{\omega}^{\prime}(k) t\right]}(y)\right\} .
\end{aligned}
$$

Consider a semigroup of bounded operators on $L^{\infty}\left(\mathbb{R} \times \mathbb{T}_{*}\right)$ defined by

$$
\mathfrak{W}_{t}^{\mathrm{un}}\left(W_{0}\right)(y, k):=W^{\mathrm{un}}(t, y, k),
$$

with $W_{0} \in L^{\infty}\left(\mathbb{R} \times \mathbb{T}_{*}\right), t \geq 0$ and $(y, k) \in \mathbb{R}_{*} \times \mathbb{T}_{*}$. From formula (2.40) one can conclude that $\left(\mathfrak{W}_{t}^{\text {un }}\right)_{t \geq 0}$ forms a semigroup of contractions on both $L^{1}(\mathbb{R} \times \mathbb{T})$ and $L^{\infty}(\mathbb{R} \times \mathbb{T})$. Thus, by interpolation, formula (2.40) defines a semigorup of contractions on any $L^{p}(\mathbb{R} \times \mathbb{T}), 1 \leq p \leq+\infty$.

Note that if $W_{0}$ is continuous in $\mathbb{R}_{*} \times \mathbb{T}_{*}$, then $W^{\text {un }}(t, y, k)$ satisfies the interface conditions (2.35) and (2.36), with $T=0$ for all $t>0$. Therefore, $\left(\mathfrak{W}_{t}^{\text {un }}\right)_{t \geq 0}$ is a semigroup on $\mathcal{C}_{0}$ with $W^{\text {un }}(t, y, k)(\mathrm{cf}$ (2.41) ) satisfying the first equation of (2.39), the interface condition (2.35) - (2.36) and the initial condition

$$
\lim _{t \rightarrow 0+} W^{\mathrm{un}}(t, y, k)=W_{0}(y, k), \quad(y, k) \in \mathbb{R}_{*} \times \mathbb{T}_{*} .
$$

\subsubsection{Kinetic equation - classical solution}

Definition 2.3 We say that a function $W: \overline{\mathbb{R}}_{+} \times \mathbb{R} \times \mathbb{T}_{*} \rightarrow \mathbb{R}$ is a classical solution to equation (2.37) with the interface conditions (2.35), (2.36) at $y=0$, if it is bounded and continuous on $\mathbb{R}_{+} \times \mathbb{R}_{*} \times \mathbb{T}_{*}$, and the following conditions hold:

(1) the restrictions of $W$ to $\mathbb{R}_{+} \times \mathbb{R}_{\iota} \times \mathbb{T}_{\iota^{\prime}}, \iota, \iota^{\prime} \in\{-,+\}$, can be extended to bounded and continuous functions on the respective closures $\overline{\mathbb{R}}_{+} \times \overline{\mathbb{R}}_{\iota} \times \overline{\mathbb{T}}_{\iota^{\prime}}$,

(2) for each $(t, y, k) \in \mathbb{R}_{+} \times \mathbb{R}_{*} \times \mathbb{T}_{*}$ fixed, the function $W\left(t+s, y+\bar{\omega}^{\prime}(k) s, k\right)$ is of the $C^{1}$ class in the $s$-variable in a neighborhood of $s=0$, and the directional derivative

$$
D_{t} W(t, y, k)=\left(\partial_{t}+\bar{\omega}^{\prime}(k) \partial_{y}\right) W(t, y, k):=\left.\frac{d}{d s}\right|_{s=0} W\left(t+s, y+\bar{\omega}^{\prime}(k) s, k\right)
$$


is bounded in $\mathbb{R}_{+} \times \mathbb{R}_{*} \times \mathbb{T}_{*}$ and satisfies

$$
D_{t} W(t, y, k)=\gamma_{0} L_{k} W(t, y, k), \quad(t, y, k) \in \mathbb{R}_{+} \times \mathbb{R}_{*} \times \mathbb{T}_{*},
$$

3) $W(t)$ satisfies (2.35), (2.36) and

$$
\lim _{t \rightarrow 0+} W(t, y, k)=W_{0}(y, k), \quad(y, k) \in \mathbb{R}_{*} \times \mathbb{T}_{*} .
$$

The following result has been shown in [14], see Proposition 2.2.

Proposition 2.4 Suppose that $W_{0} \in \mathcal{C}_{T}$. Then, under the above hypotheses on the scattering kernel $R\left(k, k^{\prime}\right)$ and the dispersion relation $\omega(k)$, there exists a unique classical solution to equation (2.37) with the interface conditions (2.35) and (2.36) in the sense of Definition 2.3.

\subsection{4 $L^{2}$ solution}

We assume that $T=0$. Define $\mathfrak{W}_{t}\left(W_{0}\right):=W(t)$. Thanks to Proposition 2.4 the family $\left(\mathfrak{W}_{t}\right)_{t \geq 0}$ forms a semigroup on the linear space $\mathcal{C}_{0}$. Furthermore, we let

$$
\mathcal{R} F(k):=\int_{\mathbb{T}} R\left(k, k^{\prime}\right) F\left(k^{\prime}\right) d k^{\prime}, \quad k \in \mathbb{T}, \quad F \in L^{1}(\mathbb{T}) .
$$

Let $\mathcal{C}_{0}^{\prime}:=\mathcal{C}_{0} \cap L^{2}(\mathbb{R} \times \mathbb{T})$. The following result holds.

Proposition 2.5 We have $\mathfrak{W}_{t}\left(\mathcal{C}_{0}^{\prime}\right) \subset \mathcal{C}_{0}^{\prime}$ for all $t \geq 0$. The semigroup $\left(\mathfrak{W}_{t}\right)_{t \geq 0}$ extends by the $L^{2}$ closure from $\mathcal{C}_{0}^{\prime}$ to a $C_{0}$-continuous semigroup of contractions on $L^{2}(\mathbb{R} \times \mathbb{T})$. Moreover, it is the unique solution in $L^{2}(\mathbb{R} \times \mathbb{T})$ of the integral equation

$$
\mathfrak{W}_{t}=\mathfrak{W}_{t}^{\mathrm{un}}+2 \gamma_{0} \int_{0}^{t} \mathfrak{W}_{t-s}^{\mathrm{un}} \mathcal{R} \mathfrak{W}_{s} d s, \quad t \geq 0 .
$$

The proof of this result is contained in Appendix $\mathrm{A}$. We shall refer to the semigroup solution described in Proposition 2.5 as the $L^{2}$-solution of quation (2.37) with the interface conditions (2.35) and (2.36) for $T=0$. To extend the definition of such a solution to the case of an arbitrary $T \geq 0$ we proceed as follows. Suppose that $W_{0} \in L^{2}(\mathbb{R} \times \mathbb{T})$. Let $\chi \in C_{c}^{\infty}(\mathbb{R})$ be an arbitrary real valued, even function that satisfies

$$
\chi(y)= \begin{cases}1, & \text { for }|y| \leq 1 / 2 \\ 0, & \text { for }|y| \geq 1 \\ \text { belongs to }[0,1], & \text { if otherwise. }\end{cases}
$$


Definition 2.6 We say that $W(t, y, k)$ is the $L^{2}$-solution of quation (2.37) with the interface conditions (2.35) and (2.36) for a given $T \geq 0$ and an initial condition $W_{0} \in L^{2}(\mathbb{R} \times \mathbb{T})$, if it is of the form

$$
W(t, y, k):=\mathfrak{W}_{t}\left(\widetilde{W}_{0}\right)(y, k)+\int_{0}^{t} \mathfrak{W}_{s}(F)(y, k) d s+T \chi(y), \quad(t, y, k) \in \overline{\mathbb{R}}_{+} \times \mathbb{R} \times \mathbb{T} .
$$

Here

$$
F(y, k):=-T \bar{\omega}^{\prime}(k) \chi^{\prime}(y), \quad \widetilde{W}_{0}(y, k):=W_{0}(y, k)-T \chi(y) .
$$

Remark 2.7 Note that the definition of the solution does not depend on the choice of function $\chi$ satisfying (2.47). Indeed, suppose that $W_{j}, j=1,2$ correspond to two choices of $\chi_{j}(y), j=1,2$, with the respective $\widetilde{W}_{0}^{j}, j=1,2$. Let $\delta W:=W_{2}-W_{1}$. Hence, $\delta W(0)=0$ and $\delta \widetilde{W}_{0}:=\widetilde{W}_{0}^{2}-\widetilde{W}_{0}^{1}=$

$T\left(\chi_{1}-\chi_{2}\right)$ belongs to $\mathcal{C}_{0}$. Moreover, $\frac{d}{d t} \mathfrak{W}_{t}\left(\delta \widetilde{W}_{0}\right)=-\mathfrak{W}_{t}(\delta F)$, where $\delta F:=T\left(\chi_{1}^{\prime}-\chi_{2}^{\prime}\right) \bar{\omega}^{\prime}$. Therefore, using (2.48), we conclude that $\frac{d}{d t} \delta W(t) \equiv 0$, which in turn implies that $W_{j}, j=1,2$ coincide.

Remark 2.8 Suppose that $W_{0} \in \mathcal{C}_{T}$. Then, $W(t, y, k)$ given by(2.48) is the classical solution of (2.37) with the interface conditions (2.35) and (2.36), in the sense of Definition 2.3 ,

\subsection{Asymptotics of the Wigner functions - the statement of the main result}

Thanks to (2.19) we conclude that

$$
\sup _{\epsilon \in(0,1]}\left\|W_{\epsilon}\right\|_{L^{\infty}\left([0, \tau] ; \mathcal{A}^{\prime}\right)}<+\infty, \quad \text { for any } \tau>0
$$

Therefore $\left(W_{\epsilon}(\cdot)\right)$ is sequentially $\star$-weakly compact in $L_{\mathrm{loc}}^{\infty}\left([0,+\infty), \mathcal{A}^{\prime}\right)$, i.e. from any sequence $\epsilon_{n} \rightarrow 0$ we can choose a subsequence, that we still denote by the same symbol, for which $\left(W_{\epsilon_{n}}(\cdot)\right)$ $\star$-weakly converges in $\left(L^{1}([0, t], \mathcal{A})\right)^{\prime}$ for any $t>0$. In our main result we identify the limit as the $L^{2}$ solution of the kinetic equation (2.37) with the interface conditions (2.35) and (2.36), in the sense of Definition 2.6.

Theorem 2.9 Suppose that there exist $C, \kappa>0$ such that

$$
\left|\widehat{W}_{\epsilon}(0, \eta, k)\right|+\left|\widehat{Y}_{\epsilon}(0, \eta, k)\right| \leq C \varphi(\eta), \quad(\eta, k) \in \mathbb{T}_{\epsilon} \times \mathbb{T}, \epsilon \in(0,1]
$$

where

$$
\varphi(\eta):=\frac{1}{\left(1+\eta^{2}\right)^{3 / 2+\kappa}}
$$


and

$$
W_{\epsilon}(0) \underset{\epsilon \rightarrow 0+}{\stackrel{\mathrm{w}^{\star}}{\longrightarrow}} W_{0} \quad \text { in } \mathcal{A}^{\prime} .
$$

Then, $W_{0} \in L^{2}(\mathbb{R} \times \mathbb{T})$ and for any $G \in L_{\text {loc }}^{1}([0,+\infty) ; \mathcal{A})$ we have

$$
\lim _{\epsilon \rightarrow 0+} \int_{0}^{\tau}\left\langle W_{\epsilon}(t), G(t)\right\rangle d t=\int_{0}^{\tau}\langle W(t), G(t)\rangle d t, \quad \tau>0 .
$$

Here $W(t)$ is the $L^{2}$ solution of the kinetic equation (2.37) with the interface conditions (2.35) and (2.36) satisfying $W(0)=W_{0}$.

In the case $T=0$ the theorem is a direct consequence of Theorem 5.5 proved below. The more general case $T \geq 0$ is treated in Section 6 .

\section{Deterministic wave equation corresponding to (2.15)}

Here we consider a deterministic part of the dynamics described in (2.15). Its corresponding energy density function will converge to the solution of (2.39). In microscopic time the evolution of the wave function is given by

$$
\begin{aligned}
& \frac{d}{d t} \hat{\phi}(t, k)=-i \omega(k) \hat{\phi}(t, k)-2 i \epsilon \gamma_{0} R(k) \hat{p}(t, k)-i \gamma_{1} \int_{\mathbb{T}} \hat{p}\left(t, k^{\prime}\right) d k^{\prime}, \\
& \hat{\phi}(0, k)=\hat{\psi}(k),
\end{aligned}
$$

where

$$
\hat{p}(t, k):=\frac{1}{2 i}\left[\hat{\phi}(t, k)-(\hat{\phi}(t,-k))^{\star}\right] .
$$

In fact it is convenient to deal with the vector formulation of the equation for

$$
\hat{\Phi}(t, k)=\left[\begin{array}{c}
\hat{\phi}_{+}(t, k) \\
\hat{\phi}_{-}(t, k),
\end{array}\right], \quad \hat{\Psi}(k)=\left[\begin{array}{c}
\hat{\psi}_{+}(k) \\
\\
\hat{\psi}_{-}(k)
\end{array}\right] .
$$

Here, we use the convention $\hat{\phi}_{+}(t, k)=\hat{\phi}(t, k)$ and $\hat{\phi}_{-}(t, k):=(\hat{\phi}(t,-k))^{\star}$ and similarly for $\hat{\psi}_{ \pm}(k)$. The equation then takes the form

$$
\begin{aligned}
& \frac{d}{d t} \hat{\Phi}(t, k)=\Omega_{\epsilon}(k) \hat{\Phi}(t, k)-i \gamma_{1} \mathfrak{f} p_{0}(t), \\
& \hat{\Phi}(0, k)=\hat{\Psi}(k) .
\end{aligned}
$$

Here

$$
\Omega_{\epsilon}(k):=\left[\begin{array}{cc}
-\gamma_{0} \epsilon R(k)-i \omega(k) & \gamma_{0} \epsilon R(k) \\
\gamma_{0} \epsilon R(k) & -\gamma_{0} \epsilon R(k)+i \omega(k)
\end{array}\right]=\Omega_{0}(k)-\gamma_{0} \epsilon R(k) \mathbf{D}
$$


and

$$
\mathfrak{f}:=\left[\begin{array}{r}
1 \\
-1
\end{array}\right], \quad \mathbf{D}:=\mathfrak{f}^{T} \otimes \mathfrak{f}=\left[\begin{array}{rr}
1 & -1 \\
-1 & 1
\end{array}\right] .
$$

The momentum at $x=0$ equals

$$
p_{0}(t):=\frac{1}{2 i} \int_{\mathbb{T}} \hat{\Phi}(t, k) \cdot \mathfrak{f} d k=\frac{1}{2 i} \int_{\mathbb{T}}\left[\hat{\phi}(t, k)-(\hat{\phi}(t,-k))^{\star}\right] d k .
$$

The eigenvalues of the matrix $\Omega_{\epsilon}(k)$ equal $\lambda_{ \pm}(k)=-\gamma_{0} \epsilon R(k) \pm i \omega_{\epsilon}(k)$, where

$$
\beta(k)=\frac{\gamma_{0} R(k)}{\omega(k)}, \quad \omega_{\epsilon}(k):=\omega(k) \sqrt{1-(\epsilon \beta(k))^{2}} .
$$

Note that $\lambda_{+}^{\star}=\lambda_{-}$.

\section{Solution of $(3.3)$}

By the Duhamel formula, from (3.3) we get

$$
\hat{\Phi}(t, k)=e_{\Omega_{\epsilon}}(k, t) \hat{\Psi}(k)-i \gamma_{1} \int_{0}^{t} e_{\Omega_{\epsilon}}(k, t-s) \mathfrak{f} p_{0}(s) d s .
$$

Here

$$
e_{\Omega_{\epsilon}}(k, t):=\exp \left\{\Omega_{\epsilon}(k) t\right\}=\left[\begin{array}{cc}
e_{\Omega_{\epsilon}}^{1,1}(k, t) & e_{\Omega_{\epsilon}}^{1,2}(k, t) \\
e_{\Omega_{\epsilon}}^{1,2}(k, t) & {\left[e_{\Omega_{\epsilon}}^{1,1}\right]^{\star}(k, t)}
\end{array}\right]
$$

and

$$
\begin{aligned}
& e_{\Omega_{\epsilon}}^{1,1}(k, t):=\frac{1}{4}\left\{\left(1+\sqrt{1-(\epsilon \beta(k))^{2}}\right)^{2} e_{-}(k, t)-(\epsilon \beta(k))^{2} e_{+}(k, t)\right\} \\
& e_{\Omega_{\epsilon}}^{1,2}(k, t):=\frac{i \epsilon \beta(k)}{4}\left(1+\sqrt{1-(\epsilon \beta(k))^{2}}\right)\left(e_{-}(k, t)-e_{+}(k, t)\right), \\
& e_{ \pm}(k, t):=e^{\lambda_{ \pm}(k) t} .
\end{aligned}
$$

Note that $e_{ \pm}^{\star}(k, t)=e_{\mp}(k, t)$.

Multiplying scalarly both sides of (3.7) by $\mathfrak{f}$ and integrating over $k$ we conclude that

$$
p_{0}(t)+\gamma_{1} J_{\epsilon} \star p_{0}(t)=p_{0}^{0}(t)
$$

Here

$$
p_{0}^{0}(t):=\frac{1}{2 i} \int_{\mathbb{T}} e_{\Omega_{\epsilon}}(k, t) \hat{\Psi}(k) \cdot \mathfrak{f} d k
$$

and

$$
J_{\epsilon}(t):=\frac{1}{2} \int_{\mathbb{T}} \exp \left\{\Omega_{\epsilon}(k) t\right\} \mathfrak{f} \cdot \mathfrak{f} d k=\int_{\mathbb{T}} j_{\epsilon}(t, k) d k
$$


where

$$
j_{\epsilon}(t, k):=\frac{1}{2} \exp \left\{\Omega_{\epsilon}(k) t\right\} \mathfrak{f} \cdot \mathfrak{f}=e^{-\epsilon \gamma_{0} R(k) t}\left\{\sqrt{1-(\epsilon \beta(k))^{2}} \cos \left(\omega_{\epsilon}(k) t\right)-\epsilon \beta(k) \sin \left(\omega_{\epsilon}(k) t\right)\right\} .
$$

Taking the Laplace transforms of the both sides of (3.10) we obtain

$$
\tilde{p}_{0}(\lambda)\left(1+\gamma_{1} \tilde{J}_{\epsilon}(\lambda)\right)=\tilde{p}_{0}^{0}(\lambda)
$$

By a direct calculation one concludes that

$$
\operatorname{Re} \tilde{J}_{\epsilon}(\lambda)>0, \quad \text { for any } \lambda \in \mathbb{C}_{+} \text {. }
$$

Since $J_{\epsilon}(\cdot)$ is real valued, we have

$$
\tilde{J}_{\epsilon}^{\star}(\lambda)=\tilde{J}_{\epsilon}\left(\lambda^{\star}\right) \text { for any } \lambda \in \mathbb{C}_{+} .
$$

From (3.14) we get

$$
\tilde{p}_{0}(\lambda)=\tilde{g}_{\epsilon}(\lambda) \tilde{p}_{0}^{0}(\lambda)
$$

with $\tilde{g}_{\epsilon}(\lambda)$ defined by

$$
\tilde{g}_{\epsilon}(\lambda):=\left(1+\gamma_{1} \tilde{J}_{\epsilon}(\lambda)\right)^{-1}, \quad \operatorname{Re} \lambda>0
$$

Thanks to (3.15) we obtain

$$
\left|\tilde{g}_{\epsilon}(\lambda)\right| \leq 1, \quad \operatorname{Re} \lambda>0
$$

and, as a result,

$$
\gamma_{1}\left|\tilde{J}_{\epsilon}(\lambda) \tilde{g}_{\epsilon}(\lambda)\right| \leq 2, \quad \operatorname{Re} \lambda>0
$$

The following result shows in particular that $\tilde{g}_{\epsilon}(\epsilon-i \omega(k))$ approximates in some sense $\nu(k)$, as $\epsilon \rightarrow 0+(\operatorname{see}(\underline{2.28}))$.

Proposition 3.1 Suppose that $K: \mathbb{T} \rightarrow \mathbb{R}_{+}$is a uniformly continuous and bounded function satisfying

$$
\inf _{k \in \mathbb{T}} K(k)>0 .
$$

Then,

$$
\tilde{g}_{\epsilon}(\lambda)=\tilde{g}(\lambda)+\epsilon \tilde{r}_{\epsilon}(\lambda), \quad \lambda \in \mathbb{C}_{+},
$$

where

$$
\lim _{\epsilon \rightarrow 0+} \epsilon^{p} \int_{\mathbb{T}}\left|\tilde{r}_{\epsilon}(\epsilon K(k)-i \omega(k))\right|^{p} d k=0,
$$

for any $p \in[1,+\infty)$. 
The proof of Proposition 3.1 is shown in Appendix C.

Define by $g_{\epsilon}(d s)$ the distribution such that

$$
\tilde{g}_{\epsilon}(\lambda)=\int_{0}^{+\infty} e^{-\lambda t} g_{\epsilon}(d s)
$$

From (3.17) it satisfies

$$
g_{\epsilon}(d s)=\delta(d s)-\gamma_{1} J_{\epsilon} \star g_{\epsilon}(s) d s .
$$

The Volterra equation (3.23) has a unique real-valued solution and $\gamma_{1} J_{\epsilon} \star g_{\epsilon}(s)$ is a $C^{\infty}$ smooth function, see e.g. the argument made in Section 3 of [13]. The solution of (3.7) can be then written as follows

$$
\begin{aligned}
& \hat{\Phi}(t, k)=U(t) \hat{\Psi}(k):=e_{\Omega_{\epsilon}}(k, t) \hat{\Psi}(k)-i \gamma_{1} \int_{0}^{t} e_{\Omega_{\epsilon}}(k, t-s) \mathfrak{f} p_{0}^{0} \star g_{\epsilon}(s) d s \\
& =e_{\Omega_{\epsilon}}(k, t) \hat{\Psi}(k)-\frac{\gamma_{1}}{2} \int_{0}^{t} d s \int_{0}^{s} g_{\epsilon}\left(d s_{1}\right) \int_{\mathbb{T}} e_{\Omega_{\epsilon}}(k, t-s) \mathbf{D} e_{\Omega_{\epsilon}}\left(k, s-s_{1}\right) \hat{\Psi}(\ell) d \ell .
\end{aligned}
$$

\section{Dynamics of the energy density when $T=0$}

Starting with the present section untill Section 6 we shall assume that the thermostat temperature $T=0$, see (2.15). We maintain this assumption untill Section 6 , Let

$$
\hat{\Psi}^{(\epsilon)}(t, k)=\left[\begin{array}{c}
\hat{\psi}_{+}^{(\epsilon)}(t, k) \\
\hat{\psi}_{-}^{(\epsilon)}(t, k)
\end{array}\right]
$$

where $\hat{\psi}_{+}^{(\epsilon)}(t, k):=\hat{\psi}^{(\epsilon)}(t, k)$ and $\hat{\psi}_{-}^{(\epsilon)}(t, k):=\left(\hat{\psi}^{(\epsilon)}\right)^{\star}(t,-k)(\operatorname{cf}(2.14))$. From (2.15) we get

$$
\begin{aligned}
& d \hat{\Psi}^{(\epsilon)}(t, k)=\frac{1}{\epsilon} \Omega_{\epsilon}(k) \hat{\Psi}^{(\epsilon)}(t, k) d t+i \sqrt{\gamma_{0}} \int_{\mathbb{T}} r\left(k, k^{\prime}\right) \mathbf{D} \hat{\Psi}^{(\epsilon)}\left(t, k-k^{\prime}\right) B\left(d t, d k^{\prime}\right)-\frac{i \gamma_{1}}{\epsilon} \mathfrak{p}_{0}(t) \mathfrak{g} d t, \\
& \Psi^{(\epsilon)}(0, k)=\hat{\Psi}(k) .
\end{aligned}
$$

With some abuse of notation we denote by $\mathcal{A}$ the Banach space of all matrix valued functions obtained by the completion of functions of the form

$$
\mathbf{F}(y, k)=\left[\begin{array}{ll}
G(y, k) & H(y, k) \\
H^{\star}(y, k) & G(y,-k)
\end{array}\right], \quad(y, k) \in \mathbb{R} \times \mathbb{T},
$$

with $C^{\infty}$ smooth entries satisfying $G$ is real valued and $H$ is even in $k$. The completion is taken in the norm given by the maximum of the $\mathcal{A}$ norms of the entries, see (2.5). 
The Wigner distribution, corresponding to the wave function $\psi^{(\epsilon)}(t)$, is a $2 \times 2$-matrix tensor $\mathbf{W}_{\epsilon}(t)$, whose entries are distributions, given by their respective Fourier transforms

$$
\begin{aligned}
& \widehat{\mathbf{W}}_{\epsilon}(t, \eta, k):=\frac{\epsilon}{2} \mathbb{E}\left[\hat{\Psi}^{(\epsilon)}\left(t, k+\frac{\epsilon \eta}{2}\right) \otimes\left(\hat{\Psi}^{(\epsilon)}\right)^{\star}\left(t, k-\frac{\epsilon \eta}{2}\right)\right] \\
& =\left[\begin{array}{ll}
\widehat{W}_{\epsilon,+}(t, \eta, k) & \widehat{Y}_{\epsilon,+}(t, \eta, k) \\
\widehat{Y}_{\epsilon,-}(t, \eta, k) & \widehat{W}_{\epsilon,-}(t, \eta, k)
\end{array}\right], \quad(\eta, k) \in \mathbb{T}_{\epsilon} \times \mathbb{T},
\end{aligned}
$$

with

$$
\begin{aligned}
& \widehat{W}_{\epsilon,+}(t, \eta, k):=\widehat{W}_{\epsilon}(t, \eta, k)=\frac{\epsilon}{2} \mathbb{E}_{\epsilon}\left[\hat{\psi}^{(\epsilon)}\left(t, k+\frac{\epsilon \eta}{2}\right)\left(\hat{\psi}^{(\epsilon)}\right)^{\star}\left(t, k-\frac{\epsilon \eta}{2}\right)\right], \\
& \widehat{Y}_{\epsilon,+}(t, \eta, k):=\frac{\epsilon}{2} \mathbb{E}_{\epsilon}\left[\hat{\psi}^{(\epsilon)}\left(t, k+\frac{\epsilon \eta}{2}\right) \hat{\psi}^{(\epsilon)}\left(t,-k+\frac{\epsilon \eta}{2}\right)\right], \\
& \widehat{Y}_{\epsilon,-}(t, \eta, k):=\widehat{Y}_{\epsilon,+}^{\star}(t,-\eta, k), \quad \widehat{W}_{\epsilon,-}(t, \eta, k):=\widehat{W}_{\epsilon,+}(t, \eta,-k) .
\end{aligned}
$$

Then $\mathbf{W}_{\epsilon}(t)$ belongs to $\mathcal{A}^{\prime}$ - the dual to $\mathcal{A}$ that is made of all distributions $\mathbf{W}$, whose Fourier transform in the first variable equals

$$
\widehat{\mathbf{W}}(\eta, k)=\left[\begin{array}{cc}
\widehat{W}_{+}(\eta, k) & \widehat{Y}_{+}(\eta, k) \\
\widehat{Y}_{-}(\eta, k) & \widehat{W}_{-}(\eta, k)
\end{array}\right], \quad(\eta, k) \in \mathbb{T}_{\epsilon} \times \mathbb{T},
$$

whose entries belong to $\mathcal{A}^{\prime}$ and satisfy

$$
\begin{array}{ll}
\widehat{W}_{+}^{\star}(\eta, k)=\widehat{W}_{+}(-\eta, k), & \widehat{Y}_{+}(\eta, k)=\widehat{Y}_{+}(\eta,-k), \\
\widehat{W}_{-}(\eta, k)=\widehat{W}_{+}(\eta,-k), & \widehat{Y}_{-}(\eta, k)=\widehat{Y}_{+}^{\star}(-\eta, k) .
\end{array}
$$

The duality pairing between $\mathcal{A}^{\prime}$ and $\mathcal{A}$ is determined by the relation

$$
\begin{aligned}
& \langle\mathbf{F}, \mathbf{W}\rangle:=\int_{\mathbb{T}_{\epsilon} \times \mathbb{T}} \widehat{\mathbf{F}}(\eta, k) \cdot \mathbf{W}(\eta, k) d \eta d k \\
& =2 \int_{\mathbb{T}_{\epsilon} \times \mathbb{T}}\left\{\widehat{F}_{+}(\eta, k) \widehat{W}_{+}^{\star}(\eta, k)+\operatorname{Re}\left(\widehat{H}_{+}(\eta, k) \widehat{Y}_{+}^{\star}(\eta, k)\right)\right\},
\end{aligned}
$$

wher the scalar product of two matrices is given by

$$
\widehat{\mathbf{F}} \cdot \widehat{\mathbf{W}}=\sum_{\iota= \pm}\left(\widehat{F}_{\iota} \widehat{W}_{\iota}^{\star}+\widehat{H}_{\iota} \widehat{Y}_{\iota}^{\star}\right)
$$

The norm $\|\mathbf{W}\|_{\mathcal{A}^{\prime}}$ is therefore the sum of the norm of its entries.

Thanks to (2.12) and (2.19) we conclude that

$$
\sup _{\epsilon \in(0,1]} \sup _{t \geq 0}\left\|\mathbf{W}_{\epsilon}(t)\right\|_{\mathcal{A}^{\prime}}=: A_{*}^{\prime}<+\infty \text {. }
$$


Therefore $\left(\mathbf{W}_{\epsilon}(\cdot)\right)$ is bounded in $L^{\infty}\left([0,+\infty), \mathcal{A}^{\prime}\right)$. In consequence, from any sequence $\epsilon_{n} \rightarrow 0$ we can choose a subsequence, that we still denote by the same symbol, such that $\left(\mathbf{W}_{\epsilon_{n}}(\cdot)\right)$ is *-weakly convergent in $\left(L^{1}([0,+\infty), \mathcal{A})\right)^{\prime}$.

In what follows we shall also consider the Hilbert spaces $\mathcal{L}_{2, \epsilon}$ with the scalar product $\langle\cdot, \cdot\rangle_{\mathcal{L}_{2, \epsilon}}$ given by the formula (4.7). The respective Hilbert space norms are

$$
\|\mathbf{W}\|_{\mathcal{L}_{2, \epsilon}}:=\left\{2\left(\left\|W_{+}\right\|_{\mathcal{L}_{2, \epsilon}}^{2}+\left\|Y_{+}\right\|_{\mathcal{L}_{2, \epsilon}}^{2}\right)\right\}^{1 / 2}
$$

We introduce the following notation, given a function $f: \mathbb{T} \rightarrow \mathbb{C}$, we let

$$
\bar{f}(k, \eta):=\frac{1}{2}\left[f\left(k+\frac{\eta}{2}\right)+f\left(k-\frac{\eta}{2}\right)\right]
$$

and the difference quotient for the dispersion relation

$$
\delta_{\epsilon} \omega(k, \eta):=\frac{1}{\epsilon}\left[\omega\left(k+\frac{\epsilon \eta}{2}\right)-\omega\left(k-\frac{\epsilon \eta}{2}\right)\right] .
$$

Equipped with this notation we introduce

$$
\widehat{\mathbf{H}}_{\epsilon}(\eta, k)=\left[\begin{array}{cc}
-i \delta_{\epsilon} \omega(k ; \eta) & -\frac{2 i}{\epsilon} \bar{\omega}(k, \epsilon \eta) \\
\frac{2 i}{\epsilon} \bar{\omega}(k, \epsilon \eta) & i \delta_{\epsilon} \omega(k ; \eta)
\end{array}\right], \quad(\eta, k) \in \mathbb{T}_{\epsilon} \times \mathbb{T}
$$

and

$$
\begin{aligned}
& L_{\eta} f(k):=2 \mathcal{R}_{\eta} f(k)-2 \bar{R}(k, \eta) f(k), \quad L_{\eta}^{ \pm} f(k):=2 \mathcal{R}_{\eta} f(k)-2 R\left(k \pm \frac{\eta}{2}\right) f(k), \\
& \mathcal{R}_{\eta} f(k):=\int_{\mathbb{T}} R\left(k, k^{\prime}, \eta\right) f\left(k^{\prime}\right) d k^{\prime}, \\
& R\left(k, k^{\prime}, \ell\right):=\frac{1}{2} \sum_{\iota= \pm 1} r\left(k-\frac{\ell}{2}, k-\iota k^{\prime}\right) r\left(k+\frac{\ell}{2}, k-\iota k^{\prime}\right), \quad k, k^{\prime} \in \mathbb{T}, \ell \in 2 \mathbb{T} .
\end{aligned}
$$

We denote by $\mathfrak{L}_{\epsilon \eta}, \mathfrak{H}_{\epsilon}, \mathfrak{T}_{\epsilon}$ the operators, acting on $\mathcal{L}_{2, \epsilon}$, defined by

$$
\widehat{\mathfrak{L}_{\epsilon \eta} \mathbf{W}}=\widehat{\mathfrak{L}}_{\epsilon \eta} \widehat{\mathbf{W}}, \quad \widehat{\mathfrak{H}_{\epsilon} \mathbf{W}}=\widehat{\mathfrak{H}}_{\epsilon} \widehat{\mathbf{W}}, \quad \widehat{\mathfrak{T}_{\epsilon} \mathbf{W}}=\widehat{\mathfrak{T}}_{\epsilon} \widehat{\mathbf{W}}
$$

Here $\widehat{\mathbf{W}}$ is the Fourier transform of $\mathbf{W} \in \mathcal{L}_{2, \epsilon}$, given by (4.6). Operator $\hat{\mathfrak{H}}_{\epsilon}$, acting on $L^{2}\left(\mathbb{T}_{\epsilon} \times \mathbb{T}\right)$, is given by

$$
\widehat{\mathfrak{H}}_{\epsilon} \widehat{\mathbf{W}}(\eta, k):=\widehat{\mathbf{H}}_{\epsilon}(\eta, k) \circ \widehat{\mathbf{W}}(\eta, k)=\left[\begin{array}{cc}
-i \delta_{\epsilon} \omega(k ; \eta) \widehat{W}_{+}(\eta, k) & -\frac{2 i}{\epsilon} \bar{\omega}(k, \epsilon \eta) \widehat{Y}_{+}(\eta, k) \\
\frac{2 i}{\epsilon} \bar{\omega}(k, \epsilon \eta) \widehat{Y}_{-}(\eta, k) & i \delta_{\epsilon} \omega(k ; \eta) \widehat{W}_{-}(\eta, k)
\end{array}\right],
$$


with $\circ$ denoting the Hadamard's product of $2 \times 2$ matrices. Moreover, $\widehat{\mathfrak{L}}_{\epsilon \eta}$ and $\widehat{\mathfrak{T}}_{\epsilon}$ act on $L^{2}\left(\mathbb{T}_{\epsilon} \times \mathbb{T}\right)$ via the formulas

$$
\widehat{\mathfrak{L}}_{\epsilon \eta} \widehat{\mathbf{W}}(\eta, k):=\left[\begin{array}{cc}
\widehat{W}_{+}^{\prime}(\eta, k) & \widehat{Y}_{+}^{\prime}(\eta, k) \\
\widehat{Y}_{-}^{\prime}(\eta, k) & \widehat{W}_{-}^{\prime}(\eta, k)
\end{array}\right], \quad \widehat{\mathfrak{T}}_{\epsilon} \widehat{\mathbf{W}}(\eta, k):=\left[\begin{array}{cc}
\widehat{W}_{+}^{\prime \prime}(\eta, k) & \widehat{Y}_{+}^{\prime \prime}(\eta, k) \\
\widehat{Y}_{-}^{\prime \prime}(\eta, k) & \widehat{W}_{-}^{\prime \prime}(\eta, k)
\end{array}\right],
$$

with

$$
\begin{aligned}
& \widehat{W}_{ \pm}^{\prime}(\eta, k)=L_{\epsilon \eta} \widehat{W}_{ \pm}(\eta, k)-\frac{1}{2} \sum_{\iota= \pm} L_{\iota \epsilon \eta}^{ \pm} \widehat{Y}_{-\iota}(\eta, k) \\
& \widehat{Y}_{ \pm}^{\prime}(\eta, k)=L_{\epsilon \eta} \widehat{Y}_{ \pm}(\eta, k)+\mathcal{R}_{\epsilon \eta}\left(\widehat{Y}_{\mp}-\widehat{Y}_{ \pm}\right)(\eta, k)-\frac{1}{2} \sum_{\iota= \pm} L_{\iota \epsilon \eta}^{ \pm} \widehat{W}_{-\iota}(\eta, k)
\end{aligned}
$$

and

$$
\begin{aligned}
& \widehat{W}_{ \pm}^{\prime \prime}(\eta, k)=\frac{1}{2 \epsilon} \int_{\mathbb{T}}\left[\widehat{Y}_{ \pm}\left(\eta-\frac{2 k^{\prime}}{\epsilon}, k+k^{\prime}\right)+\widehat{Y}_{\mp}\left(\eta+\frac{2 k^{\prime}}{\epsilon}, k+k^{\prime}\right)\right. \\
& \left.-\widehat{W}_{ \pm}\left(\eta-\frac{2 k^{\prime}}{\epsilon}, k+k^{\prime}\right)-\widehat{W}_{ \pm}\left(\eta+\frac{2 k^{\prime}}{\epsilon}, k+k^{\prime}\right)\right] d k^{\prime} \\
& \widehat{Y}_{ \pm}^{\prime \prime}(\eta, k)=-\frac{1}{2 \epsilon} \int_{\mathbb{T}}\left[\widehat{Y}_{ \pm}\left(\eta+\frac{2 k^{\prime}}{\epsilon}, k+k^{\prime}\right)+\widehat{Y}_{ \pm}\left(\eta-\frac{2 k^{\prime}}{\epsilon}, k+k^{\prime}\right)\right. \\
& \left.-\widehat{W}_{\mp}\left(\eta+\frac{2 k^{\prime}}{\epsilon}, k+k^{\prime}\right)-\widehat{W}_{ \pm}\left(\eta-\frac{2 k^{\prime}}{\epsilon}, k+k^{\prime}\right)\right] d k^{\prime} .
\end{aligned}
$$

Using (4.2) we obtain the following system of equations for the evolution of the tensor $\widehat{\mathbf{W}}_{\epsilon}(t, \eta, k)$ :

$$
\frac{d}{d t} \widehat{\mathbf{W}}_{\epsilon}(t, \eta, k)=\left(\gamma_{0} \widehat{\mathfrak{L}}_{\epsilon \eta}+\widehat{\mathfrak{H}}_{\epsilon}+\gamma_{1} \widehat{\mathfrak{T}}_{\epsilon}\right) \widehat{\mathbf{W}}_{\epsilon}(t, \eta, k)
$$

The respective semigroups on $\mathcal{L}_{2, \epsilon}$ and $L^{2}\left(\mathbb{T}_{\epsilon} \times \mathbb{T}\right)$ shall be denoted by

$$
\mathfrak{W}_{\epsilon}(t):=\exp \left\{\left(\gamma_{0} \mathfrak{L}_{\epsilon \eta}+\mathfrak{H}_{\epsilon}+\gamma_{1} \mathfrak{T}_{\epsilon}\right) t\right\}, \quad \widehat{\mathfrak{W}}_{\epsilon}(t):=\exp \left\{\left(\gamma_{0} \hat{\mathfrak{L}}_{\epsilon \eta}+\hat{\mathfrak{H}}_{\epsilon}+\gamma_{1} \hat{\mathfrak{T}}_{\epsilon}\right) t\right\}, \quad t \geq 0
$$

Let us introduce the Hilbert space norms

$$
\|\mathbf{W}\|_{\mathcal{H}_{0, \epsilon}}:=\left\{\int_{\mathbb{T}_{\epsilon} \times \mathbb{T}} R\left(k-\frac{\epsilon \eta}{2}\right)\left|\widehat{W}_{+}(\eta, k)-\widehat{Y}_{+}(\eta, k)\right|^{2} d \eta d k\right\}^{1 / 2}
$$

and

$$
\|\mathbf{W}\|_{\mathcal{H}_{1, \epsilon}}:=\left\{\int_{\mathbb{T}} d k\left|\int_{\mathbb{T}_{\epsilon}}\left[\widehat{W}_{+}\left(t, \eta, k-\frac{\epsilon \eta}{2}\right)-\widehat{Y}_{+}\left(t, \eta, k-\frac{\epsilon \eta}{2}\right)\right] d \eta\right|^{2}\right\}^{1 / 2} .
$$


By a direct calculation one can verify the following identity

$$
\begin{aligned}
& \left\|\mathbf{W}_{\epsilon}(t)\right\|_{\mathcal{L}_{2, \epsilon}}^{2}+8 \gamma_{0} \int_{0}^{t}\left\|\mathbf{W}_{\epsilon}(s)\right\|_{\mathcal{H}_{0, \epsilon}}^{2} d s+4 \gamma_{1} \int_{0}^{t}\left\|\mathbf{W}_{\epsilon}(s)\right\|_{\mathcal{H}_{1, \epsilon}}^{2} d s=\left\|\mathbf{W}_{\epsilon}(0)\right\|_{\mathcal{L}_{2, \epsilon}}^{2} \\
& +4 \gamma_{0} \int_{0}^{t} d s \int_{\mathbb{T}_{\epsilon} \times \mathbb{T}^{2}} R\left(k, k^{\prime}, \epsilon \eta\right) \operatorname{Re}\left\{2 \widehat{W}_{\epsilon,+}(s, \eta, k)\left(\widehat{W}_{\epsilon,+}\right)^{\star}\left(s, \eta, k^{\prime}\right)\right. \\
& \left.+Y_{\epsilon,+}(s, \eta, k)\left(\widehat{Y}_{\epsilon,+}\right)^{\star}\left(s, \eta, k^{\prime}\right)+Y_{\epsilon,-}(s, \eta, k)\left(\widehat{Y}_{\epsilon,+}\right)^{\star}\left(s, \eta, k^{\prime}\right)\right\} d \eta d k d k^{\prime} \\
& -8 \gamma_{0} \int_{0}^{t} d s \int_{\mathbb{T}_{\epsilon} \times \mathbb{T}^{2}} R\left(k, k^{\prime}, \epsilon \eta\right) \operatorname{Re}\left\{\widehat{W}_{\epsilon,+}(s, \eta, k)\left(\widehat{Y}_{\epsilon,+}\right)^{\star}\left(s, \eta, k^{\prime}\right)\right. \\
& \left.+\widehat{Y}_{\epsilon,+}(s, \eta, k)\left(\widehat{W}_{\epsilon,+}\right)^{\star}\left(s, \eta, k^{\prime}\right)\right\} d \eta d k d k^{\prime}, \quad t \geq 0, \epsilon \in(0,1] .
\end{aligned}
$$

In particular we have

$$
\frac{d}{d t}\left\|\mathbf{W}_{\epsilon}(t)\right\|_{\mathcal{L}_{2, \epsilon}}^{2} \leq 16 R_{*}\left\|\mathbf{W}_{\epsilon}(t)\right\|_{\mathcal{L}_{2, \epsilon}}^{2}
$$

and

$$
2 \gamma_{0}\left\|\mathbf{W}_{\epsilon}(t)\right\|_{\mathcal{H}_{0, \epsilon}}^{2}+\gamma_{1}\left\|\mathbf{W}_{\epsilon}(t)\right\|_{\mathcal{H}_{1, \epsilon}}^{2} \leq 4 R_{*}\left\|\mathbf{W}_{\epsilon}(t)\right\|_{\mathcal{L}_{2, \epsilon}}^{2},
$$

where $R_{*}:=\sup _{k, k^{\prime} \in \mathbb{T}, \ell \in 2 \mathbb{T}}\left|R\left(k, k^{\prime}, \ell\right)\right|$. By the Gronwall inequality we conclude the following.

Proposition 4.1 We have

$$
\left\|\mathbf{W}_{\epsilon}(t)\right\|_{\mathcal{L}_{2, \epsilon}} \leq\left\|\mathbf{W}_{\epsilon}(0)\right\|_{\mathcal{L}_{2, \epsilon}} e^{8 R_{*} t}
$$

and

$$
2 \gamma_{0}\left\|\mathbf{W}_{\epsilon}(t)\right\|_{\mathcal{H}_{0, \epsilon}}^{2}+\gamma_{1}\left\|\mathbf{W}_{\epsilon}(t)\right\|_{\mathcal{H}_{1, \epsilon}}^{2} \leq 4 R_{*}\left\|\mathbf{W}_{\epsilon}(0)\right\|_{\mathcal{L}_{2, \epsilon}}^{2} e^{16 R_{*} t}, \quad t \geq 0
$$

A direct consequence of Proposition 4.1 is the following.

Corollary 4.2 The semigroup $\left(\mathfrak{W}_{\epsilon}(t)\right)_{t \geq 0}$ is uniformly continuous on $\mathcal{L}_{2, \epsilon}$. Moreover, its norm satisfies

$$
\left\|\mathfrak{W}_{\epsilon}(t)\right\|_{\mathcal{L}_{2, \epsilon}} \leq e^{8 R_{*} t}, \quad t \geq 0, \quad \epsilon \in(0,1] .
$$

Corollary 4.3 Suppose that $\mathbf{W}(\cdot)$ is a ${ }^{\star}$-weak limit of $\left(\mathbf{W}_{\epsilon_{n}}(\cdot)\right)$ in $\left(L^{1}([0,+\infty), \mathcal{A})\right)^{\prime}$ and assume that

$$
W_{*}:=\limsup _{\epsilon \rightarrow 0+}\left\|\mathbf{W}_{\epsilon}(0)\right\|_{\mathcal{L}_{2, \epsilon}}<+\infty .
$$

Then, $\mathbf{W}(\cdot) \in L_{\mathrm{loc}}^{\infty}\left([0,+\infty) ; L^{2}(\mathbb{R} \times \mathbb{T})\right)$. In fact we have

$$
\|\mathbf{W}(\cdot)\|_{L^{\infty}\left([0, \tau] ; L^{2}(\mathbb{R} \times \mathbb{T})\right)} \leq e^{8 R_{*} \tau} W_{*}, \quad \tau \geq 0 .
$$


Proof. Fix some $\tau>0$. Suppose that $\mathbf{G} \in \mathcal{A}_{c}$. Suppose that $A \subset[0, \tau]$ is a Borel measurable set. We know that

$$
\lim _{n \rightarrow+\infty} \int_{A} d u \int_{\mathbb{R} \times \mathbb{T}} \widehat{\mathbf{G}}(\eta, k) \cdot \widehat{\mathbf{W}}_{\epsilon_{n}}(u, \eta, k) d \eta d k=\int_{A} d u \int_{\mathbb{R} \times \mathbb{T}} \widehat{\mathbf{G}}(\eta, k) \cdot \widehat{\mathbf{W}}(u, \eta, k) \hat{d} \eta d k .
$$

Suppose that $n_{0}$ is such that $\operatorname{supp} \widehat{\mathbf{G}} \subset\left[-\epsilon_{n}^{-1}, \epsilon_{n}^{-1}\right] \times \mathbb{T}, n \geq n_{0}$. Then for these $n$ we can write

$$
\begin{aligned}
& \left|\int_{A} d u \int_{\mathbb{R} \times \mathbb{T}} \widehat{\mathbf{G}}(\eta, k) \cdot \widehat{\mathbf{W}}_{\epsilon_{n}}(u, \eta, k) d \eta d k\right|=\left|\int_{A} d u \int_{\mathbb{T}_{\epsilon_{n}} \times \mathbb{T}} \widehat{\mathbf{G}}(\eta, k) \cdot \widehat{\mathbf{W}}_{\epsilon_{n}}(u, \eta, k) d \eta d k\right| \\
& \leq\|\mathbf{G}\|_{L^{2}(\mathbb{R} \times \mathbb{T})} \int_{A}\left\|\mathbf{W}_{\epsilon_{n}}(u)\right\|_{\mathcal{L}_{2, \epsilon_{n}}} d u .
\end{aligned}
$$

Therefore, by (4.26),

$$
\left|\int_{A} d u \int_{\mathbb{R} \times \mathbb{T}} \widehat{\mathbf{G}}(\eta, k) \cdot \widehat{\mathbf{W}}(u, \eta, k) d \eta d k\right| \leq e^{8 R_{*} \tau} m_{1}(A)\|\mathbf{G}\|_{L^{2}(\mathbb{R} \times \mathbb{T})} \limsup _{\epsilon \rightarrow 0+}\left\|\mathbf{W}_{\epsilon}(0)\right\|_{\mathcal{L}_{2, \epsilon}} .
$$

By the density argument, the above inequality holds for all $\mathbf{G} \in L^{2}(\mathbb{R} \times \mathbb{T})$. We can further extend the above estimate by taking a simple function of the form $\mathbf{F}(t):=\sum_{i=1}^{n} \mathbf{G}_{i} 1_{A_{i}}(t)$, where $\mathbf{G}_{i} \in \mathcal{A}_{2}$ and $A_{1}, \ldots, A_{n}$ are disjoint, Borel measurable subsets of $[0, \tau]$. The above argument generalizes easily and we conclude that

$$
\left|\int_{0}^{\tau} \int_{\mathbb{R} \times \mathbb{T}} \widehat{\mathbf{F}}(u, \eta, k) \cdot \widehat{\mathbf{W}}(u, \eta, k) d \eta d k\right| \leq e^{8 R_{*} \tau}\|\mathbf{F}\|_{L^{1}\left([0, \tau]: L^{2}(\mathbb{R} \times \mathbb{T})\right)} \limsup _{\epsilon \rightarrow 0+}\left\|\mathbf{W}_{\epsilon}(0)\right\|_{\mathcal{L}_{2, \epsilon}} .
$$

Since the functions $\mathbf{F}(\cdot)$ are dense in $L^{1}\left([0, \tau] ; L^{2}(\mathbb{R} \times \mathbb{T})\right)$ we conclude the proof of the corollary. Estimate (4.30) is a consequence of the results of Section IV.1 of [10].

\section{Fourier-Wigner functions for the deterministic dynamics}

Throughout the present section we shall assume that $T=0$.

\subsection{Dynamics of the Wigner distributions for the solution of (3.1)}

We consider the Wigner tensor $\mathbf{W}_{\epsilon}^{\text {un }}(t)$, corresponding to the wave function $\hat{\Phi}^{(\epsilon)}(t, k)=\hat{\Phi}(t / \epsilon, k)$, where $\hat{\Phi}(t, k)$ is given by (3.2). Its Fourier transform (in the first variable) is given by (see (3.24)

$$
\widehat{\mathbf{W}}_{\epsilon}^{\mathrm{un}}(t, \eta, k):=\frac{\epsilon}{2} \mathbb{E}\left[\hat{\Phi}^{(\epsilon)}\left(t, k+\frac{\epsilon \eta}{2}\right) \otimes\left(\hat{\Phi}^{(\epsilon)}\right)^{\star}\left(t, k-\frac{\epsilon \eta}{2}\right)\right]=\left[\begin{array}{cc}
\widehat{W}_{\epsilon,+}^{\mathrm{un}}(t, \eta, k) & \widehat{Y}_{\epsilon,+}^{\mathrm{un}}(t, \eta, k) \\
\widehat{Y}_{\epsilon,-}^{\mathrm{un}}(t, \eta, k) & \widehat{W}_{\epsilon,-}^{\mathrm{un}}(t, \eta, k)
\end{array}\right],
$$


with

$$
\begin{aligned}
& \widehat{W}_{\epsilon,+}^{\text {un }}(t, \eta, k):=\frac{\epsilon}{2} \mathbb{E}\left[\hat{\phi}^{(\epsilon)}\left(t, k+\frac{\epsilon \eta}{2}\right)\left(\hat{\phi}^{(\epsilon)}\right)^{\star}\left(t, k-\frac{\epsilon \eta}{2}\right)\right], \\
& \widehat{Y}_{\epsilon,+}^{\text {un }}(t, \eta, k):=\frac{\epsilon}{2} \mathbb{E}\left[\hat{\phi}^{(\epsilon)}\left(t, k+\frac{\epsilon \eta}{2}\right) \hat{\phi}^{(\epsilon)}\left(t,-k+\frac{\epsilon \eta}{2}\right)\right], \\
& \widehat{Y}_{\epsilon,-}^{\text {un }}(t, \eta, k):=\left(\widehat{Y}_{\epsilon,+}^{\text {un }}(t,-\eta, k)\right)^{\star}, \quad \widehat{W}_{\epsilon,-}^{\text {un }}(t, \eta, k):=\widehat{W}_{\epsilon,+}^{\text {un }}(t, \eta,-k) .
\end{aligned}
$$

Using (3.3) we conclude that the dynamics of the tensor $\mathbf{W}_{\epsilon}^{\mathrm{un}}(t)$ is described by the $\mathcal{L}_{2, \epsilon}$ strongly continuous semigroup

$$
\mathfrak{W}_{\epsilon}^{\mathrm{un}}(t):=\exp \left\{\left(\gamma_{0} \mathfrak{L}_{\epsilon \eta}^{\prime}+\mathfrak{H}_{\epsilon}+\gamma_{1} \mathfrak{T}_{\epsilon}\right) t\right\}, \quad t \geq 0
$$

where $\mathfrak{H}_{\epsilon}, \mathfrak{T}_{\epsilon}$ are defined in (4.15), (4.16) and (4.18). On the other hand $\mathfrak{L}_{\epsilon \eta}^{\prime}$ is given by the respective Fourier transform

$$
\widehat{\mathfrak{L}}_{\epsilon \eta}^{\prime} \widehat{\mathbf{W}}(\eta, k):=\left[\begin{array}{cc}
\widehat{W}_{+}^{\prime}(\eta, k) & \widehat{Y}_{+}^{\prime}(\eta, k) \\
\widehat{Y}_{-}^{\prime}(\eta, k) & \widehat{W}_{-}^{\prime}(\eta, k)
\end{array}\right],
$$

with

$$
\begin{aligned}
& \widehat{W}_{ \pm}^{\prime}(\eta, k):=-2 \bar{R}(k, \epsilon \eta) \widehat{W}_{ \pm}^{\mathrm{un}}(\eta, k)+\left\{R\left(k \mp \frac{\epsilon \eta}{2}\right) \widehat{Y}_{+}^{\mathrm{un}}(\eta, k)+R\left(k \pm \frac{\epsilon \eta}{2}\right) \widehat{Y}_{-}^{\mathrm{un}}(\eta, k)\right\} \\
& \widehat{Y}_{ \pm}^{\prime}(\eta, k)=-2 \bar{R}(k, \epsilon \eta) \widehat{Y}_{ \pm}(\eta, k)+R\left(k \mp \frac{\epsilon \eta}{2}\right) \widehat{W}_{+}(\eta, k)+R\left(k \pm \frac{\epsilon \eta}{2}\right) \widehat{W}_{-}(\eta, k) .
\end{aligned}
$$

By a direct calculation we can verify the following.

Proposition 5.1 The following identity holds

$$
\left\|\mathbf{W}_{\epsilon}^{\mathrm{un}}(t)\right\|_{\mathcal{L}_{2, \epsilon}}^{2}+8 \gamma_{0} \int_{0}^{t}\left\|\mathbf{W}_{\epsilon}^{\mathrm{un}}(s)\right\|_{\mathcal{H}_{0, \epsilon}}^{2} d s+4 \gamma_{1} \int_{0}^{t}\left\|\mathbf{W}_{\epsilon}^{\mathrm{un}}(s)\right\|_{\mathcal{H}_{1, \epsilon}}^{2} d s=\left\|\mathbf{W}_{\epsilon}^{\mathrm{un}}(0)\right\|_{\mathcal{L}_{2, \epsilon}}^{2}, \quad t \geq 0, \epsilon \in(0,1] .
$$

A direct consequence of the proposition is the following.

Corollary $5.2\left(\mathfrak{W}_{\epsilon}^{\mathrm{un}}(t)\right)_{t \geq 0}$ forms a uniformly continuous semigroup of contractions on $\mathcal{L}_{2, \epsilon}$ for any $\epsilon \in(0,1]$.

Let $p_{0}^{(\epsilon)}(t):=p_{0}(t / \epsilon)($ see $(3.5))$ and

$$
d_{\epsilon}(t, k):=i \mathbb{E}_{\epsilon}\left[\left(\hat{\phi}^{(\epsilon)}\right)^{\star}(t, k) p_{0}^{(\epsilon)}(t)\right] .
$$

By a direct calculation we obtain

$$
\left\|\mathbf{W}_{\epsilon}^{\mathrm{un}}(t)\right\|_{\mathcal{H}_{1, \epsilon}}=2\left\|d_{\epsilon}(t)\right\|_{L^{2}(\mathbb{T})}, \quad t \geq 0, \epsilon \in(0,1] .
$$

From (5.4) it follows directly. 
Corollary 5.3 We have

$$
\int_{0}^{+\infty}\left\|d_{\epsilon}(t)\right\|_{L^{2}(\mathbb{T})}^{2} d t \leq \frac{\left\|\mathbf{W}_{\epsilon}(0)\right\|_{\mathfrak{Q}_{2, \epsilon}}^{2}}{16 \gamma_{1}}, \quad \epsilon \in(0,1] .
$$

\subsection{Duhamel representation of the energy density dynamics}

Using the Duhamel formula we can reformulate (4.2) as follows

$$
\hat{\Psi}^{(\epsilon)}(t, k)=U\left(\frac{t}{\epsilon}\right) \hat{\Psi}(k)+\sqrt{\gamma_{0}} \int_{0}^{t} U\left(\frac{t-s}{\epsilon}\right)\left(i \int_{\mathbb{T}} r\left(\cdot, k^{\prime}\right) \mathbf{D} \hat{\Psi}^{(\epsilon)}\left(s, \cdot-k^{\prime}\right) B\left(d s, d k^{\prime}\right)\right) .
$$

Here $U(t)$ is given by (3.2) and (3.3). Using (5.8) to express the Fourier-Wigner tensor $\mathbf{W}_{\epsilon}(t)$ we obtain the following equality

$$
\mathbf{W}_{\epsilon}(t)=\mathbf{W}_{\epsilon}^{\mathrm{un}}(t)+\mathbf{W}_{\epsilon}^{\prime}(t)
$$

where

$$
\mathbf{W}_{\epsilon}^{\mathrm{un}}(t)=\mathfrak{W}_{\epsilon}^{\mathrm{un}}(t)\left(\mathbf{W}_{\epsilon}(0)\right)
$$

and

$$
\widehat{\mathbf{W}}_{\epsilon}^{\prime}(t, \eta, k)=\frac{\epsilon \gamma_{0}}{2} \sum_{n} \int_{0}^{t} \mathbb{E}\left\{\left(U\left(\frac{t-s}{\epsilon}\right)\left(\hat{\Psi}_{n}^{\epsilon}(s)\right)\right)^{\star}\left(k-\frac{\epsilon \eta}{2}\right) \otimes U\left(\frac{t-s}{\epsilon}\right)\left(\hat{\Psi}_{n}^{\epsilon}(s)\right)\left(k+\frac{\epsilon \eta}{2}\right)\right\} d s .
$$

Here

$$
\hat{\Psi}_{n}^{(\epsilon)}(s, k)=i \int_{\mathbb{T}} r\left(k, k^{\prime}\right) \mathbf{D} \hat{\Psi}^{(\epsilon)}\left(s, k-k^{\prime}\right) e_{n}\left(k^{\prime}\right) d k^{\prime},
$$

where $\left(e_{n}\right)$ is an orthonormal base in $L^{2}(\mathbb{T})$. Using (3.24) we conclude that

$$
\widehat{\mathbf{W}}_{\epsilon}^{\prime}(t, \eta, k)=\gamma_{0} \int_{0}^{t} \widehat{\mathfrak{W}}_{\epsilon}^{\mathrm{un}}(t-s)\left(\mathbf{V}_{\epsilon}(s)\right)(\eta, k) d s .
$$

Here $\widehat{\mathfrak{W}}_{\epsilon}^{\text {un }}(t)$ is the Fourier transform of the semigroup (5.1) and $\mathbf{V}_{\epsilon}(t)$ is given by its Fourier transform $\widehat{\mathbf{V}}_{\epsilon}(t, \eta, k):=\widehat{\mathfrak{R}}_{\epsilon} \widehat{\mathbf{W}}_{\epsilon}(t, \eta, k)$, where $\widehat{\Re}_{\epsilon}: \hat{\mathcal{L}}_{2, \epsilon} \rightarrow \hat{\mathcal{L}}_{2, \epsilon}$ is defined by

$\widehat{\Re}_{\epsilon} \widehat{\mathbf{W}}:=\widehat{V}_{\epsilon}(\eta, k) \mathbf{D}$,

$\widehat{V}_{\epsilon}(\eta, k)=\int_{\mathbb{T}} r\left(k-\frac{\epsilon \eta}{2}, k-k^{\prime}\right) r\left(k+\frac{\epsilon \eta}{2}, k-k^{\prime}\right)\left[\widehat{W}_{+}\left(\eta, k^{\prime}\right)+\widehat{W}_{-}\left(\eta, k^{\prime}\right)-\widehat{Y}_{+}\left(\eta, k^{\prime}\right)-\widehat{Y}_{-}\left(\eta, k^{\prime}\right)\right] d k^{\prime}$.

Summarizing, we have shown that

$$
\mathbf{W}_{\epsilon}(t)=\mathfrak{W}_{\epsilon}^{\mathrm{un}}(t)\left(\mathbf{W}_{\epsilon}(0)\right)+\gamma_{0} \int_{0}^{t} \mathfrak{W}_{\epsilon}^{\mathrm{un}}(t-s)\left(\mathfrak{R}_{\epsilon} \mathbf{W}_{\epsilon}(s)\right)(\eta, k) d s, \quad t \geq 0, \epsilon \in(0,1] .
$$

Operator $\mathfrak{R}_{\epsilon}$ is given by its Fourier transform, see (5.11). A direct calculation yields the following. 
Proposition 5.4 Operators $\mathfrak{R}_{\epsilon}$ can be defined by (5.11) as bounded operators both on $\mathcal{L}_{2, \epsilon}$ and $L^{2}(\mathbb{R} \times \mathbb{T})$. They are uniformly bounded in $\epsilon \in(0,1]$. More precisely, there exists $\mathfrak{r}_{*}>0$ such that

$$
\left\|\mathfrak{R}_{\epsilon}\right\|_{\mathcal{L}_{2, \epsilon}} \leq \mathfrak{r}_{*}, \quad\left\|\mathfrak{R}_{\epsilon}\right\|_{L^{2}(\mathbb{R} \times \mathbb{T})} \leq \mathfrak{r}_{*}, \quad \epsilon \in(0,1]
$$

In addition, we have

$$
\lim _{\epsilon \rightarrow 0+} \mathfrak{R}_{\epsilon} \mathbf{W}=\mathfrak{R} \mathbf{W}, \quad \text { in } L^{2}(\mathbb{R} \times \mathbb{T})
$$

for any $\mathbf{W} \in L^{2}(\mathbb{R} \times \mathbb{T})$. Here $($ cf (2.45) $)$

$$
\mathfrak{R} \mathbf{W}(y, k)=\mathbf{D} \mathcal{R}\left(W_{+}(y, \cdot)+W_{-}(y, \cdot)-Y_{+}(y, \cdot)-Y_{-}(y, \cdot)\right)(k), \quad(y, k) \in \mathbb{R} \times \mathbb{T}
$$

Theorem 2.9 is a direct corollary from the following.

Theorem 5.5 Under the assumptions of Theorem 2.9 for any $G \in L^{1}([0,+\infty) ; \mathcal{A})$ we have

$$
\lim _{\epsilon \rightarrow 0+} \int_{0}^{+\infty} d t \int_{\mathbb{R} \times \mathbb{T}} \widehat{Y}_{\epsilon, \pm}^{\star}(t, \eta, k) \widehat{G}(t, \eta, k) d \eta d k=0
$$

and

$$
\lim _{\epsilon \rightarrow 0+} \int_{0}^{+\infty} d t \int_{\mathbb{R} \times \mathbb{T}} \widehat{W}_{\epsilon, \pm}^{\star}(t, \eta, k) \widehat{G}(t, \eta, k) d \eta d k=\int_{0}^{+\infty} d t \int_{\mathbb{R} \times \mathbb{T}} \widehat{W}^{\star}(t, \eta, \pm k) \widehat{G}(t, \eta, k) d \eta d k
$$

where $W(t, y, k)$ is the unique solution of the equation (2.46) with the initial condition $W_{0}$.

The proof is presented in Section 5.4.

\subsection{Laplace transform}

As we have already mentioned, see (4.9),$\left(\mathbf{W}_{\epsilon}(\cdot)\right)$ is *-weakly sequentially compact in the dual to $L^{1}([0,+\infty), \mathcal{A})$, therefore the proof of Theorem 5.5 comes down to showing uniqueness of limiting points, as $\epsilon \rightarrow 0+$. For that purpose it is convenient to work with the Laplace transform

$$
\mathbf{w}_{\epsilon}(\lambda)=\left[\begin{array}{cc}
w_{\epsilon,+}(\lambda) & y_{\epsilon,+}(\lambda) \\
y_{\epsilon,-}(\lambda) & w_{\epsilon,-}(\lambda)
\end{array}\right]:=\int_{0}^{+\infty} e^{-\lambda t} \mathbf{W}_{\epsilon}(t) d t .
$$

Sometimes, when we wish to highlight the dependence on the initial data, we shall also write

$$
\mathbf{W}_{\epsilon}\left(\lambda ; \mathbf{W}_{\epsilon}(0)\right)=: \widetilde{\mathfrak{W}}_{\epsilon}(\lambda)\left(\mathbf{W}_{\epsilon}(0)\right)
$$

Thanks to (2.19) the Laplace transform is well defined as an element in $\mathcal{A}^{\prime}$ for any $\operatorname{Re} \lambda>0$. From Proposition 4.1 we conclude the following estimate

$$
\left\|\widetilde{\mathfrak{W}}_{\epsilon}(\lambda)\right\|_{\mathcal{L}_{2, \epsilon}} \leq\left[\left(\operatorname{Re} \lambda-8 R_{*}\right) \operatorname{Re} \lambda\right]^{-1 / 2}, \quad \operatorname{Re} \lambda>8 R_{*} .
$$


The argument used in the proof of Corollary 4.3 shows that

$$
\mathbf{w}(\lambda)=\left[\begin{array}{ll}
w_{+}(\lambda) & y_{+}(\lambda) \\
y_{-}(\lambda) & w_{-}(\lambda)
\end{array}\right]
$$

- any ${ }^{\star}$-weak limiting point in $\mathcal{A}^{\prime}$ of $\mathbf{w}_{\epsilon}(\lambda)$, as $\epsilon \rightarrow 0+$ - belongs to $L^{2}(\mathbb{R} \times \mathbb{T})$ and satisfies the estimate

$$
\|\mathbf{w}(\lambda)\|_{L^{2}(\mathbb{R} \times \mathbb{T})} \leq\left[\left(\operatorname{Re} \lambda-8 R_{*}\right) \operatorname{Re} \lambda\right]^{-1 / 2} \limsup _{\epsilon \rightarrow 0+}\left\|\mathbf{W}_{\epsilon}(0)\right\|_{L^{2}(\mathbb{R} \times \mathbb{T})}, \quad \operatorname{Re} \lambda>8 R_{*} .
$$

Equation (5.12) leads to the following equation for the Laplace transform

$$
\mathbf{w}_{\epsilon}\left(\lambda ; \mathbf{W}_{\epsilon}(0)\right)=\mathbf{w}_{\epsilon}^{\mathrm{un}}\left(\lambda ; \mathbf{W}_{\epsilon}(0)\right)+\gamma_{0} \mathbf{w}_{\epsilon}^{\mathrm{un}}\left(\lambda ; \mathbf{v}_{\epsilon}(\lambda)\right), \quad \operatorname{Re} \lambda>0 .
$$

Here,

$$
\mathbf{w}_{\epsilon}^{\mathrm{un}}(\lambda ; \mathbf{W}):=\widetilde{\mathfrak{W}}_{\epsilon}^{\mathrm{un}}(\lambda) \mathbf{W}:=\int_{0}^{+\infty} e^{-\lambda t} \mathfrak{W}_{\epsilon}^{\mathrm{un}}(t) \mathbf{W} d t, \quad \mathbf{W} \in \mathcal{L}_{2, \epsilon}
$$

and

$$
\mathbf{v}_{\epsilon}(\lambda):=\Re_{\epsilon} \mathbf{W}_{\epsilon}\left(\lambda ; \mathbf{W}_{\epsilon}(0)\right)
$$

Let $W_{0} \in L^{2}(\mathbb{R} \times \mathbb{T})$. We define

$$
w_{+}^{\mathrm{un}}\left(\lambda, W_{0}\right)=\widetilde{\mathfrak{W}}^{\mathrm{un}}(\lambda) W_{0}:=\int_{0}^{+\infty} e^{-\lambda t} \mathfrak{W}_{t}^{\mathrm{un}} W_{0}(\eta, k) d t
$$

It follows directly from (2.40) that its Fourier transform satisfies the equality

$$
\begin{aligned}
& \left(\lambda+2 \gamma_{0} R(k)+i \omega^{\prime}(k) \eta\right) \widehat{w}_{+}^{\text {un }}\left(\lambda, \eta, k ; W_{0}\right)=\widehat{W}_{0}(\eta, k) \\
& -\gamma_{1}\left\{\left(1-p_{+}(k)\right) \int_{\mathbb{R}} \frac{\widehat{W}_{0}\left(\eta^{\prime}, k\right) d \eta^{\prime} d k}{\lambda+2 \gamma_{0} R(k)+i \omega^{\prime}(k) \eta^{\prime}}-p_{-}(k) \int_{\mathbb{R}} \frac{\widehat{W}_{0}\left(\eta^{\prime},-k\right) d \eta^{\prime}}{\lambda+2 \gamma_{0} R(k)-i \omega^{\prime}(k) \eta^{\prime}}\right\} .
\end{aligned}
$$

It is clear from the above formula that $w_{+}^{\text {un }}\left(\lambda, W_{0}\right) \in \mathcal{A}^{\prime}$, provided that $W_{0} \in \mathcal{A}^{\prime}$. Thanks to formulas (2.29) and (2.30) it can also be seen that $w_{+}^{\text {un }}\left(\lambda, W_{0}\right) \in L^{2}(\mathbb{R} \times \mathbb{T})$, if $W_{0} \in L^{2}(\mathbb{R} \times \mathbb{T})$.

The first step towards the limit identification of $\left(\mathbf{W}_{\epsilon}(\cdot)\right)$ consists in showing the following result.

Theorem 5.6 Under the assumptions on the initial data made in Theorem [2.9, the family $\left(\mathbf{w}_{\epsilon}^{\text {un }}\left(\lambda ; \mathbf{W}_{\epsilon}(0)\right)\right)$ converges ${ }^{\star}$-weakly to $\mathbf{w}^{\mathrm{un}}\left(\lambda ; W_{0}\right)$ of the form

$$
\mathbf{w}^{\mathrm{un}}\left(\lambda, y, k ; W_{0}\right)=\left[\begin{array}{cc}
w_{+}^{\mathrm{un}}\left(\lambda, y, k ; W_{0}\right) & 0 \\
0 & w_{+}^{\mathrm{un}}\left(\lambda, y,-k ; W_{0}\right)
\end{array}\right], \quad(y, k) \in \mathbb{R} \times \mathbb{T},
$$

where the Fourier transform of $w_{+}^{\mathrm{un}}\left(\lambda, y, k ; W_{0}\right)$ is given by (5.24). 
The proof of this result follows closely the argument contained in [13]. We present its outline in Appendix B.

The identification of the limit of $\left(\mathbf{W}_{\epsilon}(t)\right)$ is possible thanks to the following result.

Theorem 5.7 Assume the hypotheses of Theorem 2.9. Furthermore, suppose that $\mathbf{w}(\lambda)$ is the *weak limit of $\left(\mathbf{w}_{\epsilon_{n}}(\lambda)\right)$ in $\mathcal{A}^{\prime}$ for some sequence $\epsilon_{n} \rightarrow 0+$. Then, there exists $\lambda_{0}>0$ such that $\mathbf{w}(\lambda) \in L^{2}(\mathbb{R} \times \mathbb{T})$ for $\operatorname{Re} \lambda>\lambda_{0}$ and

$$
\mathbf{w}(\lambda, y, k)=\left[\begin{array}{cc}
w_{+}(\lambda, y, k) & 0 \\
0 & w_{+}(\lambda, y,-k)
\end{array}\right], \quad(y, k) \in L^{2}(\mathbb{R} \times \mathbb{T}),
$$

where $w_{+}(\lambda, y, k)$ satisfies the equation

$$
w_{+}(\lambda)=w_{+}^{\mathrm{un}}\left(\lambda ; W_{0}\right)+2 \gamma_{0} \widetilde{\mathfrak{W}}^{\mathrm{un}}(\lambda) \mathcal{R} w_{+}(\lambda),
$$

and $\mathcal{R}: L^{2}(\mathbb{R} \times \mathbb{T}) \rightarrow L^{2}(\mathbb{R} \times \mathbb{T})(c f(2.45))$ is given by

$$
\mathcal{R} F(y, k):=\int_{\mathbb{T}} R\left(k, k^{\prime}\right) F\left(y, k^{\prime}\right) d k^{\prime}, \quad(y, k) \in \mathbb{R} \times \mathbb{T}, \quad F \in L^{2}(\mathbb{R} \times \mathbb{T}) .
$$

We present the proof of Theorem [5.7 in Section 5.5 below.

\subsection{Proof of Theorem 5.5}

Since $\left(\mathfrak{W}_{t}^{\text {un }}\right)_{t \geq 0}$ is a semigroup of contractions, see Proposition 2.5, we have

$$
\left\|\widetilde{\mathfrak{W}}^{\mathrm{un}}(\lambda)\right\|_{L^{2}(\mathbb{R} \times \mathbb{T})} \leq \frac{1}{\operatorname{Re} \lambda}, \quad \operatorname{Re} \lambda>0
$$

Thanks to the fact that $\mathcal{R}$ is bounded, it is straightforward to see that equation (5.26) has a unique $L^{2}(\mathbb{R} \times \mathbb{T})$ solution for $\lambda$ with a sufficiently large real part. Therefore, the Laplace transform $w_{+}(\lambda)$ of any limiting point of $\left(W_{\epsilon}(t)\right)$ is uniquely determined by (5.26). This in turn implies the conclusion of the theorem.

\subsection{Proof of Theorem 5.7}

To avoid using double subscript notation we assume that $\mathbf{w}(\lambda)=\lim _{\epsilon \rightarrow 0+} \mathbf{w}_{\epsilon}(\lambda)$. We wish to show that the limit is of the form (5.25) with $w_{+}(\lambda)$ satisfying (5.26). 


\subsubsection{Proof of (5.25)}

We prove that

$$
\lim _{\epsilon \rightarrow 0+} \int_{\mathbb{R} \times \mathbb{T}} \widehat{y}_{\epsilon, \iota}^{\star}(\lambda, \eta, k) \widehat{G}(\eta, k) d \eta d k=0, \quad \iota= \pm
$$

for any $G \in \mathcal{A}_{c}$ (see (2.4) $)$ and $\operatorname{Re} \lambda>0$. Consider only the case of $\iota=+$, the other one is analogous. Assume that $K>0$ is fixed and $\epsilon$ is so small that

$$
\operatorname{supp} \hat{G} \subset[-K, K] \times \mathbb{T} \subset\left[\epsilon^{-1}, \epsilon^{-1}\right] \times \mathbb{T} \text {. }
$$

Let

$$
\chi_{K}(\eta, k):=1_{[-K, K]}(\eta) 1_{\mathbb{T}}(k) .
$$

By virtue of estimate (5.19) we conclude that $\left(\widehat{y}_{\epsilon,+}(\lambda) \chi_{K}\right)$ is bounded, thus weakly compact in $L^{2}(\mathbb{R} \times \mathbb{T})$ for a fixed $K>0$. It converges weakly in $L^{2}(\mathbb{R} \times \mathbb{T})$, due to its *- weak convergence in $\mathcal{A}^{\prime}$. Denote the limit, belonging to $L^{2}(\mathbb{R} \times \mathbb{T})$, by $\widehat{y}_{+}(\lambda) \chi_{K}$.

Let

$$
\begin{aligned}
& \mathfrak{d}_{\epsilon}(\lambda, k):=i \int_{0}^{+\infty} e^{-\lambda t} \mathbb{E}_{\epsilon}\left[\left(\hat{\psi}^{(\epsilon)}\right)^{\star}(t, k) \mathfrak{p}_{0}^{(\epsilon)}(t)\right] d t \\
& =\frac{1}{2} \int_{\mathbb{T}_{\epsilon}}\left[\widehat{y}_{\epsilon,+}\left(\lambda, \eta, k-\frac{\epsilon \eta}{2}\right)-\widehat{w}_{\epsilon,+}\left(\lambda, \eta, k-\frac{\epsilon \eta}{2}\right)\right] d \eta .
\end{aligned}
$$

Taking the Laplace transforms of both sides of (4.19) and multiplying by $\chi_{K}$ we obtain in particular the equation

$$
\begin{aligned}
& -\epsilon \gamma_{0} R\left(k-\frac{\epsilon \eta}{2}\right) \widehat{w}_{\epsilon,+}(\lambda) \chi_{K}+\left(\epsilon \lambda+2 \gamma_{0} \epsilon \bar{R}(k, \epsilon \eta)+2 i \bar{\omega}(k, \epsilon \eta)\right) \widehat{y}_{\epsilon,+}(\lambda) \chi_{K}-\gamma_{0} \epsilon R\left(k+\frac{\epsilon \eta}{2}\right) \widehat{w}_{\epsilon,-}(\lambda) \chi_{K} \\
& =\epsilon \widehat{Y}_{\epsilon,+}(\eta, k) \chi_{K}+\frac{\epsilon \gamma_{1}}{2}\left\{\mathfrak{d}_{\epsilon}^{\star}\left(\lambda,-k+\frac{\epsilon \eta}{2}\right)+\mathfrak{d}_{\epsilon}^{\star}\left(\lambda, k+\frac{\epsilon \eta}{2}\right)\right\} \chi_{K} \\
& +\gamma_{0} \mathcal{R}_{\epsilon \eta}\left\{\widehat{y}_{\epsilon,+}(\lambda)+\widehat{y}_{\epsilon,-}(\lambda)-\widehat{w}_{\epsilon,+}(\lambda)-\widehat{w}_{\epsilon,-}(\lambda)\right\} \chi_{K} .
\end{aligned}
$$

Thanks to estimate (4.27), see also (4.22), we conclude that

$$
\left\|\mathfrak{d}_{\epsilon}(\lambda)\right\|_{L^{2}(\mathbb{T})} \leq\left(\frac{R_{*}}{\gamma_{1} \operatorname{Re} \lambda\left(\operatorname{Re} \lambda-8 R_{*}\right)}\right)^{1 / 2}\left\|\mathbf{W}_{\epsilon}(0)\right\|_{\mathcal{L}_{2, \epsilon}}, \quad \epsilon \in(0,1], \operatorname{Re} \lambda>8 R_{*} .
$$

From the strong convergence in $L^{2}(\mathbb{R} \times \mathbb{T})$ of the coefficients of (5.33) and estimate (5.34) we conclude that $2 i \omega(k) \widehat{y}_{+}(\lambda) \chi_{K}=0$ for any $\lambda$ such that $\operatorname{Re} \lambda>8 R_{*}$. This in turn implies that $\widehat{y}_{+}(\lambda)=0$ for such $\lambda$-s, which by analytic continuation, implies (5.29). 


\subsubsection{Proof of $(\underline{5.26})$}

To avoid writing double subscript we maintain the convention to denote a subsequence of $\left(\mathbf{w}_{\epsilon}(\lambda)\right)$ by the same symbol as the entire sequence. From the already proved part of the theorem we know that the limiting element is of the form (5.25). We let the test matrix valued function from $\mathcal{A}_{c}$ be of the form

$$
\mathbf{G}(y, k):=\left[\begin{array}{cc}
G(y, k) & 0 \\
0 & G(y,-k)
\end{array}\right], \quad(y, k) \in \mathbb{R} \times \mathbb{T},
$$

with $G$ satisfying (5.30). Using the inclusion $[-K, K] \subset \mathbb{T}_{\epsilon}$ we can treat $\mathbf{G}$ as an element of $\mathcal{L}_{2, \epsilon}$. Applying both sides of (5.21) to this test matrix we obtain the following equality

$$
\left\langle\mathbf{w}_{\epsilon}(\lambda), \mathbf{G}\right\rangle_{\mathcal{L}_{2, \epsilon}}=I_{\epsilon}+I I_{\epsilon}
$$

where

$$
I_{\epsilon}:=\left\langle\mathbf{w}_{\epsilon}^{\mathrm{un}}(\lambda), \mathbf{G}\right\rangle_{\mathcal{L}_{2, \epsilon}}, \quad I I_{\epsilon}:=\gamma_{0}\left\langle\mathbf{w}_{\epsilon}(\lambda), \mathfrak{R}_{\epsilon}^{\star}\left(\widetilde{\mathfrak{W}}_{\epsilon}^{\mathrm{un}}(\lambda)\right)^{\star} \mathbf{G}\right\rangle_{\mathcal{L}_{2, \epsilon}} .
$$

Here $\mathfrak{R}_{\epsilon}^{\star}$ and $\left(\widetilde{\mathfrak{W}}_{\epsilon}^{\text {un }}(\lambda)\right)^{\star}$ are the adjoints of the respective operators (see (15.11) and (5.22) $)$, in $\mathcal{L}_{2, \epsilon}$. Invoking Theorem 5.6 we conclude that

$$
\lim _{\epsilon \rightarrow 0+} I_{\epsilon}=2 \int_{\mathbb{R} \times \mathbb{T}}\left(w_{+}^{\mathrm{un}}\left(\lambda, y, k ; W_{0}\right)\right)^{\star} G(y, k) d y d k, \quad \operatorname{Re} \lambda>0 .
$$

Concerning the term $I I_{\epsilon}$, we are going to show that there exists $\lambda_{0}>0$ such that

$$
\lim _{\epsilon \rightarrow 0+} I I_{\epsilon}=4 \gamma_{0} \int_{\mathbb{R} \times \mathbb{T}} w_{+}(\lambda, k, y) \mathcal{R} \bar{g}_{+}(\lambda)(y, k) d y d k
$$

for $\operatorname{Re} \lambda>\lambda_{0}$. Here

$$
\bar{g}_{+}(\lambda, y, k):=\left(\widetilde{\mathfrak{W}}^{\mathrm{un}}(\lambda)\right)^{\star}(G)(y, k) .
$$

Denote by

$$
\widehat{\mathrm{g}}_{\epsilon}(\lambda, \eta, k):=\left[\begin{array}{cc}
\widehat{g}_{\epsilon,+}(\lambda, \eta, k) & \widehat{h}_{\epsilon,+}(\lambda, \eta, k) \\
\widehat{h}_{\epsilon,-}(\lambda, \eta, k) & \widehat{g}_{\epsilon,-}(\lambda, \eta, k)
\end{array}\right],
$$

the Fourier transform of the distribution $\mathbf{g}_{\epsilon}(\lambda)=\left(\widetilde{\mathfrak{W}}_{\epsilon}^{\mathrm{un}}(\lambda)\right)^{\star} \mathbf{G}$ and let

$$
\widehat{\mathbf{f}}_{\epsilon}(\lambda, \eta, k):=\widehat{\mathfrak{R}}_{\epsilon}^{\star} \widehat{\mathrm{g}}_{\epsilon}(\lambda)(\eta, k), \quad \epsilon \in(0,1] .
$$

Note that

$$
\begin{aligned}
& \widehat{\mathbf{f}}_{\epsilon}(\lambda, \eta, k)=\widehat{f}_{\epsilon}(\lambda, \eta, k) \mathbf{D}, \text { with } \\
& \widehat{f}_{\epsilon}(\lambda, \eta, k):=\int_{\mathbb{T}} r\left(k^{\prime}-\frac{\epsilon \eta}{2}, k^{\prime}-k\right) r\left(k^{\prime}+\frac{\epsilon \eta}{2}, k^{\prime}-k\right) \\
& \times\left[g_{\epsilon,+}\left(\lambda, \eta, k^{\prime}\right)+g_{\epsilon,-}\left(\lambda, \eta, k^{\prime}\right)-h_{\epsilon,+}\left(\lambda, \eta, k^{\prime}\right)-h_{\epsilon,-}\left(\lambda, \eta, k^{\prime}\right)\right] d k^{\prime} .
\end{aligned}
$$


The argument used in the proof of Theorem [5.6 (it suffices only to replace the terms containing the dispersion relation by their conjugates) shows that in fact

$$
\lim _{\epsilon \rightarrow 0+}\left\langle\mathbf{g}_{\epsilon}(\lambda), \mathbf{F}\right\rangle=\langle\overline{\mathbf{g}}(\lambda), \mathbf{F}\rangle
$$

for any $\mathbf{F} \in \mathcal{A}_{c}$ and $\operatorname{Re} \lambda>0$. Here

$$
\overline{\mathbf{g}}(\lambda, y, k):=\left[\begin{array}{cc}
\bar{g}_{+}(\lambda, y, k) & 0 \\
0 & \bar{g}_{+}(\lambda, y,-k)
\end{array}\right]
$$

Suppose now that $\varphi$ is an arbitrary, bounded and compactly supported measurable function. Then, using (5.40), we conclude that

$$
\lim _{\epsilon \rightarrow 0+} \varphi(\eta) \widehat{\mathbf{f}}_{\epsilon}(\lambda, \eta, k)=2 \varphi(\eta) \mathbf{D} \mathcal{R} \widehat{\bar{g}}_{+}(\lambda, \eta, k), \quad \text { weakly in } L^{2}(\mathbb{R} \times \mathbb{T}),
$$

where $\widehat{\bar{g}}_{+}(\lambda)$ is the Fourier transform of $\bar{g}_{+}(\lambda)$ in the first variable.

In order to show (5.37), we prove the following two results.

Lemma 5.8 There exists $C>0$ such that

$$
\left\|\nabla \widehat{\mathbf{f}}_{\epsilon}(\lambda)\right\|_{L^{2}\left(\mathbb{T}_{\epsilon} \times \mathbb{T}\right)} \leq \frac{C}{\operatorname{Re} \lambda}, \quad \epsilon \in(0,1], \operatorname{Re} \lambda>0 .
$$

The gradient operator in (5.42) is in the $\eta$ and $k$ variables.

Lemma 5.9 For any $\rho>0$ there exists $M>0$ such that

$$
\limsup _{\epsilon \rightarrow 0+} \int_{\left[(\eta, k) \in \mathbb{T}_{\epsilon} \times \mathbb{T},|\eta|>M\right]}\left|\widehat{\mathbf{w}}_{\epsilon}(\lambda, \eta, k) \cdot \widehat{\mathbf{f}}_{\epsilon}(\lambda, \eta, k)\right| d \eta d k<\rho
$$

Having the above results we can write that $I I_{\epsilon}=I I_{\epsilon, 1}+I I_{\epsilon, 2}$, where

$$
\begin{aligned}
& I I_{\epsilon, 1}:=\int_{\mathbb{T}_{\epsilon} \times \mathbb{T}} \varphi^{2}(\eta) \widehat{\mathbf{w}}_{\epsilon}(\lambda, \eta, k) \cdot \widehat{\mathbf{f}}_{\epsilon}(\lambda, \eta, k) d \eta d k, \\
& I I_{\epsilon, 2}:=\int_{\mathbb{T}_{\epsilon} \times \mathbb{T}}\left[1-\varphi^{2}(\eta)\right] \widehat{\mathbf{w}}_{\epsilon}(\lambda, \eta, k) \cdot \widehat{\mathbf{f}}_{\epsilon}(\lambda, \eta, k) d \eta d k .
\end{aligned}
$$

Here $\varphi: \mathbb{R} \rightarrow[0,1]$ is a $C^{\infty}$ smooth function satisfying $\varphi(\eta) \equiv 1,|\eta| \leq M$ and $\varphi(\eta) \equiv 0,|\eta| \geq 2 M$.

Choose an arbitrary $\rho>0$. Using Lemma 5.9 we conclude that $M$ can be adjusted in such a way that

$$
\limsup _{\epsilon \rightarrow 0+}\left|I I_{\epsilon, 2}\right|<\rho
$$


On the other hand, by virtue of Lemma 5.8 the set $\left(\varphi \widehat{\mathbf{f}}_{\epsilon}(\lambda)\right), \epsilon \in(0,1]$ is compact in $L^{2}(\mathbb{R} \times \mathbb{T})$ in the strong topology. Since it converges also in the weak topology (see (5.41)), it has to also converge strongly in $L^{2}(\mathbb{R} \times \mathbb{T})$. Combining this with the fact that $\left(\varphi \widehat{\mathbf{w}}_{\epsilon}(\lambda)\right)$ is weakly compact in $L^{2}(\mathbb{R} \times \mathbb{T})$ we conclude that

$$
\limsup _{\epsilon \rightarrow 0+} I I_{\epsilon, 1}=4 \int_{\mathbb{R} \times \mathbb{T}} \varphi^{2}(\eta) \widehat{w}_{+}(\lambda, \eta, k) d \eta d k\left\{\int_{\mathbb{T}} R\left(k, k^{\prime}\right) \widehat{\bar{g}}_{+}^{\star}\left(\lambda, \eta, k^{\prime}\right) d k^{\prime}\right\} .
$$

This ends the proof of (5.37). The only items yet to be proven are Lemmas 5.8 and 5.9 ,

\subsubsection{Proof of Lemma 5.8}

Let $\mathbf{G}_{\epsilon}(t):=\left(\mathfrak{W}_{\epsilon}^{\text {un }}\right)^{\star}(t) \mathbf{G}$ and $\widehat{\mathbf{G}}_{\epsilon}(t, \eta, k)$ be its Fourier transform in the first variable. From (5.1) we conclude that it satisfies

$$
\frac{d}{d t} \widehat{\mathbf{G}}_{\epsilon}(t)=\left(\gamma_{0} \widehat{\mathfrak{L}}_{\epsilon \eta}^{\prime}+\widehat{\mathfrak{H}}_{\epsilon}^{\star}+\gamma_{1} \widehat{\mathfrak{T}}_{\epsilon}\right) \widehat{\mathbf{G}}_{\epsilon}(t)
$$

One can formulate the respective the energy balance equation, see (5.4),

$$
\left\|\widehat{\mathbf{G}}_{\epsilon}(t)\right\|_{L^{2}\left(\mathbb{T}_{\epsilon} \times \mathbb{T}\right)}^{2}+8 \gamma_{0} \int_{0}^{t}\left\|\mathbf{G}_{\epsilon}(s)\right\|_{\mathcal{H}_{0, \epsilon}}^{2} d s+4 \gamma_{1} \int_{0}^{t}\left\|\mathbf{G}_{\epsilon}(s)\right\|_{\mathcal{H}_{1, \epsilon}}^{2} d s=\|\widehat{\mathbf{G}}\|_{L^{2}\left(\mathbb{T}_{\epsilon} \times \mathbb{T}\right)}^{2}, \quad t \geq 0
$$

and $\epsilon$ sufficiently small that (5.30) holds. It allows us to obtain estimates

$$
\left\|\widehat{\mathbf{g}}_{\epsilon}(\lambda)\right\|_{L^{2}\left(\mathbb{T}_{\epsilon} \times \mathbb{T}\right)} \leq \frac{1}{\operatorname{Re} \lambda}\|\mathbf{G}\|_{L^{2}(\mathbb{R} \times \mathbb{T})}
$$

for $\operatorname{Re} \lambda>0$ and $\epsilon$ sufficiently small, as above.

Thanks to (5.39) and estimate (5.48) we conclude that

$$
\left\|\partial_{k} \widehat{\mathbf{f}}_{\epsilon}(\lambda)\right\|_{L^{2}\left(\mathbb{T}_{\epsilon} \times \mathbb{T}\right)} \leq \frac{R_{*}^{\prime}\|\mathbf{G}\|_{L^{2}(\mathbb{R} \times \mathbb{T})}}{\operatorname{Re} \lambda}, \quad \epsilon \in(0,1], \operatorname{Re} \lambda>0
$$

with $R_{*}^{\prime}:=\sup _{k, k^{\prime} \in \mathbb{T}, \ell \in 2 \mathbb{T}}\left|\partial_{k^{\prime}} R\left(k, k^{\prime}, \ell\right)\right|(\operatorname{cf}(4.13)$ ).

To estimate the $L^{2}$-norm of $\partial_{\eta} \widehat{\mathbf{f}}_{\epsilon}(\lambda, \eta, k)$ we differentiate in $\eta$ both sides of (5.46) and obtain

$$
\frac{d}{d t} \widehat{\mathbf{G}}_{\epsilon, \eta}^{\prime}(t)=\left(\gamma_{0} \widehat{\mathfrak{L}}_{\epsilon \eta}^{\prime}+\widehat{\mathfrak{H}}_{\epsilon}^{\star}+\gamma_{1} \widehat{\mathfrak{T}}_{\epsilon}\right) \widehat{\mathbf{G}}_{\epsilon, \eta}^{\prime}(t)+\left(\gamma_{0} \partial_{\eta} \widehat{\mathfrak{L}}_{\epsilon \eta}^{\prime}+\partial_{\eta} \widehat{\mathfrak{H}}_{\epsilon}^{\star}\right) \widehat{\mathbf{G}}_{\epsilon}(t)
$$

Both here and below

$$
\widehat{\mathbf{G}}_{\epsilon, \eta}^{\prime}(t):=\partial_{\eta} \widehat{\mathbf{G}}_{\epsilon}(t)=\left[\begin{array}{cc}
\widehat{G}_{\epsilon, \eta,+}(t, \eta, k) & \widehat{H}_{\epsilon, \eta,+}(t, \eta, k) \\
\widehat{H}_{\epsilon, \eta,-}(t, \eta, k) & \widehat{G}_{\epsilon, \eta,-}(t, \eta, k)
\end{array}\right]
$$


and $\widehat{\mathbf{G}}_{\eta}^{\prime}:=\partial_{\eta} \widehat{\mathbf{G}}$. Let $\mathbf{G}_{\epsilon}^{(1)}(t)$ denote the inverse Fourier transform of $\widehat{\mathbf{G}}_{\epsilon, \eta}^{\prime}(t)$. Analogously to (5.4) we conclude the following identity

$$
\begin{aligned}
\left\|\widehat{\mathbf{G}}_{\epsilon, \eta}^{\prime}(t)\right\|_{L^{2}\left(\mathbb{T}_{\epsilon} \times \mathbb{T}\right)}^{2}+8 \gamma_{0} \int_{0}^{t}\left\|\mathbf{G}_{\epsilon}^{(1)}(s)\right\|_{\mathcal{H}_{0, \epsilon}}^{2} d s+4 \gamma_{1} \int_{0}^{t}\left\|\mathbf{G}_{\epsilon}^{(1)}(s)\right\|_{\mathcal{H}_{1, \epsilon}}^{2} d s=\left\|\widehat{\mathbf{G}}_{\eta}\right\|_{L^{2}\left(\mathbb{T}_{\epsilon} \times \mathbb{T}\right)}^{2} \\
+2 \operatorname{Im}\left\{\int _ { 0 } ^ { t } d s \int _ { \mathbb { T } _ { \epsilon } \times \mathbb { T } } \left\{\left[\omega^{\prime}\left(k+\frac{\epsilon \eta}{2}\right)+\omega^{\prime}\left(k-\frac{\epsilon \eta}{2}\right)\right] \widehat{G}_{\epsilon,+}^{\mathrm{un}}(s, \eta, k)\left[\widehat{G}_{\epsilon, \eta,+}(s, \eta, k)\right]^{\star}\right.\right. \\
\left.\left.+\left[\omega^{\prime}\left(k+\frac{\epsilon \eta}{2}\right)-\omega^{\prime}\left(k-\frac{\epsilon \eta}{2}\right)\right] \widehat{H}_{\epsilon,+}^{\mathrm{un}}(s, \eta, k)\left[\widehat{H}_{\epsilon, \eta,+}(s, \eta, k)\right]^{\star} d \eta d k\right\}\right\} \\
-2 \epsilon \gamma_{0} \int_{0}^{t} d s \int_{\mathbb{T}_{\epsilon} \times \mathbb{T}}\left[R^{\prime}(k+\epsilon \eta / 2)-R^{\prime}(k-\epsilon \eta / 2)\right] \\
\quad \times \operatorname{Re}\left\{\widehat{G}_{\epsilon,+}(s, \eta, k)\left[\widehat{G}_{\epsilon, \eta,+}^{\mathrm{un}}(s, \eta, k)\right]^{\star}+\widehat{H}_{\epsilon,+}^{\mathrm{un}}(s, \eta, k)\left[\widehat{H}_{\epsilon, \eta,+}(s, \eta, k)\right]^{\star}\right\} d \eta d k \\
-4 \epsilon \gamma_{0} \int_{0}^{t} d s \int_{\mathbb{T}_{\epsilon} \times \mathbb{T}} R^{\prime}\left(k-\frac{\epsilon \eta}{2}\right) \operatorname{Re}\left\{\widehat{H}_{\epsilon,+}(s, \eta, k)\left[\widehat{G}_{\epsilon, \eta,+}(s, \eta, k)\right]^{\star}+\widehat{G}_{\epsilon,+}^{\mathrm{un}}(s, \eta, k)\left[\widehat{H}_{\epsilon, \eta,+}(s, \eta, k)\right]^{\star}\right\} d \eta d k .
\end{aligned}
$$

From (5.47) and (5.51) it follows directly that for any $\delta>0$ there exists a constant $C>0$ such that

$$
\begin{aligned}
& \left\|\widehat{\mathbf{G}}_{\epsilon, \eta}(t)\right\|_{L^{2}\left(\mathbb{T}_{\epsilon} \times \mathbb{T}\right)}^{2}+8 \gamma_{0} \int_{0}^{t}\left\|\mathbf{G}_{\epsilon}^{(1)}(s)\right\|_{\mathcal{H}_{0, \epsilon}}^{2} d s+4 \gamma_{1} \int_{0}^{t}\left\|\mathbf{G}_{\epsilon}^{(1)}(s)\right\|_{\mathcal{H}_{1, \epsilon}}^{2} d s \\
& \leq\left\|\widehat{\mathbf{G}}_{\eta}\right\|_{L^{2}\left(\mathbb{T}_{\epsilon} \times \mathbb{T}\right)}^{2}+\delta \int_{0}^{t}\left\|\widehat{\mathbf{G}}_{\epsilon, \eta}(s)\right\|_{L^{2}\left(\mathbb{T}_{\epsilon} \times \mathbb{T}\right)}^{2} d s+C t, \quad t \geq 0, \epsilon \in(0,1], t \geq 0 .
\end{aligned}
$$

Therefore, for any $\lambda_{0}>0$ we can find $C>0$ such that

$$
\left\|\partial_{\eta} \widehat{\mathbf{f}}(\lambda)\right\|_{L^{2}\left(\mathbb{T}_{\epsilon} \times \mathbb{T}\right)}^{2} \leq \frac{C}{\operatorname{Re} \lambda-\lambda_{0}}, \quad \operatorname{Re} \lambda>\lambda_{0}, \epsilon \in(0,1]
$$

and the conclusion of Lemma 5.8 follows.

\subsubsection{Proof of Lemma 5.9}

We show that for any $\rho>0$ there exists $M>0$ such that

$$
\limsup _{\epsilon \rightarrow 0+} \mathcal{I}_{\epsilon}(M)<\rho,
$$

where

$$
\begin{aligned}
& \mathcal{I}_{\epsilon}(M):=\int_{\left[\eta \in \mathbb{T}_{\epsilon},|\eta|>M\right]} d \eta \int_{\mathbb{T}^{2}} d k d k^{\prime} \mid \mathfrak{s}\left(k-\frac{\epsilon \eta}{2}\right) \mathfrak{s}\left(k+\frac{\epsilon \eta}{2}\right) \mathfrak{s}\left(k^{\prime}-\frac{\epsilon \eta}{2}\right) \mathfrak{s}\left(k^{\prime}+\frac{\epsilon \eta}{2}\right) \\
& \times \mathfrak{s}\left(k+k^{\prime}-\epsilon \eta\right) \mathfrak{s}\left(k+k^{\prime}+\epsilon \eta\right) \widehat{w}_{\epsilon,+}(\lambda, \eta, k) \widehat{g}_{\epsilon,+}^{\star}\left(\lambda, \eta, k^{\prime}\right) \mid .
\end{aligned}
$$


The proof in the case of the remaining terms appearing in the expression (5.43) carries out in a similar fashion. We shall also consider the case of an optical dispersion relation, that is somewhat more involved than the accoustic one, as then the dispersion relation has two critical points.

Recalling well known trigonometric identities we can write

$$
\mathfrak{s}\left(k+k^{\prime}-\epsilon \eta\right) \mathfrak{s}\left(k+k^{\prime}+\epsilon \eta\right)=\sum_{\iota_{1}, \iota_{2}=0,1} \mathfrak{s}_{\iota_{1}}\left(k^{\prime}-\frac{\epsilon \eta}{2}\right) \mathfrak{s}_{1-\iota_{1}}\left(k-\frac{\epsilon \eta}{2}\right) \mathfrak{s}_{\iota_{2}}\left(k^{\prime}+\frac{\epsilon \eta}{2}\right) \mathfrak{s}_{1-\iota_{2}}\left(k+\frac{\epsilon \eta}{2}\right) .
$$

Here $\mathfrak{s}_{0}(k):=\mathfrak{c}(k)$ and $\mathfrak{s}_{1}(k):=\mathfrak{s}(k)$. Correspondingly, expression (5.55) can be rewritten in the form $\sum_{\iota_{1}, \iota_{2} \in\{0,1\}} \mathcal{I}_{\iota_{1}, \iota_{2}}$. The analysis of each term is similar, so we only deal with $\iota_{1}=\iota_{2}=1$. The respective expression is of the form

$$
\begin{aligned}
& \mathcal{I}_{1,1}=\int_{\left[\eta \in \mathbb{T}_{\epsilon},|\eta|>M\right]} d \eta \int_{\mathbb{T}^{2}} d k d k^{\prime} \mid \mathfrak{s}(k-\epsilon \eta / 2) \mathfrak{c}(k-\epsilon \eta / 2) \mathfrak{s}(k+\epsilon \eta / 2) \mathfrak{c}(k+\epsilon \eta / 2) \widehat{w}_{\epsilon,+}(\lambda, \eta, k) \\
& \times \mathfrak{s}^{2}\left(k^{\prime}-\frac{\epsilon \eta}{2}\right) \mathfrak{s}^{2}\left(k^{\prime}+\frac{\epsilon \eta}{2}\right) \widehat{g}_{\epsilon,+}^{\star}\left(\lambda, \eta, k^{\prime}\right) \mid .
\end{aligned}
$$

We partition the domain of integration in (5.56) into two sets $T_{1}$ and $T_{2}$. To $T_{1}$ belong all those $\left(\eta, k, k^{\prime}\right)$, for which either

$$
\left|k \pm \frac{\epsilon \eta}{2}\right| \leq \delta, \quad \text { or } \quad 1 / 2-\delta \leq\left|k \pm \frac{\epsilon \eta}{2}\right| \leq 1 / 2
$$

while to $T_{2}$ belong all other $\left(\eta, k, k^{\prime}\right)$-s. Parameter $\delta \in(0,1 / 2)$ is to be chosen later on. We can write then $\mathcal{I}_{1,1}=\mathcal{I}_{1,1}^{1}+\mathcal{I}_{1,1}^{2}$, where $\mathcal{I}_{1,1}^{i}$ correspond to integration over $T_{i}, i=1,2$. We can write

$$
\mathcal{I}_{1,1}^{1} \preceq \delta \int_{\mathbb{T}_{\epsilon}} d \eta \int_{\mathbb{T}^{2}} d k d k^{\prime}\left|\widehat{w}_{\epsilon,+}(\lambda, \eta, k) \widehat{g}_{\epsilon,+}^{\star}\left(\lambda, \eta, k^{\prime}\right)\right| \preceq \delta,
$$

for $\epsilon \in(0,1]$, by virtue of (5.48) and (5.19).

Taking the Laplace transforms of both sides of (4.19) we obtain in particular that

$$
\widehat{w}_{\epsilon,+}(\lambda, \eta, k)=\left(\lambda+2 \gamma_{0} \bar{R}(k, \epsilon \eta)+i \delta_{\epsilon} \omega(k, \eta)\right)^{-1} D_{\epsilon}(\lambda, \eta, k),
$$

where

$$
\begin{aligned}
& D_{\epsilon}(\lambda, \eta, k):=\widehat{W}_{\epsilon,+}(\eta, k)-\frac{\gamma_{1}}{2}\left\{\mathfrak{d}_{\epsilon}\left(\lambda, k-\frac{\epsilon \eta}{2}\right)+\mathfrak{d}_{\epsilon}^{\star}\left(\lambda, k+\frac{\epsilon \eta}{2}\right)\right\} \\
& +\frac{\gamma_{0}}{2}\left(\mathcal{L}_{\epsilon \eta}^{+} \widehat{y}_{\epsilon,-}(\lambda, \eta, k)+\mathcal{L}_{-\epsilon \eta}^{+} \widehat{y}_{\epsilon,+}(\lambda, \eta, k)\right)-2 \gamma_{0} \mathcal{R}_{\epsilon \eta} \widehat{w}_{\epsilon,+}(\lambda, \eta, k) .
\end{aligned}
$$

Hence,

$$
\begin{aligned}
& \mathcal{I}_{1,1}^{2}=\int_{T_{2}} d \eta d k d k^{\prime} \mid \frac{\mathfrak{s}(k-\epsilon \eta / 2) \mathfrak{c}(k-\epsilon \eta / 2) \mathfrak{s}(k+\epsilon \eta / 2) \mathfrak{c}(k+\epsilon \eta / 2) d_{\epsilon}(\lambda, \eta, k)}{\lambda+2 \gamma_{0} \bar{R}(k, \epsilon \eta)+i \delta_{\epsilon} \omega(k, \eta)} \\
& \times \mathfrak{s}^{2}\left(k^{\prime}-\frac{\epsilon \eta}{2}\right) \mathfrak{s}^{2}\left(k^{\prime}+\frac{\epsilon \eta}{2}\right) \widehat{g}_{\epsilon,+}^{\star}\left(\lambda, \eta, k^{\prime}\right) \mid .
\end{aligned}
$$


Thanks to (5.19) and (5.34) there exists $\lambda_{0}$ such that

$$
d_{*}:=\sup _{\epsilon \in(0,1], \eta \in \mathbb{T}_{\epsilon}}\left\|D_{\epsilon}(\lambda, \eta, \cdot)\right\|_{L^{2}(\mathbb{T})}<+\infty, \quad \text { for } \operatorname{Re} \lambda>\lambda_{0} .
$$

Since $\omega^{\prime}(k) \neq 0$, except for $k=0,1 / 2$ we can find $c_{*}(\delta)>0$ such that

$$
\left|\lambda+2 \gamma_{0} \bar{R}(k, \epsilon \eta)+i \delta_{\epsilon} \omega(k, \eta)\right| \geq \lambda+c_{*}(\delta)|\eta| \quad \text { for }(\eta, k) \text { such that (5.57) does not hold. }
$$

Therefore

$$
\begin{aligned}
& \mathcal{I}_{1,1}^{2} \leq \int_{\left[\eta \in \mathbb{T}_{\epsilon},|\eta|>M\right]} d \eta \int_{\mathbb{T}^{2}} \frac{\left|D_{\epsilon}(\lambda, \eta, k) \widehat{g}_{\epsilon,+}^{\star}\left(\lambda, \eta, k^{\prime}\right)\right|}{\lambda+c_{*}(\delta)|\eta|} d k d k^{\prime} \\
& \leq\left\{\int_{[|\eta|>M]} \frac{d \eta}{\left(\lambda+c_{*}(\delta)|\eta|\right)^{2}} \sup _{\epsilon \in(0,1], \eta^{\prime} \in \mathbb{T}_{\epsilon}} \int_{\mathbb{T}}\left|D_{\epsilon}\left(\lambda, \eta^{\prime}, k\right)\right|^{2} d k\right\}^{1 / 2}\left\|g_{\epsilon,+}(\lambda)\right\|_{\mathcal{L}_{2, \epsilon}} .
\end{aligned}
$$

In light of (5.48) for any $\rho>0$ we can choose a sufficiently large $M$ so that $\lim \sup _{\epsilon \rightarrow 0+} \mathcal{I}_{1,1}^{2} \leq \rho / 2$. Adjusting suitably $\delta>0$, cf (5.58), we have also $\lim \sup _{\epsilon \rightarrow 0+} \mathcal{I}_{1,1}^{1} \leq \rho / 2$. Combining these two estimates we conclude that there exists $\lambda_{0}$ such that for any $\rho>0$ we can find $M>0$ for which

$$
\limsup _{\epsilon \rightarrow 0+} \mathcal{I}_{1,1}<\rho \text { for all } \operatorname{Re} \lambda>\lambda_{0}
$$

and the conclusion of the lemma follows.

\section{The case of arbitrary thermostat temperature $T$}

Setting the thermostat temperature at $T$ leads to the following dynamics of the Wigner functions $(\operatorname{cf}(4.19))$

$$
\frac{d}{d t} \widehat{\mathbf{W}}_{\epsilon}(t, \eta, k)=\left(\gamma_{0} \widehat{\mathfrak{L}}_{\epsilon \eta}+\widehat{\mathfrak{H}}_{\epsilon}+\gamma_{1} \widehat{\mathfrak{T}}_{\epsilon}\right) \widehat{\mathbf{W}}_{\epsilon}(t, \eta, k)+\frac{\gamma_{1} T}{\epsilon} \mathbf{D}
$$

Suppose that $\chi \in C_{c}^{\infty}(\mathbb{R})$ is an arbitrary real valued, even function satisfying (2.47). Then $\widehat{\chi} \in \mathcal{S}(\mathbb{R})$ and let $\hat{\chi}_{\epsilon} \in C^{\infty}\left(\mathbb{T}_{\epsilon}\right)$ be given by

$$
\widehat{\chi}_{\epsilon}(\eta):=\sum_{n \in \mathbb{Z}} \widehat{\chi}\left(\eta+\frac{2 n}{\epsilon}\right), \quad \eta \in \mathbb{T}_{\epsilon}
$$

Note that

$$
\int_{\mathbb{T}_{\epsilon}} \widehat{\chi}_{\epsilon}(\eta) d \eta=\int_{\mathbb{R}} \widehat{\chi}(n) d \eta=\chi(0)=1
$$

Define

$$
\widehat{\mathbf{V}}_{\epsilon}(t, \eta, k)=\left[\begin{array}{cc}
\widehat{V}_{\epsilon,+}(t, \eta, k) & \widehat{U}_{\epsilon,+}(t, \eta, k) \\
\widehat{U}_{\epsilon,-}(t, \eta, k) & \widehat{V}_{\epsilon,-}(t, \eta, k)
\end{array}\right]:=\widehat{\mathbf{W}}_{\epsilon}(t, \eta, k)-T \widehat{\chi}_{\epsilon}(\eta) \mathbf{I}_{2}
$$


where $\mathbf{I}_{2}$ is the $2 \times 2$ identity matrix, i.e.

$\widehat{V}_{\epsilon, \pm}(t, \eta, k):=\widehat{W}_{\epsilon, \pm}(t, \eta, k)-T \widehat{\chi}_{\epsilon}(\eta), \quad \widehat{U}_{\epsilon, \pm}(t, \eta, k):=\widehat{Y}_{\epsilon, \pm}(t, \eta, k), \quad t \geq 0,(\eta, k) \in \mathbb{T}_{\epsilon / 2} \times \mathbb{T}$

It satisfies

$$
\frac{d}{d t} \widehat{\mathbf{V}}_{\epsilon}(t, \eta, k)=\left(\gamma_{0} \widehat{\mathfrak{L}}_{\epsilon \eta}+\widehat{\mathfrak{H}}_{\epsilon}+\gamma_{1} \widehat{\mathfrak{T}}_{\epsilon}\right) \widehat{\mathbf{V}}_{\epsilon}(t, \eta, k)+\widehat{\mathbf{F}}_{\epsilon}(\eta, k),
$$

where

$$
\widehat{\mathbf{F}}_{\epsilon}(\eta, k):=-i \delta_{\epsilon} \omega(k ; \eta) T \widehat{\chi}_{\epsilon}(\eta) \mathbf{J}_{2}
$$

and

$$
\mathbf{J}_{2}:=\left[\begin{array}{cc}
1 & 0 \\
0 & -1
\end{array}\right] \text {. }
$$

The solution can be then written in the form, cf (4.20),

$$
\mathbf{V}_{\epsilon}(t)=\mathfrak{W}_{\epsilon}(t) \mathbf{V}_{\epsilon}(0)+\int_{0}^{t} \mathfrak{W}_{\epsilon}(s) \mathbf{F}_{\epsilon} d s
$$

Using the already proved part of Theorem 2.9 for $T=0$, we conclude that for any $\mathbf{G} \in$ $L^{1}([0,+\infty), \mathcal{A})$ we have

$$
\lim _{\epsilon \rightarrow 0+} \int_{0}^{+\infty}\left\langle\mathbf{V}_{\epsilon}(t), \mathbf{G}(t)\right\rangle d t=\int_{0}^{+\infty}\langle\mathbf{V}(t), \mathbf{G}(t)\rangle d t
$$

where

$$
\mathbf{V}(t, y, k)=\left[\begin{array}{cc}
V_{+}(t, y, k) & 0 \\
0 & V_{+}(t, y,-k)
\end{array}\right]
$$

and

$$
V_{+}(t, y, k):=\mathfrak{W}(t) V_{0,+}(y, k)-\int_{0}^{t} \mathfrak{W}(s) F(y, k) d s .
$$

Here $F$ is given by (2.49) and $V_{0,+}(y, k):=W_{0,+}(y, k)-T \chi(y)$. This ends the proof of Theorem 2.9 for an arbitrary $T \geq 0$.

\section{A Proof of Proposition 2.5}

Using formula (2.40) we can see that $\mathfrak{W}_{t}^{\text {un }}\left(\mathcal{C}_{0}^{\prime}\right) \subset \mathcal{C}_{0}^{\prime}, t \geq 0$. In addition (see Section 2.6.2) $\left(\mathfrak{W}_{t}^{\text {un }}\right)_{t \geq 0}$, given by (2.40), is a $C_{0}$-semigroup of contractions on $L^{2}(\mathbb{R} \times \mathbb{T})$. According to Section $\mathrm{A}$, of the Appendix of [14] the semigroup $\left(\mathfrak{W}_{t}\right)_{t \geq 0}$ is defined by the Duhamel series that corresponds to the equation (2.46). Since $\mathcal{R}$ is a bounded operator on $L^{2}(\mathbb{R} \times \mathbb{T})$ and $\left(\mathfrak{W}_{t}^{\text {un }}\right)_{t \geq 0}$ is a semigroups of contractions, the semigroup defined by the series is a $C_{0}$-semigroup of bounded operators on $L^{2}(\mathbb{R} \times$ 
$\mathbb{T})$. From here we conclude also that $\mathcal{C}_{0}^{\prime}$ has to be invariant under $\left(\mathfrak{W}_{t}\right)_{t \geq 0}$. For $W_{0} \in \mathcal{C}_{0}^{\prime}$ we conclude by a direct calculation that $W(t, y, k):=\mathfrak{W}_{t}\left(W_{0}\right)$ satisfies the following identity

$$
\begin{aligned}
& \frac{1}{2} \frac{d}{d t}\|W(t)\|_{L^{2}(\mathbb{R} \times \mathbb{T})}^{2}=-\gamma_{0} \int_{\mathbb{R} \times \mathbb{T}^{2}} R\left(k, k^{\prime}\right)\left[W(t, y, k)-W\left(t, y, k^{\prime}\right)\right]^{2} d y d k d k^{\prime} \\
& -\frac{1}{2} \int_{\mathbb{T}} \bar{\omega}^{\prime}(k)\left[W\left(t, 0^{-}, k\right)^{2}-W\left(t, 0^{+}, k\right)^{2}\right] d k, \quad t \geq 0 .
\end{aligned}
$$

Taking into account (2.35) and (2.36) we obtain

$$
\begin{aligned}
& \int_{\mathbb{T}} \bar{\omega}^{\prime}(k)\left\{\left[W\left(t, 0^{-}, k\right)\right]^{2}-\left[W\left(t, 0^{+}, k\right)\right]^{2}\right\} d k \\
& =\int_{\mathbb{T}_{+}} \bar{\omega}^{\prime}(k)\left\{\left[W\left(t, 0^{-}, k\right)\right]^{2}-\left[p_{-}(k) W\left(t, 0^{+},-k\right)+p_{+}(k) W\left(t, 0^{-}, k\right)\right]^{2}\right\} d k \\
& +\int_{\mathbb{T}_{-}} \bar{\omega}^{\prime}(k)\left\{\left[p_{-}(k) W\left(t, 0^{-},-k\right)+p_{+}(k) W\left(t, 0^{+}, k\right)\right]^{2}-\left[W\left(t, 0^{+}, k\right)\right]^{2}\right\} d k .
\end{aligned}
$$

After straightforward calculations (recall that coefficients $p_{ \pm}(k)$ are even, while $\bar{\omega}^{\prime}(k)$ is odd) we conclude that the right hand side equals

$$
\begin{gathered}
\int_{\mathbb{T}_{+}} \bar{\omega}^{\prime}(k)\left\{\left(W\left(t, 0^{-}, k\right)^{2}+W\left(t, 0^{+},-k\right)^{2}\right)\left(1-p_{+}^{2}(k)-p_{-}^{2}(k)\right)\right. \\
\left.-4 p_{-}(k) p_{+}(k) W\left(t, 0^{+},-k\right) W\left(t, 0^{-}, k\right)\right\} d k .
\end{gathered}
$$

Since $p_{+}(k)+p_{-}(k) \leq 1$ we have $1-p_{+}^{2}(k)-p_{-}^{2}(k) \geq 0$. In addition,

$$
\begin{aligned}
& \operatorname{det}\left[\begin{array}{cc}
1-p_{+}^{2}(k)-p_{-}^{2}(k) & -2 p_{-}(k) p_{+}(k) \\
-2 p_{-}(k) p_{+}(k) & 1-p_{+}^{2}(k)-p_{-}^{2}(k)
\end{array}\right] \\
& =\left[1-\left(p_{+}(k)+p_{-}(k)\right)^{2}\right]\left[1-\left(p_{+}(k)-p_{-}(k)\right)^{2}\right] \geq 0 .
\end{aligned}
$$

Using (2.31) we conclude that the quadratic form

$$
(x, y) \mapsto\left(1-p_{+}^{2}(k)-p_{-}^{2}(k)\right)\left(x^{2}+y^{2}\right)-4 p_{-}(k) p_{+}(k) x y
$$

is non-negative definite ( since $\left.p_{+}(k)+p_{-}(k) \leq 1\right)$. Hence, in particular

$$
\frac{d}{d t}\|W(t)\|_{L^{2}(\mathbb{R} \times \mathbb{T})}^{2} \leq 0, \quad t \geq 0
$$

which in turn proves that $\left(\mathfrak{W}_{t}\right)_{t \geq 0}$ is a semigroup of contractions. $\square$ 


\section{B Outline of the proof of Theorem 5.6}

The proof of Theorem 5.6, for the most part, follows closely the argument contained in [13]. We shall present here its outline, invoking the relevant parts of [13] and focus on the necessary modifications. We start with the following.

Proposition B.1 Suppose that the initial data satisfies the assumptions of Theorem 2.9. Then,

$$
\lim _{\epsilon \rightarrow 0+} \int_{\mathbb{R} \times \mathbb{T}} \widehat{y}_{\epsilon, \iota}^{\mathrm{un}}(\lambda, \eta, k) \widehat{G}^{\star}(\eta, k) d \eta d k=0, \quad \iota= \pm
$$

for any $\operatorname{Re} \lambda>0$ and $G \in \mathcal{A}$.

The proof of the proposition follows the argument presented in Section 5.5.1, with the simplification consisting in the fact that the equation of $\widehat{y}_{\epsilon,+}^{\mathrm{un}}(\lambda, \eta, k)$ corresponding to (5.33) does not contain the scattering terms involving the operator $\mathcal{R}_{\epsilon \eta}$.

Next, we show that

$$
\lim _{\epsilon \rightarrow 0+} \int_{\mathbb{R} \times \mathbb{T}} \widehat{w}_{\epsilon,+}^{\mathrm{un}}(\lambda, \eta, k) \widehat{G}^{\star}(\eta, k) d \eta d k=\int_{\mathbb{R} \times \mathbb{T}} \widehat{w}_{+}^{\mathrm{un}}(\lambda, \eta, k) \widehat{G}^{\star}(\eta, k) d \eta d k
$$

where $w_{+}^{\text {un }}(\lambda)$ is given by (5.24) and $G \in \mathcal{A}_{c}$.

We let $\operatorname{supp} \hat{G} \subset[-K, K] \times \mathbb{T}$. Taking the Laplace transforms of both sides of (5.1) we obtain in particular the equation

$$
\begin{aligned}
& \left(\lambda+2 \gamma_{0} \bar{R}(k, \epsilon \eta)+i \delta_{\epsilon} \omega(k, \eta)\right) \widehat{w}_{\epsilon,+}^{\mathrm{un}}(\lambda, \eta, k)+\gamma_{0} R\left(k-\frac{\epsilon \eta}{2}\right) \widehat{y}_{\epsilon,+}^{\mathrm{un}}(\lambda, \eta, k)+\gamma_{0} R\left(k+\frac{\epsilon \eta}{2}\right) \widehat{y}_{\epsilon,-}^{\mathrm{un}}(\lambda, \eta, k) \\
& =\widehat{W}_{\epsilon,+}(0, \eta, k)-\frac{\gamma_{1}}{2}\left\{\mathfrak{d}_{\epsilon}^{\mathrm{un}}\left(\lambda, k-\frac{\epsilon \eta}{2}\right)+\left(\mathfrak{d}_{\epsilon}^{\mathrm{un}}\right)^{\star}\left(\lambda, k+\frac{\epsilon \eta}{2}\right)\right\}
\end{aligned}
$$

Here $(\operatorname{cf}(\underline{5.5}))$

$$
\mathfrak{d}_{\epsilon}^{\mathrm{un}}(\lambda, k):=\int_{0}^{+\infty} e^{-\lambda t} d_{\epsilon}(t, k) d s=\frac{1}{2} \int_{\mathbb{T}_{\epsilon}}\left[\widehat{y}_{\epsilon,+}^{\mathrm{un}}\left(\lambda, \eta, k-\frac{\epsilon \eta}{2}\right)-\widehat{w}_{\epsilon,+}^{\mathrm{un}}\left(\lambda, \eta, k-\frac{\epsilon \eta}{2}\right)\right] d \eta .
$$

Thanks to (5.7) we conclude

$$
\left\|\mathfrak{d}_{\epsilon}^{\text {un }}(\lambda)\right\|_{L^{2}(\mathbb{T})} \leq \frac{\left\|\mathbf{W}_{\epsilon}(0)\right\|_{\mathcal{L}_{2, \epsilon}}}{\left(2^{5} \gamma_{1} \operatorname{Re} \lambda\right)^{1 / 2}}, \quad \epsilon \in(0,1], \operatorname{Re} \lambda>0
$$

Suppose that $\epsilon_{n} \rightarrow 0$ as a sequence that corresponds to a *-weakly convergent in $\mathcal{A}^{\prime}$ subsequence $\left(w_{\epsilon_{n},+}^{\mathrm{un}}(\lambda, \cdot)\right)$ and $[-K, K] \subset\left[-\epsilon_{n}^{-1}, \epsilon_{n}^{-1}\right], n \geq 1$. By virtue of Proposition 5.1 families $\left(\widehat{w}_{\epsilon_{n},+}^{\mathrm{un}}(\lambda) \chi_{K}\right)$ 
(see (5.31) $)$ and $\left(\mathfrak{d}_{\epsilon_{n}} \chi_{K}\right)$ are ${ }^{\star}$-weakly compact in $L^{2}(\mathbb{R} \times \mathbb{T})$. Let $\widehat{w}_{+}^{\text {un }}(\lambda, \cdot)$ and $\mathfrak{d}(\lambda, \cdot)$ be their respective limits. Multiplying equation $(\underline{\mathrm{B} .3})$ by $\chi_{K}$ and letting $\epsilon \rightarrow 0+$ we conclude that

$$
\left(\lambda+2 \gamma_{0} R(k)+i \omega^{\prime}(k) \eta\right) \widehat{w}_{+}^{\text {un }}=\widehat{W}_{0}(\eta, k)-\gamma_{1} \operatorname{Re} \mathfrak{d}^{\text {un }}(\lambda, k)
$$

where

$$
\int_{\mathbb{T}} \operatorname{Re} \mathfrak{d}^{\text {un }}(\lambda, k) \widehat{G}^{\star}(k) d k=\lim _{\epsilon \rightarrow 0+} \int_{\mathbb{T}} \operatorname{Re} \mathfrak{d}_{\epsilon}^{\text {un }}(\lambda, k) \widehat{G}^{\star}(k) d k, \quad \widehat{G} \in C^{\infty}(\mathbb{T}) .
$$

The conclusion of Theorem 5.6 follows from.

Theorem B.2 For any $\lambda>0$ we have

$$
\operatorname{Re} \mathfrak{d}(\lambda, k)=\left(1-p_{+}(k)\right) \int_{\mathbb{R}} \frac{\widehat{W}_{0}(\eta, k) d \eta d k}{\lambda+2 \gamma_{0} R(k)+i \omega^{\prime}(k) \eta}-p_{-}(k) \int_{\mathbb{R}} \frac{\widehat{W}_{0}(\eta,-k) d \eta}{\lambda+2 \gamma_{0} R(k)-i \omega^{\prime}(k) \eta}, \quad k \in \mathbb{T} .
$$

\section{B.1 Proof of Theorem B.2}

To simplify somewhat our presentation we shall assume that

$$
\langle\hat{\psi}(k) \hat{\psi}(\ell)\rangle_{\mu_{\epsilon}}=0, \quad k, \ell \in \mathbb{T} .
$$

The result remains valid without this hypothesis, although the calculations become more extensive. Assumption (B.8) results in the condition

$$
\widehat{Y}_{\epsilon, \pm}(0, \eta, k)=0, \quad(\eta, k) \in \mathbb{R} \times \mathbb{T} .
$$

Using (3.24) we may write

$$
\mathfrak{d}_{\epsilon}^{\text {un }}(\lambda, k)=\mathfrak{d}_{\epsilon}^{1}(\lambda, k)+\mathfrak{d}_{\epsilon}^{2}(\lambda, k)
$$

Here, $\mathfrak{d}_{\epsilon}^{j}(\lambda, k), j=1,2$ are the respective Laplace transforms of

$$
\begin{gathered}
I_{\epsilon}(t, k):=i \int_{0}^{t}\left\langle p_{0}^{0}(t-s) \mathfrak{e}_{1} \cdot e_{\Omega_{\epsilon}}(k, t) \hat{\Psi}(k)\right\rangle_{\mu_{\epsilon}} g_{\epsilon}(d s), \\
I_{\epsilon}(t, k):=-\gamma_{1} \int_{0}^{t} g_{\epsilon}\left(d s^{\prime}\right) \int_{0}^{t} \Theta(t-s, k)\left\langle p_{0}^{0}(s) p_{0}^{0}\left(t-s^{\prime}\right)\right\rangle_{\mu_{\epsilon}} d s
\end{gathered}
$$

Here $e_{\Omega_{\epsilon}}(k, t)$ is given by (3.9) and

$$
p_{0}^{0}(t)=\frac{1}{2 i} \int_{\mathbb{T}} e_{\Omega_{\epsilon}}(k, t) \hat{\Psi}(k) \cdot \mathfrak{f} d k, \quad \Theta(t, k):=\int_{0}^{t} \mathfrak{e}_{1} \cdot e_{\Omega_{\epsilon}}(k, t-\tau) \mathfrak{f} g_{\epsilon}(d \tau) .
$$

We introduce

$$
\mathfrak{L}_{\text {scat }}^{\epsilon}(\lambda):=-\gamma_{1} \int_{\mathbb{T}} \hat{G}^{*}(k) \operatorname{Re} \mathfrak{d}_{\epsilon}(\lambda, k) d k=\sum_{j=1}^{2} \mathfrak{L}_{\text {scat }, j}^{\epsilon}(\lambda)
$$

with

$$
\mathfrak{L}_{\text {scat }, j}^{\epsilon}(\lambda):=-\gamma_{1} \int_{\mathbb{T}} \hat{G}^{*}(k) \operatorname{Re} \mathfrak{d}_{\epsilon}^{j}(\lambda, k) d k, \quad j=1,2
$$




\section{B.1.1 The limit of $\mathfrak{L}_{\text {scat }, 1}^{\epsilon}(\lambda)$}

We will show the following.

Lemma B.3 For any test function $\widehat{G} \in C^{\infty}(\mathbb{T})$ and $\lambda>0$ we have

$$
\lim _{\epsilon \rightarrow 0+} \mathfrak{L}_{\text {scat }, 1}^{\epsilon}(\lambda)=-\gamma_{1} \int_{\mathbb{T}} \operatorname{Re}[\nu(k)] \widehat{G}^{*}(k) d k \int_{\mathbb{R}} \frac{\widehat{W}_{0}(\eta, k) d \eta}{\lambda+2 \gamma_{0} R(k)+i \omega^{\prime}(k) \eta} d \eta^{\prime} .
$$

Proof. From (B.11) and (B.8) we get

$$
\begin{gathered}
I_{\epsilon}(t, k)=\frac{1}{2}\left\{e_{\Omega_{\epsilon}}^{1,1}(k, t) \int_{0}^{t} g_{\epsilon}(d s) \int_{\mathbb{T}} e_{\Omega_{\epsilon}}(\ell, t-s) \mathfrak{e}_{1} \cdot \mathfrak{f}\left\langle\hat{\psi}^{\star}(k) \hat{\psi}(\ell)\right\rangle_{\mu_{\epsilon}}\right. \\
\left.+e_{\Omega_{\epsilon}}^{1,2}(k, t) \int_{0}^{t} g_{\epsilon}(d s) \int_{\mathbb{T}} e_{\Omega_{\epsilon}}(\ell, t-s) \mathfrak{e}_{1} \cdot \mathfrak{f}\left\langle\hat{\psi}(-k) \hat{\psi}^{\star}(-\ell)\right\rangle_{\mu_{\epsilon}}\right\} d \ell .
\end{gathered}
$$

Using formula (3.9) we can write

$$
\begin{aligned}
& e_{\Omega_{\epsilon}}^{1,1}(k, t)=e^{\lambda_{+}(k) t}+\epsilon \sum_{\iota= \pm} r_{\iota, \epsilon}^{1}(k) e^{\lambda_{\iota} t}, \quad e_{\Omega_{\epsilon}}^{1,2}(k, t)=\epsilon \sum_{\iota= \pm} r_{\iota, \epsilon}^{2}(k) e^{\lambda_{\iota} t} \\
& e_{\Omega_{\epsilon}}(\ell, t-s) \mathfrak{e}_{1} \cdot \mathfrak{f}=e^{\lambda_{-}(\ell)(t-s)}+\epsilon \sum_{\iota= \pm} r_{\iota, \epsilon}^{3}(\ell) e^{\lambda_{\iota}(\ell)(t-s)}
\end{aligned}
$$

where

$$
\sup _{k \in \mathbb{T}, \epsilon \in(0,1]}\left|r_{\iota, \epsilon}^{j}(k)\right|=r_{\iota, *}^{j}<+\infty, \quad, \iota \in\{-,+\}, j=1,2,3 .
$$

The following result allows us to replace the entries of $e_{\Omega_{\epsilon}}(k, t)$ by the leading terms appearing in (B.16). Define

$$
\tilde{I}_{\epsilon}^{\iota_{1}, \iota_{2}}(t, k):=\epsilon r_{1, \epsilon}(k) e^{\lambda_{\iota_{1}}(k) t} \int_{0}^{t} g_{\epsilon}(d s) \int_{\mathbb{T}} r_{2, \epsilon}(\ell) e^{\lambda_{\iota_{2}}(\ell)(t-s)}\left\langle\hat{\psi}^{\star}(k) \hat{\psi}(\ell)\right\rangle_{\mu_{\epsilon}} d \ell
$$

and

$$
\tilde{I}_{\epsilon}^{\iota_{1}, \iota_{2}}(\lambda, k):=\int_{0}^{+\infty} e^{-\lambda t} \tilde{I}_{\epsilon}^{\iota_{1}, \iota_{2}}\left(\frac{t}{\epsilon}, k\right) d t
$$

Lemma B.4 Suppose that (2.12) holds,

$$
\sup _{k \in \mathbb{T}, \epsilon \in(0,1]}\left|r_{i, \epsilon}(k)\right|=r_{i, *}<+\infty, \quad i=1,2 .
$$

Then, for any $\iota_{1}, \iota_{2} \in\{-,+\}$ and $\lambda>0$ we have

$$
\left\|\tilde{I}_{\epsilon}^{\iota_{1}, \iota_{2}}(\lambda)\right\|_{L^{1}(\mathbb{T})} \preceq \epsilon^{1 / 2} \log \epsilon^{-1}, \quad \epsilon \in(0,1] .
$$


The proof of this lemma is presented in Section B.4.1

Using Lemma B.4 we conclude that

$$
\begin{aligned}
& \int_{\mathbb{T}} \mathfrak{d}_{\epsilon}^{1}(\lambda, k) \widehat{G}^{\star}(k) d k=\frac{\epsilon}{2} \int_{\mathbb{T}^{2}} \epsilon\left\langle\hat{\psi}^{\star}(k) \hat{\psi}(\ell)\right\rangle_{\mu_{\epsilon}} \widehat{G}^{\star}(k) d \ell d k \\
& \times \int_{0}^{+\infty} \exp \left\{-\lambda_{-}(\ell) s\right\} g_{\epsilon}(d s) \int_{s}^{+\infty} e^{-\lambda \epsilon t} \exp \left\{\left[\lambda_{+}(k)+\lambda_{-}(\ell)\right] t\right\} d t+O(\epsilon) \\
& =\frac{1}{2} \int_{\mathbb{T}^{2}} \epsilon\left\langle\hat{\psi}^{\star}(k) \hat{\psi}(\ell)\right\rangle_{\mu_{\epsilon}} \widehat{G}^{\star}(k) \tilde{g}_{\epsilon}\left(\epsilon\left(\lambda+\gamma_{0} R(k)\right)-i \omega_{\epsilon}(k)\right) d \ell d k \\
& \times\left\{\epsilon\left(\lambda+\gamma_{0} R(k)+\gamma_{0} R(\ell)\right)+i\left[\omega_{\epsilon}(\ell)-\omega_{\epsilon}(k)\right]\right\}^{-1}+O(\epsilon)
\end{aligned}
$$

Changing variables $k:=k^{\prime}-\epsilon \eta^{\prime} / 2, \ell:=k^{\prime}+\epsilon \eta^{\prime} / 2$ we can write (cf (3.17) and (3.6))

$$
\int_{\mathbb{T}} \mathfrak{d}_{\epsilon}^{1}(\lambda, k) \widehat{G}^{\star}(k) d k=\mathcal{I}_{\epsilon}+O(\epsilon),
$$

where

$$
\mathcal{I}_{\epsilon}:=\int_{T_{\epsilon}} \widehat{W}_{\epsilon,+}(0, \eta, k) \tilde{g}_{\epsilon}\left(\epsilon\left(\lambda+\gamma_{0} R(k-\epsilon \eta / 2)\right)-i \omega_{\epsilon}(k-\epsilon \eta / 2)\right) \Delta_{\epsilon}(k, \eta) d \eta d k .
$$

Here (cf (4.10) and (4.11)

$$
\Delta_{\epsilon}(k, \eta):=\left\{\lambda+2 \gamma_{0} \bar{R}(k, \epsilon \eta)+i \delta_{\epsilon} \omega_{\epsilon}(k ; \eta)\right\}^{-1} \widehat{G}^{\star}(k-\epsilon \eta / 2)
$$

and $T_{\epsilon}$ is the image of $\mathbb{T}^{2}$ under the inverse map $k^{\prime}:=(\ell+k) / 2, \eta^{\prime}:=(\ell-k) / \epsilon$.

We claim that in fact

$$
\lim _{\epsilon \rightarrow 0+}\left(\mathcal{I}_{\epsilon}-\mathcal{I}_{\epsilon}^{\prime}\right)=0,
$$

where the definition of the expression $\mathcal{I}_{\epsilon}^{\prime}$ differs from $\mathcal{I}_{\epsilon}$ only by replacing $\tilde{g}_{\epsilon}$ by $\tilde{g}$. Changing variable $k \mapsto k-\epsilon \eta / 2$ we can write

$$
\mathcal{I}_{\epsilon}-\mathcal{I}_{\epsilon}^{\prime}=\int_{T_{\epsilon}^{\prime}} \widehat{W}_{\epsilon,+}(0, \eta, k+\epsilon \eta / 2)\left(\tilde{g}_{\epsilon}-\tilde{g}\right)\left(\epsilon\left(\lambda+\gamma_{0} R(k)\right)-i \omega_{\epsilon}(k)\right) \Delta_{\epsilon}(k+\epsilon \eta / 2, \eta) d \eta d k .
$$

Here $T_{\epsilon}^{\prime}$ is the impage of $T_{\epsilon}$ under the change of variable. Equality (B.22) follows from Proposition 3.1 and the fact that the expression under the integral in the right hand side is bounded by an integrable function, see (2.51).

Next, thanks to Lemma 7.3 of [13], we have

$$
\lim _{\epsilon \rightarrow 0} \tilde{g}\left(\epsilon\left(\lambda+\gamma_{0} R(k-\epsilon \eta / 2)\right)-i \omega_{\epsilon}(k-\epsilon \eta / 2)\right) \Delta_{\epsilon}(k, \eta)=\frac{\nu(k) \widehat{G}^{\star}(k)}{\lambda+2 \gamma_{0} R(k)+i \omega^{\prime}(k) \eta}
$$

a.e. in $(\eta, k)$. Using bounds (2.51) and (3.18) we can argue, via the dominated convergence theorem, as in the proof of Lemma 5.1 of [13, that

$$
\lim _{\epsilon \rightarrow 0+} \mathcal{I}_{\epsilon}^{\prime}=\int_{\mathbb{R} \times \mathbb{T}} \frac{\widehat{W}_{0}(\eta, k) \nu(k) \widehat{G}^{*}(k) d \eta d k}{\lambda+2 \gamma_{0} R(k)+i \omega^{\prime}(k) \eta}
$$


and conclusion of Lemma B.3 follows.

Concerning the second term in the utmost right hand side of (B.12) we have the following.

Lemma B.5 For any $\lambda>0$ and $G \in C^{\infty}(\mathbb{T})$ we have

$$
\lim _{\epsilon \rightarrow 0} \mathfrak{L}_{s c a t, 2}^{\epsilon}(\lambda)=\frac{\gamma_{1}}{4} \sum_{\iota= \pm} \int_{\mathbb{T}} \frac{\hat{G}^{\star}(k) \delta(k) \widehat{W}_{0}(\eta, \iota k) d \eta d k}{\lambda+2 \gamma_{0} R(k)+\iota i \omega^{\prime}(k) \eta} .
$$

The proof of the lemma follows very closely the proof of Lemma 6.4 of [13]. We present its outline in Section B.3 below.

\section{B.2 The limit of $\mathfrak{L}_{\text {scat }}^{\epsilon}(\lambda)$}

Putting together the results of Lemmas B.3 and B.5, we see that

$$
\lim _{\epsilon \rightarrow 0} \mathfrak{L}_{\text {scat }}^{\epsilon}(\lambda)=\int_{\mathbb{T}}\left(\mathcal{W}_{t r}(k)+\mathcal{W}_{\text {ref }}(k)\right) \hat{G}^{*}(k) d k,
$$

with the transmission term

$$
\begin{aligned}
& \mathcal{W}_{t r}(k)=\frac{\gamma_{1}}{\left|\bar{\omega}^{\prime}(k)\right|}\left\{-\operatorname{Re}[\nu(k)]+\frac{g(k)}{4}\right\} \int_{\mathbb{R}} \frac{\widehat{W}_{0}(\eta, k) d \eta}{\lambda+2 \gamma_{0} R(k)+i \omega^{\prime}(k) \eta} \\
& =\left(p_{+}(k)-1\right) \int_{\mathbb{R}} \frac{\widehat{W}_{0}(\eta, k) d \eta}{\lambda+2 \gamma_{0} R(k)+i \omega^{\prime}(k) \eta} .
\end{aligned}
$$

We have used (2.30) in the last step. The reflection term $\mathcal{W}_{\text {ref }}(k)$ equals $(\operatorname{cf}(2.29))$

$$
\mathcal{W}_{r e f}(k)=\frac{\gamma_{1} \delta(k)}{4\left|\bar{\omega}^{\prime}(k)\right|} \int_{\mathbb{R}} \frac{\widehat{W}_{0}(\eta,-k) d \eta}{\lambda+2 \gamma_{0} R(k)-i \omega^{\prime}(k) \eta}=p_{-}(k) \int_{\mathbb{R}} \frac{\widehat{W}_{0}(\eta,-k) d \eta}{\lambda+2 \gamma_{0} R(k)-i \omega^{\prime}(k) \eta} .
$$

Combining the scattering terms in (B.25) $-($ B.27) we obtain (B.7). Thus, the proof of Theorem B.2 is reduced to showing Lemma B.5.

\section{B.3 Outline of the proof of Lemma B.5: the limit of $\mathfrak{L}_{s c a t, 2}^{\epsilon}(\lambda)$}

Let

$$
\begin{aligned}
& \widetilde{I I}_{\epsilon}^{\iota_{1}, \iota_{2}, \iota_{3}}(t, k):=\epsilon r_{1, \epsilon}(k) \int_{0}^{t} d s \int_{0}^{t} g_{\epsilon}\left(d s^{\prime}\right) \int_{0}^{s} g_{\epsilon}\left(d s_{1}\right) \\
& \times \int_{\mathbb{T}^{2}} r_{2, \epsilon}(\ell) r_{3, \epsilon}\left(\ell^{\prime}\right) e^{\lambda_{\iota_{2}}(\ell)\left(s-s_{1}\right)} e^{\lambda_{\iota_{3}}\left(\ell^{\prime}\right)\left(t-s^{\prime}\right)} e^{\lambda_{\iota_{1}}(k)(t-s)}\left\langle\hat{\psi}^{\star}(\ell) \hat{\psi}\left(\ell^{\prime}\right)\right\rangle_{\mu_{\epsilon}} d \ell d \ell^{\prime}
\end{aligned}
$$

and

$$
\widetilde{I I}_{\epsilon}^{\iota_{1}, \iota_{2}, \iota_{3}}(\lambda, k):=\int_{0}^{+\infty} e^{-\lambda t} \widetilde{I I}_{\epsilon}^{\iota_{1}, \iota_{2}, \iota_{3}}\left(\frac{t}{\epsilon}, k\right) d t .
$$

We start with the following. 
Lemma B.6 Suppose that condition (2.51) holds,

$$
\sup _{k \in \mathbb{T}, \epsilon \in(0,1]}\left|r_{i, \epsilon}(k)\right|=r_{i, *}<+\infty, \quad i=1,2,3 .
$$

Then, for any $\iota_{1}, \iota_{2}, \iota_{3} \in\{-,+\}$ and $\operatorname{Re} \lambda>0$ we have

$$
\lim _{\epsilon \rightarrow 0+}\left\|\widetilde{I I}_{\epsilon}^{\iota_{1}, \iota_{2}, \iota_{3}}(\lambda)\right\|_{L^{1}(\mathbb{T})}=0
$$

The proof of the lemma is presented in Section B.4.2 below.

Using Lemma B.6 we can write

$$
\mathfrak{L}_{\text {scat }, 2}^{\epsilon}(\lambda)=\overline{\mathfrak{L}}_{\text {scat }, 2}^{\epsilon}(\lambda)+o(1),
$$

as $\epsilon \rightarrow 0+$, where

$$
\overline{\mathfrak{L}}_{\text {scat }, 2}^{\epsilon}(\lambda):=-\gamma_{1} \int_{\mathbb{T}} \operatorname{Re} \overline{\mathfrak{d}}_{\epsilon}^{2}(\lambda, k) \hat{G}^{\star}(k) d k
$$

and

$$
\overline{\mathfrak{d}}_{\epsilon}^{2}(\lambda, k):=-\gamma_{1} \epsilon \int_{0}^{+\infty} e^{-\epsilon \lambda t} d t\left\langle\bar{p}_{0}^{0} \star g_{\epsilon}(t) \int_{0}^{t} \bar{p}_{0}^{0} \star g_{\epsilon}(s) d s\right\rangle_{\mu_{\epsilon}} e^{\lambda_{+}(k)(t-s)}
$$

Here

$$
\bar{p}_{0}^{0}(t):=\int_{\mathbb{T}} e^{-\epsilon \gamma_{0} R(k) t} \operatorname{Im}\left(\hat{\psi}(k) e^{-i \omega(k) t}\right) d k .
$$

Since $\bar{p}_{0}^{0} \star g_{\epsilon}$ is real valued we have

$$
\operatorname{Re} \overline{\mathfrak{d}}_{\epsilon}^{2}(\lambda, k):=-\gamma_{1} \epsilon \int_{0}^{+\infty} e^{-\epsilon \lambda t} d t\left\langle\bar{p}_{0}^{0} \star g_{\epsilon}(t) \int_{0}^{t} \bar{p}_{0}^{0} \star g_{\epsilon}(s) d s\right\rangle_{\mu_{\epsilon}} e^{-\gamma_{0} \epsilon R(k)(t-s)} \cos \left(\omega_{\epsilon}(k)(t-s)\right) .
$$

After rather lengthy, cut starightforward calculation, see Section B.4.3 below for details, we get

$$
2 \operatorname{Re} \overline{\mathfrak{d}}_{\epsilon}^{2}(\lambda, k)=R_{\epsilon}(\lambda, k)+\rho_{\epsilon}(\lambda, k)
$$

with

$$
\begin{aligned}
& R_{\epsilon}(\lambda, k):=-\frac{\gamma_{1}\left(\lambda+2 \gamma_{0} \epsilon R(k)\right)}{2^{3} \cdot \pi \epsilon^{2}} \int_{\mathbb{R}} \frac{d \xi}{\left(\lambda / 2+\gamma_{0} R(k)\right)^{2}+\xi^{2}} \int_{\mathbb{T}^{2}} d \ell d \ell^{\prime} \epsilon\left\langle\hat{\psi}(\ell) \hat{\psi}^{*}\left(\ell^{\prime}\right)\right\rangle_{\mu_{\epsilon}} \\
& \times \frac{\left|\tilde{g}_{\epsilon}\left(\lambda \epsilon / 2-i\left[\epsilon \xi+\omega_{\epsilon}(k)\right]\right)\right|^{2}}{\lambda / 2-i\left\{\xi+\epsilon^{-1}\left[\omega_{\epsilon}(k)-\omega_{\epsilon}(\ell)\right]\right\}} \times \frac{1}{\lambda / 2+i\left\{\xi+\epsilon^{-1}\left[\omega_{\epsilon}(k)-\omega_{\epsilon}\left(\ell^{\prime}\right)\right]\right\}}
\end{aligned}
$$


and

$$
\begin{aligned}
& \rho_{\epsilon}(\lambda, k):=-\frac{\epsilon \gamma_{1}\left(\lambda+2 \gamma_{0} \epsilon R(k)\right)}{2^{4} \cdot \pi} \int_{\mathbb{R}} \frac{d \xi}{\left(\lambda / 2+\gamma_{0} R(k)\right)^{2}+\xi^{2}} \int_{\mathbb{T}^{2}} d \ell d \ell^{\prime}\left\langle\hat{\psi}(\ell) \hat{\psi}^{*}\left(\ell^{\prime}\right)\right\rangle_{\mu_{\epsilon}} \\
& \times\left\{\frac{\tilde{g}\left(\lambda \epsilon / 2-i\left[\epsilon \xi+\omega_{\epsilon}(k)\right]\right)}{\epsilon \lambda / 2-i\left[\epsilon \xi+\omega_{\epsilon}(k)-\omega_{\epsilon}(\ell)\right]}\right. \\
& \times\left\{\frac{\tilde{g}\left(\lambda \epsilon / 2+i\left[\epsilon \xi+\omega_{\epsilon}(k)\right]\right)}{\epsilon \lambda / 2+i\left[\epsilon \xi+\omega_{\epsilon}(k)+\omega_{\epsilon}\left(\ell^{\prime}\right)\right]}+\frac{\left.\tilde{g}\left(\lambda \epsilon / 2+i\left[\epsilon \xi-\omega_{\epsilon}(k)\right]\right)\right)}{\epsilon \lambda / 2+i\left\{\epsilon \xi-\left[\omega_{\epsilon}(k)+\omega_{\epsilon}\left(\ell^{\prime}\right)\right]\right\}}\right\} \\
& +\frac{\tilde{g}\left(\lambda \epsilon / 2-i\left[\epsilon \xi-\omega_{\epsilon}(k)\right]\right)}{\epsilon \lambda / 2-i\left[\epsilon \xi+\omega_{\epsilon}(k)+\omega_{\epsilon}(\ell)\right]} \\
& \times\left\{\frac{\tilde{g}\left(\lambda \epsilon / 2+i\left[\epsilon \xi+\omega_{\epsilon}(k)\right]\right)}{\epsilon \lambda / 2+i\left[\epsilon \xi+\omega_{\epsilon}(k)+\omega_{\epsilon}\left(\ell^{\prime}\right)\right]}+\frac{\left.\tilde{g}\left(\lambda \epsilon / 2+i\left[\epsilon \xi-\omega_{\epsilon}(k)\right]\right)\right)}{\epsilon \lambda / 2+i\left\{\epsilon \xi-\left[\omega_{\epsilon}(k)+\omega_{\epsilon}\left(\ell^{\prime}\right)\right]\right\}}\right\} \\
& -\frac{\tilde{g}\left(\lambda \epsilon / 2-i\left[\epsilon \xi+\omega_{\epsilon}(k)\right]\right)}{\epsilon \lambda / 2-i\left[\epsilon \xi+\omega_{\epsilon}(k)-\omega_{\epsilon}(\ell)\right]} \cdot \frac{\left.\tilde{g}\left(\lambda \epsilon / 2+i\left[\epsilon \xi-\omega_{\epsilon}(k)\right]\right)\right)}{\epsilon \lambda / 2+i\left\{\epsilon \xi-\left[\omega_{\epsilon}(k)+\omega_{\epsilon}\left(\ell^{\prime}\right)\right]\right\}} \\
& \left.-\frac{\tilde{g}\left(\lambda \epsilon / 2-i\left[\epsilon \xi-\omega_{\epsilon}(k)\right]\right)}{\epsilon \lambda / 2-i\left[\epsilon \xi+\omega_{\epsilon}(k)+\omega_{\epsilon}(\ell)\right]} \cdot \frac{\tilde{g}\left(\lambda \epsilon / 2+i\left[\epsilon \xi-\omega_{\epsilon}(k)\right]\right)}{\epsilon \lambda / 2+i\left[\epsilon \xi+\omega_{\epsilon}(k)-\omega_{\epsilon}\left(\ell^{\prime}\right)\right]}\right\} .
\end{aligned}
$$

Substituting for $\operatorname{Re} \overline{\mathfrak{d}}_{\epsilon}^{2}(\lambda, k)$ from (B.36) into (B.33) we obtain $\overline{\mathfrak{L}}_{\text {scat }, 2}^{\epsilon}(\lambda)=\overline{\mathfrak{L}}_{\text {scat }, 21}^{\epsilon}(\lambda)+\overline{\mathfrak{L}}_{\text {scat }, 22}^{\epsilon}(\lambda)$, where the terms in the right hand side correspond to $R_{\epsilon}(\lambda, k), \rho_{\epsilon}(\lambda, k)$ respectively. As for $\rho_{\epsilon}(\lambda, k)$ we expect its contribution to be small in the limit and $\lim _{\epsilon \rightarrow 0+} \overline{\mathfrak{L}}_{\text {scat,22 }}^{\epsilon}(\lambda)=0$. In fact, we have.

Lemma B.7 For each $\lambda>0$ we have

$$
\lim _{\epsilon \rightarrow 0+} \int_{\mathbb{T}}\left|\rho_{\epsilon}(\lambda, k)\right| d k=0 .
$$

The proof of the lemma follows closely the argument presented in the proof Lemma 6.1 in [13], so we will not present it here.

Concerning $\overline{\mathfrak{L}}_{\text {scat,21 }}^{\epsilon}(\lambda)$, we note first that, by the same type of estimate as in (B.47),

$$
\lim _{\epsilon \rightarrow 0+}\left[\overline{\mathfrak{L}}_{\text {scat }, 21}^{\epsilon}(\lambda)-\mathfrak{L}_{\text {scat }, 21}^{\epsilon}(\lambda)\right]=0
$$

where

$$
\mathfrak{L}_{\text {scat }, 21}^{\epsilon}(\lambda):=-\frac{\gamma_{1}}{2} \int_{\mathbb{T}} R_{\epsilon}^{0}(\lambda, k) \hat{G}^{\star}(k) d k
$$

and

$$
\begin{aligned}
& R_{\epsilon}^{0}(\lambda, k):=-\frac{\gamma_{1}\left(\lambda+2 \gamma_{0} \epsilon R(k)\right)}{2^{3} \cdot \pi \epsilon^{2}} \int_{\mathbb{R}} \frac{d \xi}{\left(\lambda / 2+\gamma_{0} R(k)\right)^{2}+\xi^{2}} \int_{\mathbb{T}^{2}} d \ell d \ell^{\prime} \epsilon\left\langle\hat{\psi}(\ell) \hat{\psi}^{*}\left(\ell^{\prime}\right)\right\rangle_{\mu_{\epsilon}} \\
& \times \frac{\left|\tilde{g}_{\epsilon}(\lambda \epsilon / 2-i[\epsilon \xi+\omega(k)])\right|^{2}}{\lambda / 2-i\left\{\xi+\epsilon^{-1}[\omega(k)-\omega(\ell)]\right\}} \times \frac{1}{\lambda / 2+i\left\{\xi+\epsilon^{-1}\left[\omega(k)-\omega\left(\ell^{\prime}\right)\right]\right\}} .
\end{aligned}
$$

Using (B.37) and the change of variables

$$
\ell=: k^{\prime}+\frac{\epsilon \eta^{\prime}}{2}, \quad \ell^{\prime}=: k^{\prime}-\frac{\epsilon \eta^{\prime}}{2}
$$


we can write

$$
\begin{aligned}
& \mathfrak{L}_{\text {scat }, 21}^{\epsilon}(\lambda):=\frac{\gamma_{1}^{2}\left(\lambda+2 \gamma_{0} \epsilon R(k)\right)}{2^{3} \pi \epsilon} \int_{\mathbb{R}} d \xi \int_{\mathbb{T} \times T_{\epsilon}} \frac{\widehat{W}_{\epsilon,+}^{u n}\left(0, \eta^{\prime}, k^{\prime}\right) G^{*}(k) d k d \eta^{\prime} d k^{\prime}}{\lambda / 2-i\left\{\xi+\epsilon^{-1}\left[\omega(k)-\omega\left(k^{\prime}+\epsilon \eta^{\prime} / 2\right)\right]\right\}} \\
& \times \frac{\left|\tilde{g}_{\epsilon}(\lambda \epsilon / 2-i[\epsilon \xi+\omega(k)])\right|^{2}}{\lambda / 2+i\left\{\xi+\epsilon^{-1}\left[\omega(k)-\omega\left(k^{\prime}-\epsilon \eta^{\prime} / 2\right)\right]\right\}} \times \frac{1}{\left(\lambda / 2+\gamma_{0} R(k)\right)^{2}+\xi^{2}} .
\end{aligned}
$$

Here $T_{\epsilon}$ is the image of $\mathbb{T}_{\epsilon} \times \mathbb{T}$ under the change of variables. In fact, we may discard the contribution due to large $\eta^{\prime}$, thanks to assumption (2.52). The main contribution to the limit comes therefore from the regions where $\omega(k) \approx \omega\left(k^{\prime}\right)$, that is, where either $k \approx k^{\prime}$ - this generates the transmission term, or $k \approx-k^{\prime}$ - this is responsible for the reflection term in the limit. The conclusion of Lemma B.5 follows directly from the following result.

Lemma B.8 We have

$$
\lim _{\epsilon \rightarrow 0+} \mathfrak{L}_{\text {scat }, 21}^{\epsilon}(\lambda)=\frac{\gamma_{1}}{4} \sum_{\iota= \pm} \int_{\mathbb{T}} \frac{\hat{G}^{\star}(k) \text { g }(k) \widehat{W}_{0}(\eta, \iota k) d \eta d k}{\lambda+2 \gamma_{0} R(k)+\iota i \omega^{\prime}(k) \eta} .
$$

The proof of the lemma follows the argument presented in the proof of Lemma 6.2 in [13], so we omit it here.

\section{B.4 Proofs of auxiliary results}

\section{B.4.1 Proof of Lemma B.4}

We can write

$$
\begin{gathered}
\int_{\mathbb{T}}\left|\tilde{I}_{\epsilon}^{\iota_{1}, \iota_{2}}(\lambda, k)\right| d k=\epsilon^{2} \int_{\mathbb{T}} d k \mid \int_{\mathbb{T}} d \ell \int_{0}^{+\infty} \exp \left\{-\left\{\epsilon\left(\lambda+\gamma_{0} R(k)\right)-i \iota_{1} \omega_{\epsilon}(k)\right\} s\right\} g_{\epsilon}(d s) \\
\times \frac{r_{1, \epsilon}(k) r_{2, \epsilon}(\ell)}{\epsilon\left(\lambda+\gamma_{0} R(k)+\gamma_{0} R(\ell)\right)-i\left[\iota_{1} \omega_{\epsilon}(k)+\iota_{2} \omega_{\epsilon}(\ell)\right]}\left\langle\hat{\psi}^{\star}(k) \hat{\psi}(\ell)\right\rangle_{\mu_{\epsilon}} \mid \\
\leq \epsilon^{2} \int_{\mathbb{T}^{2}} d k d \ell\left|\tilde{g}_{\epsilon}\left(\epsilon\left(\lambda+\gamma_{0} R(k)\right)+i \iota_{1} \omega_{\epsilon}(k)\right)\right| \\
\times \frac{\left|r_{1, \epsilon}(k) r_{2, \epsilon}(\ell)\right|}{\epsilon\left(\lambda+\gamma_{0} R(k)+\gamma_{0} R(\ell)\right)-i\left[\iota_{1} \omega_{\epsilon}(k)+\iota_{2} \omega_{\epsilon}(\ell)\right] \mid}\left|\left\langle\hat{\psi}^{\star}(k) \hat{\psi}(\ell)\right\rangle_{\mu_{\epsilon}}\right| .
\end{gathered}
$$

The expression in the right hand side can be estimated by $I_{\epsilon}^{\iota_{1} \iota_{2}}$, where

$$
I_{\epsilon}^{\iota}:=\epsilon^{2} r_{1, *} r_{2, *}\left\|\tilde{g}_{\epsilon}\right\|_{\infty} \int_{\mathbb{T}^{2}} \frac{\left|\left\langle\hat{\psi}^{\star}(k) \hat{\psi}(\ell)\right\rangle_{\mu_{\epsilon}}\right| d k d \ell}{\epsilon\left(\lambda+\gamma_{0} R(k)+\gamma_{0} R(\ell)\right)+\left|\omega_{\epsilon}(k)+\iota \omega_{\epsilon}(\ell)\right|} .
$$

We need to show that

$$
I_{\epsilon}^{\iota} \preceq \epsilon^{1 / 2} \log \epsilon^{-1}, \quad \epsilon \in(0,1]
$$


for $\iota= \pm$. Note that

$$
\begin{aligned}
& \mathfrak{r}_{\epsilon}:=\sup _{A \in \mathbb{R}, k \in \mathbb{T}}\left|\frac{1}{\left|\epsilon \lambda / 2-i\left(\omega_{\epsilon}(k)-A\right)\right|}-\frac{1}{|\epsilon \lambda / 2-i(\omega(k)-A)|}\right| \\
& =\sup _{A \in \mathbb{R}, k \in \mathbb{T}} \frac{\epsilon^{2} d k}{\left|\epsilon \lambda / 2-i\left(\omega_{\epsilon}(k)-A\right)\right||\epsilon \lambda / 2-i(\omega(k)-A)|} \preceq 1, \quad \epsilon \in(0,1] .
\end{aligned}
$$

Let

$$
\tilde{I}_{\epsilon}^{\iota}:=\epsilon^{2} r_{1, *} r_{2, *}\left\|\tilde{g}_{\epsilon}\right\|_{\infty} \int_{\mathbb{T}^{2}} \frac{\left|\left\langle\hat{\psi}^{\star}(k) \hat{\psi}(\ell)\right\rangle_{\mu_{\epsilon}}\right| d k d \ell}{\epsilon\left(\lambda+\gamma_{0} R(k)+\gamma_{0} R(\ell)\right)+|\omega(k)+\iota \omega(\ell)|}, \quad \epsilon \in(0,1] .
$$

We have

$$
\left|\tilde{I}_{\epsilon}^{\iota}-I_{\epsilon}^{\iota}\right| \leq \epsilon^{2} r_{1, *} r_{2, *} \mathfrak{r}_{\epsilon}\left\|\tilde{g}_{\epsilon}\right\|_{\infty} \int_{\mathbb{T}^{2}}\left|\left\langle\hat{\psi}^{\star}(k) \hat{\psi}(\ell)\right\rangle_{\mu_{\epsilon}}\right| d k d \ell \preceq \epsilon, \quad \epsilon \in(0,1]
$$

as $\epsilon \rightarrow 0$.

In the case $\iota=+$ we can write

$$
\left.\tilde{I}_{\epsilon}^{+} \leq 2 r_{1, *} r_{2, *}\left\|\tilde{g}_{\epsilon}\right\|_{\infty}\left\{\epsilon \int_{\mathbb{T}}\left|\left\langle\hat{\psi}^{\star}(k)\right\rangle_{\mu_{\epsilon}}\right|^{2} d k\right\} \int_{\mathbb{T}} \frac{\epsilon d \ell}{\epsilon \lambda+\omega(\ell)} \preceq \epsilon \log \epsilon^{-1}, \quad \epsilon \in(), 1\right] .
$$

In the case $\iota=-$ we can write

$$
\tilde{I}_{\epsilon}^{-} \leq 2 r_{1, *} r_{2, *} \Gamma_{\epsilon}\left\|\tilde{g}_{\epsilon}\right\|_{\infty}\left\{\epsilon \int_{\mathbb{T}}\left|\left\langle\hat{\psi}^{\star}(k)\right\rangle_{\mu_{\epsilon}}\right|^{2} d k\right\}
$$

with

$$
\Gamma_{\epsilon}:=\sup _{A \in \mathbb{R}} \int_{\mathbb{T}} \frac{\epsilon d k}{\epsilon \lambda+|\omega(k)-A|}
$$

Note that $\Gamma_{\epsilon}=\Gamma_{\epsilon}^{+}+\Gamma_{\epsilon}^{-}$, with

$$
\Gamma_{\epsilon}^{ \pm}:=\sup _{A \in \mathbb{R}} \int_{\omega_{\min }}^{\omega_{\max }} \frac{\epsilon d u}{(\epsilon \lambda+|u-A|)\left|\omega^{\prime}\left(\omega_{ \pm}(u)\right)\right|} .
$$

Recall that $\omega_{-}, \omega_{+}$are the decreasing and increasing branches of the inverse function of the dispersion relation $\omega(\cdot)$. Our assumptions on the dispersion relation imply that

$$
\left|\omega^{\prime}\left(\omega_{ \pm}(u)\right)\right| \approx\left(\omega_{\max }-u\right)^{1 / 2}, \text { for } \omega_{\max }-u \ll 1
$$

The consideration near the minimum of $\omega$ is identical unless $\omega_{\min }=0$, in which case $\left|\omega^{\prime}(k)\right|$ stays uniformly positive near the minimum. Therefore, we have

$$
\Gamma_{\epsilon}^{ \pm} \preceq \sup _{A \in[0,1]} \int_{0}^{1} \frac{\epsilon d u}{(\epsilon+|u-A|) \sqrt{u}} \preceq \epsilon^{1 / 2} \log \epsilon^{-1}
$$

and we conclude that (B.46) holds. 


\section{B.4.2 Proof of Lemma B.6}

We have

$$
\begin{aligned}
& \left\|\widetilde{I I}_{\epsilon}^{\iota_{1}, \iota_{2}, \iota_{3}}(\lambda)\right\|_{L^{1}(\mathbb{T})}=\epsilon^{2} \int_{\mathbb{T}} d k \mid \int_{0}^{+\infty} \int_{0}^{+\infty} e^{-\epsilon \lambda\left(t+t^{\prime}\right) / 2} \delta\left(t-t^{\prime}\right) d t d t^{\prime} \int_{0}^{t} d s \int_{0}^{t^{\prime}} g_{\epsilon}\left(d s^{\prime}\right) \int_{0}^{s} g_{\epsilon}\left(d s_{1}\right) \int_{\mathbb{T}^{2}} d \ell d \ell^{\prime} \\
& \times r_{1, \epsilon}(k) r_{2, \epsilon}(\ell) r_{3, \epsilon}\left(\ell^{\prime}\right) e^{\lambda_{\iota_{2}}(\ell)\left(s-s_{1}\right)} e^{\lambda_{\iota_{3}}\left(\ell^{\prime}\right)\left(t^{\prime}-s^{\prime}\right)} e^{\lambda_{\iota_{1}}(k)(t-s)}\left\langle\hat{\psi}^{\star}(\ell) \hat{\psi}\left(\ell^{\prime}\right)\right\rangle_{\mu_{\epsilon}} \mid \\
& \quad=\frac{\epsilon^{2}}{2 \pi} \int_{\mathbb{T}} d k \mid \int_{\mathbb{R}} d \xi \int_{0}^{+\infty} \int_{0}^{+\infty} e^{-\epsilon \lambda\left(t+t^{\prime}\right) / 2} e^{i \xi\left(t-t^{\prime}\right)} d t d t^{\prime} \int_{0}^{t} d s \int_{0}^{t^{\prime}} g_{\epsilon}\left(d s^{\prime}\right) \int_{0}^{s} g_{\epsilon}\left(d s_{1}\right) \int_{\mathbb{T}^{2}} d \ell d \ell^{\prime} \\
& \quad \times r_{1, \epsilon}(k) r_{2, \epsilon}(\ell) r_{3, \epsilon}\left(\ell^{\prime}\right) e^{\lambda_{\iota_{2}}(\ell)\left(s-s_{1}\right)} e^{\lambda_{\iota_{3}}\left(\ell^{\prime}\right)\left(t^{\prime}-s^{\prime}\right)} e^{\lambda_{\iota_{1}}(k)(t-s)}\left\langle\hat{\psi}^{\star}(\ell) \hat{\psi}\left(\ell^{\prime}\right)\right\rangle_{\mu_{\epsilon}} \mid \cdot
\end{aligned}
$$

Note that

$$
\begin{aligned}
& \int_{0}^{+\infty} e^{-(\epsilon \lambda / 2-i \xi) t} d t \int_{0}^{t} d s \int_{0}^{s} g_{\epsilon}\left(d s_{1}\right) e^{\lambda_{\iota_{2}}(\ell)\left(s-s_{1}\right)} e^{\lambda_{\iota_{1}}(k)(t-s)} \\
& =\int_{0}^{+\infty} d s \int_{0}^{s} g_{\epsilon}\left(d s_{1}\right) \frac{e^{\lambda_{\iota_{2}}(\ell)\left(s-s_{1}\right)} \exp \{-(\epsilon \lambda / 2-i \xi) s\}}{\epsilon \lambda / 2-i \xi-\lambda_{\iota_{1}}(k)} \\
& =\frac{\tilde{g}_{\epsilon}(\epsilon \lambda / 2-i \xi)}{\left[\epsilon \lambda / 2-i \xi-\lambda_{\iota_{1}}(k)\right]\left[\epsilon \lambda / 2-i \xi-\lambda_{\iota_{2}}(\ell)\right]} .
\end{aligned}
$$

Similarly

$$
\int_{0}^{+\infty} e^{-(\epsilon \lambda / 2+i \xi) t^{\prime}} d t^{\prime} \int_{0}^{t^{\prime}} g_{\epsilon}\left(d s^{\prime}\right) e^{\lambda_{\iota_{3}}\left(\ell^{\prime}\right)\left(t^{\prime}-s^{\prime}\right)}=\frac{\tilde{g}_{\epsilon}(\epsilon \lambda / 2+i \xi)}{\epsilon \lambda / 2+i \xi-\lambda_{\iota_{3}}\left(\ell^{\prime}\right)} .
$$

Taking the above into account we obtain

$$
\begin{aligned}
& \left\|\widetilde{I I}_{\epsilon}^{\iota_{1}, \iota_{2}, \iota_{3}}(\lambda)\right\|_{L^{1}(\mathbb{T})}=\left.\frac{\epsilon^{2}}{2 \pi} \int_{\mathbb{T}} d k\left|\int_{\mathbb{R}} d \xi \int_{\mathbb{T}^{2}} d \ell d \ell^{\prime}\left\langle\hat{\psi}^{\star}(\ell) \hat{\psi}\left(\ell^{\prime}\right)\right\rangle_{\mu_{\epsilon}}\right| \tilde{g}_{\epsilon}(\epsilon \lambda / 2-i \xi)\right|^{2} \\
& \times\left\{\left[\epsilon\left(\lambda / 2+\gamma_{0} R(k)\right)-i\left(\xi+\iota_{1} \omega_{\epsilon}(k)\right)\right]\left[\epsilon\left(\lambda / 2+\gamma_{0} R(k)\right)-i\left(\xi+\iota_{2} \omega_{\epsilon}(\ell)\right)\right]\left[\epsilon\left(\lambda / 2+\gamma_{0} R(k)\right)+i\left(\xi-\iota_{3} \omega_{\epsilon}\left(\ell^{\prime}\right)\right)\right]\right\} \mid .
\end{aligned}
$$

Consider only the case $\iota_{1}=\iota_{2}=\iota_{3}=+$, as the other ones can be done in a similar fashion. We omit writing the superscripts in what follows. Change variables $\ell:=k^{\prime}-\epsilon \eta / 2, \ell^{\prime}=k^{\prime}-\epsilon \eta / 2$ and obtain

$$
\begin{aligned}
& \left\|\widetilde{I I}_{\epsilon}(\lambda)\right\|_{L^{1}(\mathbb{T})}=\left.\frac{\epsilon^{2}}{\pi} \int_{\mathbb{T}} d k\left|\int_{\mathbb{R}} d \xi \int_{T_{\epsilon}} d \eta d k^{\prime} \widehat{W}_{\epsilon}\left(\eta, k^{\prime}\right)\right| \tilde{g}_{\epsilon}(\epsilon \lambda / 2-i \xi)\right|^{2}\left\{\left[\epsilon\left(\lambda / 2+\gamma_{0} R(k)\right)-i\left(\xi+\omega_{\epsilon}(k)\right)\right]\right. \\
& \left.\left[\epsilon\left(\lambda / 2+\gamma_{0} R(k)\right)-i\left(\xi+\omega_{\epsilon}\left(k^{\prime}-\epsilon \eta / 2\right)\right)\right]\left[\epsilon\left(\lambda / 2+\gamma_{0} R(k)\right)+i\left(\xi-\omega_{\epsilon}\left(k^{\prime}+\epsilon \eta / 2\right)\right)\right]\right\}^{-1} \mid
\end{aligned}
$$

Thanks to (2.51) and (3.18) we can estimate

$$
\begin{aligned}
& \left\|\widetilde{I I}_{\epsilon}(\lambda)\right\|_{L^{1}(\mathbb{T})} \preceq \epsilon^{2} \int_{\mathbb{R}} d \xi \int_{\mathbb{R}} \varphi(\eta) d \eta \int_{\mathbb{T}^{2}} \mid\left[\epsilon\left(\lambda / 2+\gamma_{0} R(k)\right)-i\left(\xi+\omega_{\epsilon}(k)\right)\right] \\
& \times\left.\left[\epsilon\left(\lambda / 2+\gamma_{0} R(k)\right)-i\left(\xi+\omega_{\epsilon}\left(k^{\prime}-\epsilon \eta / 2\right)\right)\right]\left[\epsilon\left(\lambda / 2+\gamma_{0} R(k)\right)+i\left(\xi-\omega_{\epsilon}\left(k^{\prime}+\epsilon \eta / 2\right)\right)\right]\right|^{-1} d k d k^{\prime}, \quad \epsilon \in(0,1] .
\end{aligned}
$$


We need to prove that the right hand side vanishes, as $\epsilon \rightarrow 0$. Similarly to what has been done in the proof of Lemma B.4, it suffices only to show that

$$
\lim _{\epsilon \rightarrow 0} J_{\epsilon}=0
$$

where

$$
\begin{aligned}
& J_{\epsilon}:=\epsilon^{2} \int_{\mathbb{R}} d \xi \int_{\mathbb{R}} \varphi(\eta) d \eta \int_{\mathbb{T}^{2}} \mid[\epsilon \lambda / 2-i(\xi+\omega(k))] \\
& \times\left.\left[\epsilon \lambda / 2-i\left(\xi+\omega\left(k^{\prime}-\epsilon \eta / 2\right)\right)\right]\left[\epsilon \lambda / 2+i\left(\xi-\omega\left(k^{\prime}+\epsilon \eta / 2\right)\right)\right]\right|^{-1} d k d k^{\prime}
\end{aligned}
$$

Changing variables $\xi+\omega(k):=\epsilon \xi^{\prime}$ we conclude that

$$
\begin{aligned}
& J_{\epsilon} \approx \int_{\mathbb{R}} d \xi \int_{\mathbb{R}} \varphi(\eta) d \eta \int_{\mathbb{T}^{2}}\left\{(1+|\xi|)\left(1+\left|\xi+\epsilon^{-1}\left(\omega\left(k^{\prime}-\epsilon \eta / 2\right)-\omega(k)\right)\right|\right)\right. \\
& \left.\left(1+\mid \xi+\epsilon^{-1}\left(\omega(k)-\omega\left(k^{\prime}+\epsilon \eta / 2\right)\right)\right)\right\}^{-1} d k d k^{\prime} \leq J_{\epsilon}^{1}+J_{\epsilon}^{2}
\end{aligned}
$$

with

$$
J_{\epsilon}^{1}:=\frac{1}{2} \int_{\mathbb{R}} d \xi \int_{\mathbb{R}} \varphi(\eta) d \eta \int_{\mathbb{T}^{2}}\left\{(1+|\xi|)\left(1+\left|\xi+\epsilon^{-1}\left(\omega\left(k^{\prime}-\epsilon \eta / 2\right)-\omega(k)\right)\right|\right)^{2}\right\}^{-1} d k d k^{\prime}
$$

and

$$
J_{\epsilon}^{2}:=\frac{1}{2} \int_{\mathbb{R}} d \xi \int_{\mathbb{R}} \varphi(\eta) d \eta \int_{\mathbb{T}^{2}}\left\{(1+|\xi|)\left(1+\mid \xi+\epsilon^{-1}\left(\omega(k)-\omega\left(k^{\prime}+\epsilon \eta / 2\right)\right)\right)^{2}\right\}^{-1} d k d k^{\prime}
$$

Using an elementary estimate

$$
\int_{\mathbb{R}} \frac{1}{1+|x+a|} \times \frac{d x}{1+x^{2}} \preceq \frac{1}{1+|a|}, \quad a \in \mathbb{R}
$$

we conclude that

$$
J_{\epsilon}^{1} \preceq \frac{1}{2} \int_{\mathbb{R}} \varphi(\eta) d \eta \int_{\mathbb{T}^{2}}\left\{1+\left|\epsilon^{-1}\left(\omega\left(k^{\prime}-\epsilon \eta / 2\right)-\omega(k)\right)\right|\right\}^{-1} d k d k^{\prime} \rightarrow 0,
$$

by virtue of the dominated convergence theorem. Estimates for $J_{\epsilon}^{2}$ are similar.

\section{B.4.3 Proof of formula $(\overline{B .36})$}

From (B.35) we have

$$
\begin{aligned}
& 2 \operatorname{Re} \overline{\mathfrak{d}}_{\epsilon}^{2}(\lambda, k)=-\gamma_{1} \epsilon\left\langle\int _ { 0 } ^ { + \infty } e ^ { - \lambda \epsilon t } e ^ { - 2 \gamma _ { 0 } \epsilon R ( k ) t } \frac { d } { d t } \left\{\left[\int_{0}^{t} e^{\gamma_{0} \epsilon R(k) t s} \cos \left(\omega_{\epsilon}(k) s\right) g_{\epsilon} * \bar{p}_{0}^{0}(s) d s\right]^{2}\right.\right. \\
& \left.\left.+\left[\int_{0}^{t} e^{\gamma_{0} \epsilon R(k) t s} \sin (\omega(k) s) g_{\epsilon} * \bar{p}_{0}^{0}(s) d s\right]^{2}\right\} d t\right\rangle_{\mu_{\epsilon}} .
\end{aligned}
$$


Integrating by parts, we obtain

$$
2 \operatorname{Re} \mathfrak{d}_{\epsilon}^{2}(\lambda, k)=C_{\epsilon}(\lambda, k)+S_{\epsilon}(\lambda, k) .
$$

The first term in the right side is

$$
C_{\epsilon}(\lambda, k)=-\frac{\gamma_{1}}{4 \pi} \epsilon\left(\lambda+2 \gamma_{0} \epsilon R(k)\right) \int_{\mathbb{R}} d \xi \int_{\mathbb{T}^{2}} \epsilon\left\langle\hat{\psi}(\ell) \hat{\psi}^{*}\left(\ell^{\prime}\right)\right\rangle_{\mu_{\epsilon}} \Xi_{\epsilon}(\ell, k, \lambda, \xi) \Xi_{\epsilon}^{\star}\left(\ell^{\prime}, k, \lambda, \xi\right) d \ell d \ell^{\prime},
$$

with

$$
\Xi_{\epsilon}(\ell, k, \lambda, \xi):=\int_{0}^{+\infty} e^{\gamma_{0} \epsilon R(k) s} \cos \left(\omega_{\epsilon}(k) s\right) d s\left\{\int_{0}^{s} e^{-i \omega_{\epsilon}(\ell)(s-\tau)} g_{\epsilon}(d \tau) \int_{s}^{+\infty} e^{-\left[\left(\lambda / 2+\gamma_{0} R(k)\right) \epsilon-i \xi\right] t} d t\right\} .
$$

Integrating out first the $t$ variable, and then the $s$ varable, we obtain

$$
\Xi_{\epsilon}(\ell, k, \lambda, \xi)=\frac{1}{2\left[\left(\lambda / 2+\gamma_{0} R(k)\right) \epsilon-i \xi\right]}\left\{\frac{\tilde{g}_{\epsilon}\left(\lambda \epsilon / 2-i\left[\xi+\omega_{\epsilon}(k)\right]\right)}{\lambda \epsilon / 2-i\left(\xi+\omega_{\epsilon}(k)-\omega_{\epsilon}(\ell)\right)}+\frac{\left.\tilde{g}_{\epsilon}\left(\lambda \epsilon / 2-i\left[\xi-\omega_{\epsilon}(k)\right)\right]\right)}{\lambda \epsilon / 2-i\left[\xi+\omega_{\epsilon}(k)+\omega_{\epsilon}(\ell)\right]}\right\} .
$$

Hence, after a change of variables $\xi:=\epsilon \xi^{\prime}$, we get

$$
\begin{aligned}
& C_{\epsilon}(\lambda, k)=-\frac{\gamma_{1}\left(\lambda+2 \gamma_{0} \epsilon R(k)\right)}{2^{4} \cdot \pi \epsilon^{2}} \int_{\mathbb{R}} \frac{d \xi}{\left(\lambda / 2+\gamma_{0} R(k)\right)^{2}+\xi^{2}} \int_{\mathbb{T}^{2}} d \ell d \ell^{\prime} \epsilon\left\langle\hat{\psi}(\ell) \hat{\psi}^{*}\left(\ell^{\prime}\right)\right\rangle_{\mu_{\epsilon}} \\
& \times\left\{\frac{\tilde{g}_{\epsilon}\left(\lambda \epsilon / 2-i\left[\epsilon \xi+\omega_{\epsilon}(k)\right]\right)}{\lambda / 2-i\left\{\xi+\epsilon^{-1}\left[\omega_{\epsilon}(k)-\omega_{\epsilon}(\ell)\right]\right\}}+\frac{\tilde{g}_{\epsilon}\left(\lambda \epsilon / 2-i\left[\epsilon \xi-\omega_{\epsilon}(k)\right]\right)}{\lambda / 2-i\left\{\xi+\epsilon^{-1}\left[\omega_{\epsilon}(k)+\omega_{\epsilon}(\ell)\right]\right\}}\right\} \\
& \times\left\{\frac{\tilde{g}_{\epsilon}\left(\lambda \epsilon / 2+i\left[\epsilon \xi+\omega_{\epsilon}(k)\right]\right)}{\lambda / 2+i\left\{\xi+\epsilon^{-1}\left[\omega_{\epsilon}(k)-\omega_{\epsilon}\left(\ell^{\prime}\right)\right]\right\}}+\frac{\left.\tilde{g}\left(\lambda \epsilon / 2+i\left[\epsilon \xi+\omega_{\epsilon}(k)\right]\right)\right)}{\lambda / 2+i\left\{\xi+\epsilon^{-1}\left[\omega_{\epsilon}(k)+\omega_{\epsilon}\left(\ell^{\prime}\right)\right]\right\}}\right\} .
\end{aligned}
$$

A similar calculation leads to

$$
\begin{aligned}
& S_{\epsilon}(\lambda, k)=\frac{\gamma_{1}\left(\lambda+2 \gamma_{0} \epsilon R(k)\right)}{2^{4} \pi \epsilon^{2}} \int_{\mathbb{R}} \frac{d \xi}{\left(\lambda / 2+\gamma_{0} R(k)\right)^{2}+\xi^{2}} \int_{\mathbb{T}^{2}} d \ell d \ell^{\prime} \epsilon\left\langle\hat{\psi}(\ell) \hat{\psi}^{*}\left(\ell^{\prime}\right)\right\rangle_{\mu_{\epsilon}} \\
& \times\left\{\frac{\tilde{g}_{\epsilon}\left(\lambda \epsilon / 2-i\left[\epsilon \xi+\omega_{\epsilon}(k)\right]\right)}{\lambda / 2-i\left\{\xi+\epsilon^{-1}\left[\omega_{\epsilon}(k)-\omega_{\epsilon}(\ell)\right]\right\}}-\frac{\tilde{g}_{\epsilon}\left(\lambda \epsilon / 2-i\left[\epsilon \xi-\omega_{\epsilon}(k)\right]\right)}{\lambda / 2-i\left\{\xi+\epsilon^{-1}\left[\omega_{\epsilon}(k)+\omega_{\epsilon}(\ell)\right]\right\}}\right\} \\
& \times\left\{\frac{\left.\tilde{g}_{\epsilon}\left(\lambda \epsilon / 2+i\left(\epsilon \xi-\omega_{\epsilon}(k)\right]\right)\right)}{\lambda / 2+i\left\{\xi-\epsilon^{-1}\left[\omega_{\epsilon}(k)+\omega_{\epsilon}\left(\ell^{\prime}\right)\right]\right\}}-\frac{\tilde{g}_{\epsilon}\left(\lambda \epsilon / 2+i\left[\epsilon \xi+\omega_{\epsilon}(k)\right]\right)}{\lambda / 2+i\left\{\xi+\epsilon^{-1}\left[\omega_{\epsilon}(k)-\omega_{\epsilon}\left(\ell^{\prime}\right)\right]\right\}}\right\} .
\end{aligned}
$$

Putting (B.53) $-(\underline{B .56})$ together, gives (B.36).

\section{Proof of Proposition 3.1}

Let $(\mathrm{cf}(3.13))$

$$
\tilde{j}_{\epsilon}(\lambda, k):=\int_{0}^{+\infty} e^{-\lambda t} j_{\epsilon}(t, k) d t, \quad \operatorname{Re} \lambda>0 .
$$

From (C.1) and (3.13) it follows that

$$
\tilde{j}_{\epsilon}(\lambda, k)=\frac{\lambda \sqrt{1-(\epsilon \beta)^{2}}}{2\left(\lambda+\epsilon \gamma_{0} R(k)\right)}\left\{\frac{1}{\lambda+\epsilon \gamma_{0} R(k)+i \omega_{\epsilon}(k)}+\frac{1}{\lambda+\epsilon \gamma_{0} R(k)-i \omega_{\epsilon}(k)}\right\} .
$$


Also, since $\Omega_{0}(k)-\Omega_{\epsilon}(k)=\epsilon \gamma_{0} R(k) \mathfrak{f} \otimes \mathfrak{g}($ see (3.4) $)$ we have

$$
\begin{gathered}
\tilde{j}_{0}(\lambda, k)-\tilde{j}_{\epsilon}(\lambda, k)=\frac{1}{2}\left[\left(\lambda-\Omega_{0}(k)\right)^{-1}-\left(\lambda-\Omega_{\epsilon}(k)\right)^{-1}\right] \mathfrak{f} \cdot \mathfrak{f} \\
=\frac{1}{2}\left(\lambda-\Omega_{0}(k)\right)^{-1}\left(\Omega_{0}(k)-\Omega_{\epsilon}(k)\right)\left(\lambda-\Omega_{\epsilon}(k)\right)^{-1} \mathfrak{f} \cdot \mathfrak{f}=\frac{1}{2} \epsilon \gamma_{0} R(k) \tilde{j}_{\epsilon}(\lambda, k) \tilde{j}_{0}(\lambda, k) .
\end{gathered}
$$

The following result holds.

Proposition C.1 We have

$$
\tilde{J}(\lambda)=\tilde{J}_{\epsilon}(\lambda)+\epsilon \gamma_{0} \tilde{\mathcal{R}}_{\epsilon}(\lambda), \quad \epsilon \in(0,1], \lambda \in \mathbb{C}_{+},
$$

where

$$
\tilde{\mathcal{R}}_{\epsilon}(\lambda)=\int_{\mathbb{T}} R(k) \tilde{j}_{\epsilon}(\lambda, k) \tilde{j}_{0}(\lambda, k) d k, \quad \lambda \in \mathbb{C}_{+} .
$$

In addition, for any $p \in(1,2)$ and a uniformly continuous and bounded function $\xi: \mathbb{R} \rightarrow \mathbb{R}_{+}$ satisfying

$$
\xi(\eta) \geq \xi_{0}, \quad \eta \in \mathbb{R}
$$

we have

$$
\lim _{\epsilon \rightarrow 0} \epsilon^{p} \int_{\mathbb{R}}\left|\tilde{\mathcal{R}}_{\epsilon}(\epsilon \xi(\eta)+i \eta)\right|^{p} d \eta=0
$$

Proof. Identity (C.4) follows directly from (C.3). Letting $\lambda:=\epsilon \xi(\eta)+i \eta$, using (C.2) together with (C.5) and the change of variables $k \mapsto v=\omega(k)$ we can write

$$
\tilde{J}(\epsilon \xi(\eta)+i \eta)-\tilde{J}_{\epsilon}(\epsilon \xi(\eta)+i \eta)=\frac{1}{2} \sum_{\iota= \pm}\left\{\int_{\mathbb{R}} \frac{\chi_{\iota, \epsilon}(v) d v}{\epsilon \xi(\eta)+i(\eta+v)}+\int_{\mathbb{R}} \frac{\chi_{\iota, \epsilon}(v) d v}{\epsilon \xi(\eta)+i(\eta-v)}\right\},
$$

with

$$
\chi_{\iota, \epsilon}(v)=\iota \epsilon \gamma_{0} R\left(\omega_{+}(v)\right) \tilde{j}_{\epsilon}\left(\epsilon \xi(\eta)+i \eta, \omega_{+}(v)\right) \frac{1_{\left[\omega_{\min }, \omega_{\max }\right]}(v)}{\omega^{\prime}\left(\omega_{+}(v)\right)} .
$$

Here, (see Section 2.3.1) $\omega_{ \pm}$are the two branches of inverses of the unimodal dispersion relation $\omega$, with $\omega_{+}:\left[\omega_{\min }, \omega_{\max }\right] \rightarrow[0,1 / 2]$ and $\omega_{-}:=-\omega_{+}$. In addition to (B.49), in the optical case, we also have

$$
\left|\omega^{\prime}\left(\omega_{ \pm}(u)\right)\right| \approx\left(u-\omega_{\min }\right)^{1 / 2}, \text { for } u-\omega_{\min } \ll 1
$$

Hence,

$$
\begin{aligned}
& \epsilon \gamma_{0} R(k)\left|\tilde{j}_{\epsilon}(\epsilon \xi(\eta)+i \eta, k)\right| \\
& \leq \frac{1}{2}\left\{\left|\frac{\epsilon \gamma_{0} R(k)}{\epsilon\left(\xi(\eta)+\gamma_{0} R(k)\right)+i\left(\eta+\omega_{\epsilon}(k)\right)}\right|+\left|\frac{\epsilon \gamma_{0} R(k)}{\epsilon\left(\xi(\eta)+\gamma_{0} R(k)\right)+i\left(\eta-\omega_{\epsilon}(k)\right)}\right|\right\} .
\end{aligned}
$$


The above allows us to conclude that

$$
\epsilon \gamma_{0} R(k)\left|\tilde{j}_{\epsilon}(\epsilon \xi(\eta)+i \eta, k)\right| \leq C
$$

for some $C>0$ independent of $\eta, \epsilon, k$ and, as a result,

$$
\left|\chi_{\iota, \epsilon}(v)\right| \leq \frac{C 1_{\left[\omega_{\min }, \omega_{\max }\right]}(v)}{\omega^{\prime}\left(\omega_{+}(v)\right)}
$$

Thus

$$
\lim _{\epsilon \rightarrow 0+} \chi_{\iota, \epsilon}(v)=0
$$

both a.s. and in the $L^{p}$ sense for any $p \in[1,2)$, see (B.49) and (C.9). Let $\xi_{0}$ be as in (C.6). Note that for any $\eta \in \mathbb{R}$

$$
\begin{aligned}
& \left|\int_{\mathbb{R}} \frac{\chi_{\iota, \epsilon}(v-\eta) d v}{\epsilon \xi(\eta)+i v}\right| \leq\left|\int_{\left[|v| \geq \epsilon \xi_{0}\right]} \frac{\chi_{\iota, \epsilon}(v-\eta) d v}{i v}\right| \\
& +\left|\int_{\left[|v| \geq \epsilon \xi_{0}\right]} \frac{\chi_{\iota, \epsilon}(v-\eta) d v}{\epsilon \xi(\eta)+i v}-\int_{\left[|v| \geq \epsilon \xi_{0}\right]} \frac{\chi_{\iota, \epsilon}(v-\eta) d v}{i v}\right|+\left|\int_{\left[|v|<\epsilon \xi_{0}\right]} \frac{\chi_{\iota, \epsilon}(v-\eta) d v}{\epsilon \xi(\eta)+i v}\right|
\end{aligned}
$$

Denote the expressions in the utmost right hand side by $\mathcal{J}_{1, \epsilon}(\eta), \mathcal{J}_{2, \epsilon}(\eta)$ and $\mathcal{J}_{3, \epsilon}(\eta)$, respectively.

Using Theorem 3.2, p. 35 of 22 ] we conclude that for any $p \in(1,+\infty)$ there exists a constant $C>0$, independent of $\epsilon>0$ such that

$$
\left\|\mathcal{J}_{1, \epsilon}\right\|_{L^{p}(\mathbb{R})} \leq C\left\|\chi_{\iota, \epsilon}\right\|_{L^{p}(\mathbb{R})}
$$

Considering $\mathcal{J}_{3, \epsilon}$, there exists $C>0$ such that

$$
\frac{1_{\left[|v|<\epsilon \xi_{0}\right]}}{|\epsilon \xi(\epsilon)+i v|} \leq \frac{C 1_{\left[|v|<\epsilon \xi_{0}\right]}}{\epsilon}
$$

thus, again (using Young's inequality) for any $p \in(1,+\infty)$ there exists $C>0$ such that

$$
\left\|\mathcal{J}_{3, \epsilon}\right\|_{L^{p}(\mathbb{R})} \leq C\left\|\chi_{\iota, \epsilon}\right\|_{L^{p}(\mathbb{R})}, \quad \epsilon \in(0,1]
$$

For $\rho \in(0,1)$ we can write

$$
\left|\mathcal{J}_{2, \epsilon}(\eta)\right| \leq \int_{\left[|v| \geq \epsilon \xi_{0}\right]} \frac{\epsilon \xi(\eta)\left|\chi_{\iota, \epsilon}(v-\eta)\right| d v}{\left[(\epsilon \xi(\eta))^{2}+v^{2}\right]^{1 / 2}|v|} \leq\|\xi\|_{\infty} \int_{\left[|v| \geq \epsilon \xi_{0}\right]} \frac{\epsilon^{\rho}\left|\chi_{\iota, \epsilon}(v-\eta)\right| d v}{|v|^{1+\rho}} .
$$

By an application of Young's inequality for convolutions we have

$$
\left\|\mathcal{J}_{2, \epsilon}\right\|_{L^{p}(\mathbb{R})} \leq 2 \epsilon^{\rho}\|\xi\|_{\infty}\left\|\chi_{\iota, \epsilon}\right\|_{L^{p}(\mathbb{R})} \int_{\epsilon \xi_{0}}^{+\infty} \frac{d v}{v^{1+\rho}} \leq C\left\|\chi_{\iota, \epsilon}\right\|_{L^{p}(\mathbb{R})}, \quad \epsilon \in(0,1],
$$

with constant $C>0$ independent of $\epsilon$. 
Taking into account all the above we conclude that

$$
\lim _{\epsilon \rightarrow 0+} \int_{\mathbb{R}}\left|\tilde{J}(\epsilon \xi(\eta)+i \eta)-\tilde{J}_{\epsilon}(\epsilon \xi(\eta)+i \eta)\right|^{p} d \eta=0
$$

for any $p \in(1,2)$.

Proposition 3.1 follows from the following result.

Proposition C.2 Suppose that $\xi: \mathbb{R} \rightarrow \mathbb{R}_{+}$is a uniformly continuous and bounded function satisfying

$$
\inf _{\eta \in \mathbb{R}} \xi(\eta)>0
$$

and $\tilde{r}_{\epsilon}(\lambda)$ is given by (3.21). Then, for any $p \in(1,+\infty)$, we have

$$
\lim _{\epsilon \rightarrow 0+} \epsilon^{p} \int_{\mathbb{R}}\left|\tilde{r}_{\epsilon}(\epsilon \xi(\eta)+i \eta)\right|^{p} d \eta=0
$$

Indeed, assume the above result and let $K$ be as in the statement of Proposition 3.1. Define $\xi_{ \pm}(\eta):=$

$K\left(\omega_{ \pm}(\eta)\right), \eta \in \overline{\mathbb{T}}_{ \pm}$and $\xi_{ \pm}(\eta)=1$, elsewhere. We conclude that in particular $\epsilon \mid \tilde{r}_{\epsilon}\left(\epsilon \xi_{ \pm}(\eta)+i \eta\right)$ converges to 0 in the Lebesgue measure on $\left[\omega_{\min }, \omega_{\max }\right]$. This obviously implies that $\epsilon \mid \tilde{r}_{\epsilon}(\epsilon K(k)-$ $i \omega(k))$ convergence in the Lebesgue measure on $\mathbb{T}$ to 0 . The $L^{p}$ convergence follows from the fact that the functions are bounded.

\section{Proof of Proposition C.2}

We have (cf (C.5D)

$$
\tilde{g}_{\epsilon}(\lambda)-\tilde{g}(\lambda)=\epsilon \gamma_{0} \gamma_{1}\left(1+\gamma_{1} \tilde{J}_{\epsilon}(\lambda)\right)^{-1}\left(1+\gamma_{1} \tilde{J}(\lambda)\right)^{-1} \tilde{\mathcal{R}}_{\epsilon}(\lambda)
$$

From the above identity we conclude that

$$
\left|\tilde{g}_{\epsilon}(\epsilon \xi(\eta)+i \eta)-\tilde{g}(\epsilon \xi(\eta)+i \eta)\right| \leq \epsilon \gamma_{0} \gamma_{1}\left|\tilde{\mathcal{R}}_{\epsilon}(\epsilon \xi(\eta)+i \eta)\right|
$$

From (C.7) we conclude that

$$
\lim _{\epsilon \rightarrow 0+} \int_{\mathbb{R}}\left|\tilde{g}_{\epsilon}(\epsilon \xi(\eta)+i \eta)-\tilde{g}(\epsilon \xi(\eta)+i \eta)\right|^{p} d \eta=0
$$

for any $p \in(1,2)$. On the other hand, thanks to (3.18), for $p \geq 2$ we get

$$
\int_{\mathbb{R}}\left|\tilde{g}_{\epsilon}(\epsilon \xi(\eta)+i \eta)-\tilde{g}(\epsilon \xi(\eta)+i \eta)\right|^{p} d \eta \leq 2^{p-3 / 2} \int_{\mathbb{R}}\left|\tilde{g}_{\epsilon}(\epsilon \xi(\eta)+i \eta)-\tilde{g}(\epsilon \xi(\eta)+i \eta)\right|^{3 / 2} d \eta \rightarrow 0
$$

as $\epsilon \rightarrow 0+$. 


\section{References}

[1] G. Bal, G. Papanicolaou and L. Ryzhik, Radiative transport limit for the random Schrödinger equation, Nonlinearity, 15, 2002, 513-529.

[2] G.Bal, G. Papanicolaou and L. Ryzhik, Self-averaging in time reversal for the parabolic wave equation, Stochastics and Dynamics, 2, 2002, 507-531.

[3] G. Bal, T. Komorowski, L. Ryzhik, Kinetic limits for waves in a random medium Kinetic and Related Models 3, (2010), pp. 529 - 644, doi:10.3934/krm.2010.3.529

[4] G. Basile, S. Olla, H. Spohn Wigner functions and stochastically perturbed lattice dynamics, Arch.Rat.Mech., Vol. 195, no. 1, 171-203, 2009.

[5] G. Basile, C. Bernardin, M. Jara, T. Komorowski and S. Olla Thermal conductivity in harmonic lattices with random collisions. in Thermal transport in low dimensions. From Statistical Physics to Nanoscale Heat Transfer., S. Lepri (edt), Lecture Notes in Physics vol. 921 (Springer 2016), pp 215-236.

[6] G. Basile, T. Komorowski, S. Olla, Diffusion limit for a kinetic equation with a thermostatted interface. Kinetic \& Related Models 2019, 12(5): 1185-1196 doi: 10.3934/krm.2019045

[7] Benamou, J.-D.; Castella, François; Katsaounis, T.; Perthame, B., High frequency limit of the Helmholtz equations. Rev. Mat. Iber. 18 (2002), 187-209.

[8] M. J. Butz, Kinetic limit for Wave Propagation in a Continuous, Weakly Random Medium Self-averaging and Convergence to a Linear Boltzmann Equation Ph. D. Dissertation, Technische Universität München, Fakultät für Mathematik Lehrstuhl für Mathematische Physik

[9] Chen,T., Convergence in higher mean of a random Schrödinger to a linear Boltzmann evolution. Commun. Math. Phys. 267, 355-392, 2006.

[10] Diestel, J.; Uhl, J. J., Jr. Vector measures. Mathematical Surveys, No. 15. American Mathematical Society, Providence, R.I., 1977. xiii+322 pp.

[11] L. Erdös and H. T. Yau, Linear Boltzmann equation as the weak coupling limit of a random Schrödinger Equation, Comm. Pure Appl. Math., 53, 2000, 667-735.

[12] A. C. Fannjiang, Self-Averaging Scaling Limits for Random Parabolic Waves. Arch. Rational Mech. Anal. 175 (2005) 343-387.

[13] T. Komorowski, S. Olla, L. Ryzhik, H. Spohn, High frequency limit for a chain of harmonic oscillators with a point Langevin thermostat, Arch. Rational Mech. An., 237, 497-543 (2020). https://doi.org/10.1007/s00205-020-01513-7.

[14] T. Komorowski, S. Olla, L. Ryzhik, Fractional diffusion limit for a kinetic equation with an interface, 2019, arxiv.org/1905.10586, to appear in Ann. Prob. 
[15] T. Komorowski, Ł. Stȩpień, Kinetic limit for a harmonic chain with a conservative Ornstein-Uhlenbeck stochastic perturbation. Kinetic \& Related Models, 11, 239-278, (2018) doi.org/10.3934/krm.2018013

[16] P. Koosis, Introduction to $H^{p}$ spaces. Cambridge Univ. Press (1980).

[17] Miller, L. Short waves through thin interfaces and 2-microlocal measures. Journ. Equat. aux Deriv. Partielles" (1997), article no. 12.

[18] Miller, L. Réfraction d óndes semi-classiques par des interfaces franches. C. R. Acad. Sci. Paris Ser. I Math. 325 (1997), no. 4, 371-376.

[19] Miller, L. Refraction of high-frequency waves density by sharp interfaces and semiclassical measures at the boundary. J. Math. Pures Appl. (9) 79 (2000), no. 3, 227-269.

[20] H. Spohn, Derivation of the transport equation for electrons moving through random impurities, Jour. Stat. Phys., 17, 1977, 385-412.

[21] Spohn, H., The phonon Boltzmann equation, properties and link to weakly anharmonic lattice dynamics, J. Stat. Phys. 124, no.2-4, 1041-1104 (2006).

[22] Stein, E., Singular Integrals and Differentiability Properties of Functions, Princeton, 1970. 